\title{
A note on the geometry and topology of almost even-Clifford Hermitian manifolds
}

\author{
Gerardo Arizmendi; Ana L. Garcia-Pulido* and Rafael Herrera*†
}

\begin{abstract}
We compute the structure groups of almost even-Clifford Hermitian manifolds and determine when such groups lead to Spin structures.
\end{abstract}

\section{Introduction}

Almost even-Clifford Hermitian structures on oriented Riemannian manifolds were introduced recently in their current form in [10] under the simpler name of even Clifford structures. They are a subject of current interest [1, 3, 5, 9, 12, although similar types of structures have been studied in the past [4, 7, 11. They are generalizations of almost Hermitian and almost quaternion-Hermitian structures, and there has been quite some interest in them. The existence of such a structure on a manifold implies the reduction of its structure group to the normalizer of the homomorphic image of a Spin group. In this paper, we identify such structure group (cf. Theorem [3.2) by using the results about their Lie algebras given in [2]. In the case of $4 m$-dimensional almost quaternion-Hermitian manifolds, we know that such manifolds are Spin when $m$ is even. This is due to the (topological) reduction of the structure group from $S O(4 n)$ to the Lie group $S p(n) S p(1)$ which, in turn, embeds into $\operatorname{Spin}(4 m)$ when $m$ is even. Thus, by analogy, we were led to study when such manifolds admit Spin structures.

Recall that an oriented $n$-dimensional Riemannian manifold is Spin if its orthonormal frame bundle $P_{S O}$ admits a double cover by a principal $\operatorname{Spin}(n)$ bundle $P_{S p i n}$

$$
\Lambda: P_{\text {Spin }} \longrightarrow P_{S O}
$$

which is $\operatorname{Spin}(n)$ equivariant, i.e. $\Lambda(p g)=\Lambda(p) \lambda_{n}(g)$ for all $g \in \operatorname{Spin}(n)$. A Riemannian manifold will automatically be Spin if its structure group reduces to a proper subgroup $G \subset S O(n)$ such that there exists a lifting map which makes the following diagram commute

$$
\begin{array}{rll} 
& & \operatorname{Spin}(n) \\
G & \hookrightarrow & \downarrow \\
& & S O(n) .
\end{array}
$$

Indeed, such a lift exists if and only if $\pi_{1}(G)$ maps trivially into $\pi_{1}(S O(n))$.

In this paper, we determine when there exists a lifting map which makes the following diagram commute (cf. Theorem 4.1)

$$
\begin{aligned}
& \operatorname{Spin}(N) \\
& \downarrow \\
\mathcal{N}_{S O(N)}(S) \hookrightarrow & S O(N) .
\end{aligned}
$$

\footnotetext{
*Centro de Investigación en Matemáticas, A. P. 402, Guanajuato, Gto., C.P. 36000, México. E-mail: gerardo@cimat.mx, lucia@cimat.mx, rherrera@cimat.mx

${ }^{\dagger}$ Partially supported by a CONACYT grant
} 
where $N$ stands for the dimension of an almost even-Clifford Hermitian manifold, $S$ denotes the homomorphic image of the aforementioned Spin group determined by the even-Clifford structure, and $\mathcal{N}_{S O(N)}(S)$ denotes its normalizer in $S O(N)$. In fact, we will verify that there is a lift for the connected component of the identity $\mathcal{N}_{S O(N)}^{0}(S)$, since the other components are diffeomorphic to it and will also lift to the Spin group. Furthermore, note that an almost even-Clifford Hermitian manifold might still be Spin even if there is no such a lifting map, as in the case of quaternionic projective spaces $\mathbb{H}^{m}$ of odd quaternionic dimension $m$. It would be interesting, at least for the authors, to find and study non-Spin almost even-Clifford manifolds of rank 4,6 and 8 .

The note is organized as follows. In Section 2, we recall some preliminaries on Clifford algebras, the Spin group and representations, almost even-Clifford manifolds, etc. In Section 3, we determine the complexifications of real representations of even Clifford algebras containing no trivial summands (cf. Theorem 3.1), identify the subgroups $\mathcal{N}_{S O(N)}^{0}(S)$ as finite quotients of products of classical groups (or real lines in some cases) and spin groups (cf. Theorem 3.2), and calculate their fundamental groups giving explicit generators (cf. Theorem 3.3). In Section 4, we determine when the aformentioned lifts exist (cf. Theorem 4.1).

Acknowledgements. The third author wishes to thank the International Centre for Theoretical Physics and the Institut des Hautes Études Scientifiques for their hospitality and support.

\section{Preliminaries}

The material presented in this section can be consulted in [6].

\subsection{Clifford algebra, spin group and representation}

Let $C l_{n}$ denote the $2^{n}$-dimensional real Clifford algebra generated by the orthonormal vectors $e_{1}, e_{2}, \ldots, e_{n} \in$ $\mathbb{R}^{n}$ subject to the relations

$$
e_{i} e_{j}+e_{j} e_{i}=-2 \delta_{i j},
$$

and $\mathbb{C} l_{n}=C l_{n} \otimes_{\mathbb{R}} \mathbb{C}$ its complexification. The even Clifford subalgebra $C l_{r}^{0}$ is defined as the invariant $(+1)$-subspace of the involution of $C l_{r}$ induced by the map $-\operatorname{Id}_{\mathbb{R}^{r}}$. For any vector $Y=y_{1} e_{1}+\cdots+y_{n} e_{n}$, the product

$$
e_{i} Y e_{i}=y_{1} e_{1}+\cdots+y_{i-1} e_{i-1}-y_{i} e_{i}+y_{i+1} e_{i+1}+\cdots+y_{n} e_{n}
$$

gives the reflection of the $i$-th coordinate, and the conjugation with the volume element $\operatorname{vol}_{n}=e_{1} \cdots e_{n}$ gives the reflection on the origin of $\mathbb{R}^{n}$, i.e.

$$
\left(e_{1} \cdots e_{n}\right) Y\left(e_{n} \cdots e_{1}\right)=-Y .
$$

There exist algebra isomorphisms

$$
\mathbb{C} l_{n} \cong \begin{cases}\operatorname{End}\left(\mathbb{C}^{2^{k}}\right) & \text { if } n=2 k, \\ \operatorname{End}\left(\mathbb{C}^{2^{k}}\right) \oplus \operatorname{End}\left(\mathbb{C}^{2^{k}}\right) & \text { if } n=2 k+1,\end{cases}
$$

and the space of (complex) spinors is defined to be

$$
\Delta_{n}:=\mathbb{C}^{2^{k}}=\underbrace{\mathbb{C}^{2} \otimes \ldots \otimes \mathbb{C}^{2}}_{k \text { times }} .
$$

The map

$$
\kappa: \mathbb{C} l_{n} \longrightarrow \operatorname{End}\left(\mathbb{C}^{2^{k}}\right)
$$


is defined to be either the aforementioned isomorphism for $n$ even, or the isomorphism followed by the projection onto the first summand for $n$ odd. In order to make $\kappa$ explicit, consider the following matrices

$$
I d=\left(\begin{array}{ll}
1 & 0 \\
0 & 1
\end{array}\right), \quad g_{1}=\left(\begin{array}{ll}
i & 0 \\
0 & -i
\end{array}\right), \quad g_{2}=\left(\begin{array}{ll}
0 & i \\
i & 0
\end{array}\right), \quad T=\left(\begin{array}{ll}
0 & -i \\
i & 0
\end{array}\right) .
$$

In terms of the generators $e_{1}, \ldots, e_{n}$ of the Clifford algebra, $\kappa$ can be described explicitly as follows,

$$
\begin{aligned}
e_{1} & \mapsto I d \otimes I d \otimes \ldots \otimes I d \otimes I d \otimes g_{1}, \\
e_{2} & \mapsto I d \otimes I d \otimes \ldots \otimes I d \otimes I d \otimes g_{2}, \\
e_{3} & \mapsto I d \otimes I d \otimes \ldots \otimes I d \otimes g_{1} \otimes T, \\
e_{4} & \mapsto I d \otimes I d \otimes \ldots \otimes I d \otimes g_{2} \otimes T, \\
\vdots & \\
e_{2 k-1} & \mapsto g_{1} \otimes T \otimes \ldots \otimes T \otimes T \otimes T, \\
e_{2 k} & \mapsto g_{2} \otimes T \otimes \ldots \otimes T \otimes T \otimes T,
\end{aligned}
$$

and, if $n=2 k+1$,

$$
e_{2 k+1} \mapsto i T \otimes T \otimes \ldots \otimes T \otimes T \otimes T \text {. }
$$

The vectors

$$
u_{+1}=\frac{1}{\sqrt{2}}(1,-i) \quad \text { and } \quad u_{-1}=\frac{1}{\sqrt{2}}(1, i),
$$

form a unitary basis of $\mathbb{C}^{2}$ with respect to the standard Hermitian product. Thus,

$$
\mathcal{B}=\left\{u_{\varepsilon_{1}, \ldots, \varepsilon_{k}}=u_{\varepsilon_{1}} \otimes \ldots \otimes u_{\varepsilon_{k}} \mid \varepsilon_{j}= \pm 1, j=1, \ldots, k\right\},
$$

is a unitary basis of $\Delta_{n}=\mathbb{C}^{2^{k}}$ with respect to the naturally induced Hermitian product. We will denote inner and Hermitian products (as well as Riemannian and Hermitian metrics) by the same symbol $\langle\cdot, \cdot\rangle$ trusting that the context will make clear which product is being used.

A quaternionic structure $\alpha$ on $\mathbb{C}^{2}$ is given by

$$
\alpha\left(\begin{array}{l}
z_{1} \\
z_{2}
\end{array}\right)=\left(\begin{array}{c}
-\bar{z}_{2} \\
\bar{z}_{1}
\end{array}\right)
$$

and a real structure $\beta$ on $\mathbb{C}^{2}$ is given by

$$
\beta\left(\begin{array}{l}
z_{1} \\
z_{2}
\end{array}\right)=\left(\begin{array}{l}
\bar{z}_{1} \\
\bar{z}_{2}
\end{array}\right)
$$

Following [6. p. 31], the real and quaternionic structures $\gamma_{n}$ on $\Delta_{n}=\left(\mathbb{C}^{2}\right)^{\otimes[n / 2]}$ are built as follows

$$
\begin{array}{lll}
\gamma_{n}=(\alpha \otimes \beta)^{\otimes 2 k} & \text { if } n=8 k, 8 k+1 & \text { (real), } \\
\gamma_{n}=\alpha \otimes(\beta \otimes \alpha)^{\otimes 2 k} & \text { if } n=8 k+2,8 k+3 & \text { (quaternionic), } \\
\gamma_{n}=(\alpha \otimes \beta)^{\otimes 2 k+1} & \text { if } n=8 k+4,8 k+5 & \text { (quaternionic), } \\
\gamma_{n}=\alpha \otimes(\beta \otimes \alpha)^{\otimes 2 k+1} & \text { if } n=8 k+6,8 k+7 & \text { (real). }
\end{array}
$$

The Spin group $\operatorname{Spin}(n) \subset C l_{n}$ is the subset

$$
\operatorname{Spin}(n)=\left\{x_{1} x_{2} \cdots x_{2 l-1} x_{2 l}\left|x_{j} \in \mathbb{R}^{n},\right| x_{j} \mid=1, l \in \mathbb{N}\right\},
$$

endowed with the product of the Clifford algebra. It is a Lie group and its Lie algebra is

$$
\mathfrak{s p i n}(n)=\operatorname{span}\left\{e_{i} e_{j} \mid 1 \leq i<j \leq n\right\} .
$$


The restriction of $\kappa$ to $\operatorname{Spin}(n)$ defines the Lie group representation

$$
\kappa_{n}:=\left.\kappa\right|_{\operatorname{Spin}(n)}: \operatorname{Spin}(n) \longrightarrow G L\left(\Delta_{n}\right),
$$

which is, in fact, special unitary. We have the corresponding Lie algebra representation

$$
\kappa_{n_{*}}: \mathfrak{s p i n}(n) \longrightarrow \mathfrak{g l}\left(\Delta_{n}\right) .
$$

Recall that the Spin group $\operatorname{Spin}(n)$ is the universal double cover of $S O(n), n \geq 3$. For $n=2$ we consider $\operatorname{Spin}(2)$ to be the connected double cover of $S O(2)$. The covering map will be denoted by

$$
\lambda_{n}: \operatorname{Spin}(n) \rightarrow S O(n) \subset G L\left(\mathbb{R}^{n}\right) .
$$

Its differential is given by $\lambda_{n_{*}}\left(e_{i} e_{j}\right)=2 E_{i j}$, where $E_{i j}=e_{i}^{*} \otimes e_{j}-e_{j}^{*} \otimes e_{i}$ is the standard basis of the skew-symmetric matrices, and $e^{*}$ denotes the metric dual of the vector $e$. Furthermore, we will abuse the notation and also denote by $\lambda_{n}$ the induced representation on the exterior algebra $\wedge^{*} \mathbb{R}^{n}$.

By means of $\kappa$, we have the Clifford multiplication

$$
\begin{aligned}
\mu_{n}: \mathbb{R}^{n} \otimes \Delta_{n} & \longrightarrow \Delta_{n} \\
x \otimes \phi & \mapsto \mu_{n}(x \otimes \phi)=x \cdot \phi:=\kappa(x)(\phi) .
\end{aligned}
$$

The Clifford multiplication $\mu_{n}$ is skew-symmetric with respect to the Hermitian product

$$
\left\langle x \cdot \phi_{1}, \phi_{2}\right\rangle=\left\langle\mu_{n}\left(x \otimes \phi_{1}\right), \phi_{2}\right\rangle=-\left\langle\phi_{1}, \mu_{n}\left(x \otimes \phi_{2}\right)\right\rangle=-\left\langle\phi_{1}, x \cdot \phi_{2}\right\rangle,
$$

is $\operatorname{Spin}(n)$-equivariant and can be extended to a $\operatorname{Spin}(n)$-equivariant map

$$
\begin{aligned}
\mu_{n}: \bigwedge^{*}\left(\mathbb{R}^{n}\right) \otimes \Delta_{n} & \longrightarrow \Delta_{n} \\
\omega \otimes \psi & \mapsto \omega \cdot \psi
\end{aligned}
$$

When $n$ is even, we define the following involution

$$
\begin{aligned}
\Delta_{n} & \longrightarrow \Delta_{n} \\
\psi & \mapsto(-i)^{\frac{n}{2}} \operatorname{Vol}_{n} \cdot \psi .
\end{aligned}
$$

The \pm 1 eigenspace of this involution is denoted $\Delta_{n}^{ \pm}$. These spaces have equal dimension and are irreducible representations of $\operatorname{Spin}(n)$. Note that our definition differs from the one given in $\left[\underline{6}\right.$ by a $(-1)^{\frac{n}{2}}$. The reason for this difference is that we want the spinor $u_{1, \ldots, 1}$ to be always positive. In this case, we will denote the two representations by

$$
\kappa_{n}^{ \pm}: \operatorname{Spin}(n) \longrightarrow G L\left(\Delta_{n}^{ \pm}\right)
$$

Note that while these representations are irreducible, they are not faithful, with kernels isomorphic to $\mathbb{Z}_{2}$ if $n \neq 4$.

Now, we summarize some results about real representations of $C l_{r}^{0}$ in the next table (cf. 8 $)$ ). Here $d_{r}$ denotes the dimension of an irreducible representation of $C l_{r}^{0}$ and $v_{r}$ the number of distinct non-trivial irreducible representations. Let $\tilde{\Delta}_{r}$ denote the irreducible representation of $C l_{r}^{0}$ for $r \not \equiv 0(\bmod 4)$ and $\tilde{\Delta}_{r}^{ \pm}$ 
denote the irreducible representations for $r \equiv 0(\bmod 4)$.

\begin{tabular}{|c|c|c|c|c|}
\hline$r(\bmod 8)$ & $d_{r}$ & $C l_{r}^{0}$ & $\tilde{\Delta}_{r} / \tilde{\Delta}_{r}^{ \pm} \cong \mathbb{R}^{d_{r}}$ & $v_{r}$ \\
\hline 1 & $2^{\left\lfloor\frac{r}{2}\right\rfloor}$ & $\mathbb{R}\left(d_{r}\right)$ & $\mathbb{R}^{d_{r}}$ & 1 \\
\hline 2 & $2^{\frac{r}{2}}$ & $\mathbb{C}\left(d_{r} / 2\right)$ & $\mathbb{C}^{d_{r} / 2}$ & 1 \\
\hline 3 & $2^{\left\lfloor\frac{r}{2}\right\rfloor+1}$ & $\mathbb{H}\left(d_{r} / 4\right)$ & $\mathbb{H}^{d_{r} / 4}$ & 1 \\
\hline 4 & $2^{\frac{r}{2}}$ & $\mathbb{H}\left(d_{r} / 4\right) \oplus \mathbb{H}\left(d_{r} / 4\right)$ & $\mathbb{H}^{d_{r} / 4}$ & 2 \\
\hline 5 & $2^{\left\lfloor\frac{r}{2}\right\rfloor+1}$ & $\mathbb{H}\left(d_{r} / 4\right)$ & $\mathbb{H}^{d_{r} / 4}$ & 1 \\
\hline 6 & $2^{\frac{r}{2}}$ & $\mathbb{C}\left(d_{r} / 2\right)$ & $\mathbb{C}^{d_{r} / 2}$ & 1 \\
\hline 7 & $2^{\left\lfloor\frac{r}{2}\right\rfloor}$ & $\mathbb{R}\left(d_{r}\right)$ & $\mathbb{R}^{d_{r}}$ & 1 \\
\hline 8 & $2^{\frac{r}{2}-1}$ & $\mathbb{R}\left(d_{r}\right) \oplus \mathbb{R}\left(d_{r}\right)$ & $\mathbb{R}^{d_{r}}$ & 2 \\
\hline
\end{tabular}

Table 1

\subsection{Maximal Torus of $\operatorname{Spin}(r)$}

In this subsection, we recall explicit expressions for elements of the maximal torus of the Spin group since it will be useful to consider paths within such torus.

The rotation

$$
\left(\begin{array}{ccccc}
\cos \left(\theta_{1}\right) & -\sin \left(\theta_{1}\right) & & & \\
\sin \left(\theta_{1}\right) & \cos \left(\theta_{1}\right) & & & \\
& & 1 & & \\
& & & \ddots & \\
& & & & 1
\end{array}\right)_{r \times r}
$$

can be achieved by using the element

$$
e_{1}\left(-\cos \left(\theta_{1} / 2\right) e_{1}+\sin \left(\theta_{1} / 2\right) e_{2}\right)=\cos \left(\theta_{1} / 2\right)+\sin \left(\theta_{1} / 2\right) e_{1} e_{2} \in \operatorname{Spin}(r)
$$

as follows

$$
\begin{gathered}
\left(\cos \left(\theta_{1} / 2\right)+\sin \left(\theta_{1} / 2\right) e_{1} e_{2}\right) y\left(\cos \left(\theta_{1} / 2\right)-\sin \left(\theta_{1} / 2\right) e_{1} e_{2}\right) \\
=\left(y_{1} \cos \left(\theta_{1}\right)-y_{2} \sin \left(\theta_{1}\right)\right) e_{1}+\left(y_{1} \sin \left(\theta_{1}\right)+y_{2} \cos \left(\theta_{1}\right) e_{2}+y_{3} e_{3}+\cdots+y_{r} e_{r},\right.
\end{gathered}
$$

for $y=y_{1} e_{1}+\cdots+y_{r} e_{r} \in \mathbb{R}^{r}$. Thus, we see that the corresponding elements in $\operatorname{Spin}(r)$ are exactly

$$
\pm\left(\cos \left(\theta_{1} / 2\right)+\sin \left(\theta_{1} / 2\right) e_{1} e_{2}\right) .
$$

Furthermore, we can see that a maximal torus of $\operatorname{Spin}(r)$ consists of elements of the form

$$
t\left(\theta_{1}, \ldots, \theta_{\left[\frac{r}{2}\right]}\right)=\prod_{j=1}^{\left[\frac{r}{2}\right]}\left(\cos \left(\theta_{j} / 2\right)+\sin \left(\theta_{j} / 2\right) e_{2 j-1} e_{2 j}\right),
$$

noting that the parameters $\theta_{j}$ must now run between 0 and $4 \pi$. Furthermore, using the explicit description of the isomorphisms given above, we see that

$$
\begin{aligned}
\left(\cos \left(\theta_{1} / 2\right)+\sin \left(\theta_{1} / 2\right) e_{1} e_{2}\right) \cdot u_{\varepsilon_{1}, \ldots \varepsilon_{k}} & =\cos \left(\theta_{1} / 2\right) u_{\varepsilon_{1}, \ldots \varepsilon_{k}}+\sin \left(\theta_{1} / 2\right) e_{1} e_{2} \cdot u_{\varepsilon_{1}, \ldots \varepsilon_{k}} \\
& =\cos \left(\theta_{1} / 2\right) u_{\varepsilon_{1}, \ldots \varepsilon_{k}}+i \varepsilon_{k} \sin \left(\theta_{1} / 2\right) u_{\varepsilon_{1}, \ldots \varepsilon_{k}} \\
& =\left(\cos \left(\theta_{1} / 2\right)+i \varepsilon_{k} \sin \left(\theta_{1} / 2\right)\right) u_{\varepsilon_{1}, \ldots \varepsilon_{k}} \\
& =e^{i \frac{\varepsilon_{k} \theta_{1}}{2}} u_{\varepsilon_{1}, \ldots \varepsilon_{k}}
\end{aligned}
$$


and similarly,

$$
t\left(\theta_{1}, \ldots, \theta_{\left[\frac{r}{2}\right]}\right) \cdot u_{\varepsilon_{1}, \ldots \varepsilon_{\left[\frac{r}{2}\right]}}=e^{\frac{i}{2} \sum_{j=1}^{\left[\frac{r}{2}\right]} \varepsilon_{k+1-j} \theta_{j}} \cdot u_{\varepsilon_{1}, \ldots \varepsilon_{k}} .
$$

Thus, the basis vectors $u_{\varepsilon_{1}, \ldots \varepsilon_{k}}$ are weight vectors of the standard spin representation with weight

$$
\frac{1}{2} \sum_{j=1}^{\left[\frac{r}{2}\right]} \varepsilon_{\left[\frac{r}{2}\right]+1-j} \theta_{j},
$$

which in coordinate vectors are the well known expressions

$$
\left( \pm \frac{1}{2}, \pm \frac{1}{2}, \cdots, \pm \frac{1}{2}\right)
$$

Moreover, in terms of the (appropriately ordered) basis $\mathcal{B}$, the matrix associated to an element $t\left(\theta_{1}, \ldots, \theta_{\left[\frac{r}{2}\right]}\right)$ is

$$
\left(\begin{array}{ccccc}
e^{\frac{i}{2}\left(\theta_{1}+\theta_{2}+\cdots+\theta_{\left[\frac{r}{2}\right]}\right)} & & & & \\
& e^{\frac{i}{2}\left(-\theta_{1}+\theta_{2}+\cdots+\theta_{\left[\frac{r}{2}\right]}\right)} & & & \\
& e^{\frac{i}{2}\left(\theta_{1}-\theta_{2}+\cdots+\theta_{\left[\frac{r}{2}\right]}\right)} & & & \\
& & \ddots & & \\
& & e^{\frac{i}{2}\left(-\theta_{1}-\theta_{2}+\cdots+\theta_{\left[\frac{r}{2}\right]}\right)} & \\
& & & \ddots & \\
e^{\frac{i}{2}\left(-\theta_{1}-\theta_{2}-\cdots-\theta_{\left[\frac{r}{2}\right]}\right)}
\end{array}\right) .
$$

Note that, when $r$ is even, $\Delta_{r}^{+}$is generated by the basis vectors $u_{\varepsilon_{1}, \ldots \varepsilon_{\frac{r}{2}}}$ with an even number of $\varepsilon_{j}$ equal to -1 , and $\Delta_{r}^{-}$is generated by the basis vectors $u_{\varepsilon_{1}, \ldots \varepsilon_{r}}$ with an odd number of $\varepsilon_{j}$ equal to -1 . Therefore, after reordering the basis, the matrix above can be split into two blocks of equal size: one block in which the exponents contain an even number of negative signs

$$
\left(\begin{array}{cccc}
e^{\frac{i}{2}\left(\theta_{1}+\theta_{2}+\cdots+\theta_{\frac{r}{2}}\right)} & & & \\
& e^{\frac{i}{2}\left(-\theta_{1}-\theta_{2}+\cdots+\theta_{\frac{r}{2}}\right)} & & \\
& & e^{\frac{i}{2}\left(-\theta_{1}+\theta_{2}-\cdots+\theta_{\frac{r}{2}}\right)} & \\
& & \ddots
\end{array}\right)
$$

and another block in which the exponents contain an odd number of negative signs

$$
\left(\begin{array}{cccc}
e^{\frac{i}{2}\left(-\theta_{1}+\theta_{2}+\cdots+\theta_{\frac{r}{2}}\right)} & & & \\
& e^{\frac{i}{2}\left(\theta_{1}-\theta_{2}+\cdots+\theta_{\frac{r}{2}}\right)} & & \\
& & e^{\frac{i}{2}\left(\theta_{1}+\theta_{2}-\theta_{3}+\cdots+\theta_{\frac{r}{2}}\right)} & \\
& & \ddots
\end{array}\right)
$$

\subsection{Even Clifford structures}

\subsubsection{Linear almost even-Clifford Hermitian structures}

Definition 2.1 Let $N \in \mathbb{N}$ and $\left(e_{1}, \ldots, e_{r}\right)$ an orthonormal frame of $\mathbb{R}^{r}$.

- A linear even-Clifford structure of rank $r$ on $\mathbb{R}^{N}$ is an algebra representation

$$
\Phi: C l_{r}^{0} \longrightarrow \operatorname{End}\left(\mathbb{R}^{N}\right) .
$$


- A linear even-Clifford Hermitian structure of rank $r$ on $\mathbb{R}^{N}$ (endowed with a positive definite inner product) is a linear even-Clifford structure of rank $r$ such that each bivector $e_{i} e_{j}, 1 \leq i<j \leq r$, is mapped to an skew-symmetric endomorphism $\Phi\left(e_{i} e_{j}\right)=J_{i j}$.

\section{Remarks.}

- Note that

$$
J_{i j}^{2}=-\operatorname{Id}_{\mathbb{R}^{N}}
$$

- Given a linear even-Clifford structure of $\operatorname{rank} r$ on $\mathbb{R}^{N}$, we can average the standard inner product $\langle$, on $\mathbb{R}^{N}$ as follows

$$
(X, Y)=\sum_{k=1}^{[r / 2]}\left[\sum_{1 \leq i_{1}<\ldots<i_{2 k}<r}\left\langle\Phi\left(e_{i_{1} \ldots i_{2 k}}\right)(X), \Phi\left(e_{i_{1} \ldots i_{2 k}}\right)(Y)\right\rangle\right],
$$

where $\left(e_{1}, \ldots, e_{r}\right)$ is an orthonormal frame of $\mathbb{R}^{r}$, so that the linear even-Clifford structure is Hermitian with respect to the averaged inner product.

- Given a linear even-Clifford Hermitian structure structure of rank $r$, the subalgebra $\mathfrak{s p i n}(r)$ is mapped injectively into the skew-symmetric endomorphisms $\operatorname{End}^{-}\left(\mathbb{R}^{N}\right)$.

\subsubsection{Branching of $\mathbb{R}^{N}$}

From now on, we will denote by $\operatorname{Id}_{n}$ the identity endomorphism of a real/complex $n$-dimensional vector space.

First, let us assume $r \not \equiv 0(\bmod 4), r>1$. In this case, $\mathbb{R}^{N}$ decomposes into a sum of irreducible representations of $C l_{r}^{0}$. Since $C l_{r}^{0}$ is simple, its irreducible representations are either trivial or the standard representation $\tilde{\Delta}_{r}$ of $C l_{r}^{0}$ (cf. $[\underline{8})$. Due to $\left(\mathbb{1}\right.$, there are no trivial summands $\mathbb{R}^{N}$, i.e.

$$
\mathbb{R}^{N}=\mathbb{R}^{m} \otimes \tilde{\Delta}_{r}
$$

for some $m \in \mathbb{N}$. Thus, we see that $\mathfrak{s p i n}(r)$ has an isomorphic image

$$
\widehat{\mathfrak{s p i n}(r)}:=\operatorname{Id}_{m} \otimes \kappa_{r^{*}}(\mathfrak{s p i n}(r)) \subset \mathfrak{s o}\left(d_{r} m\right) .
$$

Secondly, let us assume $r \equiv 0(\bmod 4)$. Recall that if $\hat{\Delta}_{r}$ is the irreducible representation of $C l_{r}$, then by restricting this representation to $C l_{r}^{0}$ it splits as the sum of two inequivalent irreducible representations

$$
\hat{\Delta}_{r}=\tilde{\Delta}_{r}^{+} \oplus \tilde{\Delta}_{r}^{-} .
$$

Since $\mathbb{R}^{N}$ is a representation of $C l_{r}^{0}$ satisfying (1), there are no trivial summands in $\mathbb{R}^{N}$ and

$$
\mathbb{R}^{N}=\mathbb{R}^{m_{1}} \otimes \tilde{\Delta}_{r}^{+} \oplus \mathbb{R}^{m_{2}} \otimes \tilde{\Delta}_{r}^{-}
$$

for some $m_{1}, m_{2} \in \mathbb{N}$. By restricting this representation to $\mathfrak{s p i n}(r) \subset C l_{r}^{0}$, consider the isomorphic image

$$
\widehat{\mathfrak{s p i n}(r)}:=\left\{\operatorname{Id}_{m_{1}} \otimes \xi^{+} \oplus \operatorname{Id}_{m_{2}} \otimes \xi^{-} \mid \xi \in \mathfrak{s p i n}(r), \xi^{+}=\kappa_{r^{*}}^{+}(\xi), \xi^{-}=\kappa_{r^{*}}^{-}(\xi)\right\} .
$$

\subsubsection{Almost even-Clifford Hermitian manifolds}

Definition 2.2 Let $r \geq 2$.

- A rank $r$ almost even-Clifford structure on a smooth manifold $M$ is a smoothly varying choice of a rank $r$ linear even-Clifford structure on each tangent space of $M$. 
- A smooth manifold carrying an almost even-Clifford structure will be called an almost even-Clifford manifold.

- A rank $r$ almost even-Clifford Hermitian structure on a Riemannian manifold $M$ is a smoothly varying choice of a linear even-Clifford Hermitian structure on each tangent space of $M$.

- A Riemannian manifold carrying an almost even-Clifford Hermitian structure will be called an almost even-Clifford Hermitian manifold.

Remark. Our definition of almost even-Clifford hermintian structure implies that in [10].

\section{Complexifications, structure groups and fundamental groups}

In this section we will study the complexification $\mathbb{R}^{N} \otimes \mathbb{C}$ and its decomposition as a representation of the normalizers $\mathcal{N}_{\mathfrak{s o}(N)}^{0}(S)$ (cf. 3.1), where $S$ is one of the homomorphic images of $\mathfrak{s p i n}(r)$ described in Subsection 2.3.2. Once we have described such complexifications and decompositions, we will compute the (connected components of the identity of the) structure groups determined by linear even-Clifford structures. They must be closed Lie subgroups of $S O(N)$ whose Lie algebra is the aforementioned normalizer. They are actually isomorphic to finite quotients of products of classical compact Lie groups (or real lines) with Spin $(r)$ (cf. 3.2). Along the way, we will also compute their fundamental groups to be used in the last section. All of this will be done in case by case analysis.

Along the way, we will introduce notation that will enable us to state Theorems 3.1, 3.2 and 3.3. The main steps in each case are the following:

1. Complexification.

- Identify $\mathbb{R}^{N} \otimes \mathbb{C}$ as a representation

$$
G \times \operatorname{Spin}(r) \stackrel{\rho}{\rightarrow} S O(N) \subset \operatorname{Aut}\left(\mathbb{R}^{N} \times \mathbb{C}\right),
$$

where $G$ denotes a (semi-)simple compact Lie group or $S O(2)$.

2. Structure group.

- Compute the image of $\rho, \operatorname{Im}(\rho)$.

- Compute $\operatorname{ker}(\rho)$ to get

$$
\operatorname{Im}(\rho) \cong \frac{G \times \operatorname{Spin}(r)}{\operatorname{ker}(\rho)}
$$

3. Fundamental group.

- Identify the universal covering $\widetilde{\operatorname{Im}(\rho)} \stackrel{\tilde{\rho}}{\rightarrow} \operatorname{Im}(\rho)$.

- Compute the preimage of $\tilde{\rho}^{-1}\left(\operatorname{Id}_{N}\right)$ to obtain an explicit description of the fundamental group $\pi_{1}(\operatorname{Im}(\rho))$.

First, let us recall the following table [2], whose entries' precise description will be recalled in each case.

\begin{tabular}{|c|c|c|c|}
\hline$r(\bmod 8)$ & $N$ & $\mathcal{C}_{\mathfrak{s o}(N)}(\widehat{\mathfrak{s p i n}(r)})$ & $\left.\mathcal{N}_{\mathfrak{s o}(N)}^{0} \widehat{(\mathfrak{s p i n}(r)}\right)$ \\
\hline 0 & $d_{r}\left(m_{1}+m_{2}\right)$ & $\widehat{\mathfrak{s o}\left(m_{1}\right)} \oplus \widehat{\mathfrak{s o}\left(m_{2}\right)}$ & $\left.\widehat{\mathfrak{s o}\left(m_{1}\right.}\right) \oplus \widehat{\mathfrak{s o}\left(m_{2}\right) \oplus \widehat{\mathfrak{s p i n}(r)}}$ \\
\hline 1,7 & $d_{r} m$ & $\widehat{\mathfrak{s o}(m)}$ & $\widehat{\mathfrak{s o}(m)} \oplus \widehat{\mathfrak{s p i n}(r)}$ \\
\hline 2,6 & $d_{r} m$ & $\widehat{\mathfrak{u}(m)}$ & $\widehat{\mathfrak{u}(m)} \oplus \widehat{\mathfrak{s p i n}(r)}$ \\
\hline 3,5 & $d_{r} m$ & $\widehat{\mathfrak{s p}(m)}$ & $\widehat{\mathfrak{s p}(m)} \oplus \widehat{\mathfrak{s p i n}(r)}$ \\
\hline 4 & $d_{r}\left(m_{1}+m_{2}\right)$ & $\widehat{\mathfrak{s p}\left(m_{1}\right)} \oplus \widehat{\mathfrak{s p}\left(m_{2}\right)}$ & $\widehat{\mathfrak{s p}\left(m_{1}\right)} \oplus \widehat{\mathfrak{s p}\left(m_{2}\right) \oplus \widehat{\mathfrak{s p i n}(r)}}$ \\
\hline
\end{tabular}

Table 2 


\section{1 $r \equiv 1,7(\bmod 8)$}

Complexification. In this case, $\tilde{\Delta}_{r}$ is the subspace of $\Delta_{r}$ fixed by the corresponding real structure $\gamma_{r}$, i.e.

$$
\tilde{\Delta}_{r} \otimes \mathbb{C}=\Delta_{r} .
$$

The centralizer subalgebra of $\widehat{\mathfrak{s p i n}(r)}$ in $\mathfrak{s o}(N)$ is

$$
C_{\mathfrak{s o}(N)}(\widehat{\mathfrak{s p i n}(r)})=\mathfrak{s o}(m) \otimes \operatorname{Id}_{\tilde{\Delta}_{r}}=: \widehat{\mathfrak{s o}(m)},
$$

where $N=d_{r} m$. If $z \in \mathbb{C}, v \otimes \psi \in \mathbb{R}^{m} \otimes \tilde{\Delta}_{r}$ and $A \in \mathfrak{s o}(m)$,

$$
\left(A \otimes \operatorname{Id}_{\tilde{\Delta}_{r}}\right)(z v \otimes \psi)=z A v \otimes \psi,
$$

which means

$$
\left(\mathbb{R}^{m} \otimes \tilde{\Delta}_{r}\right) \otimes \mathbb{C}=\mathbb{C}^{m} \otimes \Delta_{r},
$$

where $\mathbb{C}^{m}$ denotes the standard complex representation of $S O(m)$. Thus, we have a representation

$$
S O(m) \times \operatorname{Spin}(r) \stackrel{\rho}{\rightarrow} S O(N) \subset \operatorname{Aut}\left(\mathbb{C}^{m} \otimes \Delta_{r}\right) .
$$

Structure group. Since $\widehat{\mathfrak{s o}(m)}$ and $\widehat{\mathfrak{s p i n}(r)}$ commute with each other, we can take separately the exponentials of their elements within $\mathbb{C}(N)$. The exponential of $A \otimes \operatorname{Id}_{\tilde{\Delta}_{r}} \in \widehat{\mathfrak{s o}(m)}$ gives

$$
e^{A} \otimes \operatorname{Id}_{\tilde{\Delta}_{r}} \in \widehat{S O(m)}:=S O(m) \otimes \operatorname{Id}_{\tilde{\Delta}_{r}} \cong S O(m) .
$$

On the other hand, if $\operatorname{Id}_{m} \otimes \xi \in \widehat{\mathfrak{s p i n}(r)}$, its exponential is

$$
\operatorname{Id}_{m} \otimes e^{\xi} \in \operatorname{Id}_{m} \otimes \kappa(\operatorname{Spin}(r))=: \widehat{\operatorname{Spin}(r)} \cong \operatorname{Spin}(r),
$$

since $\operatorname{Spin}(r)$ is represented faithfully on $\Delta_{r}$. The image of $S O(m) \times \operatorname{Spin}(r)$ in $S O(N)$ under the aforementioned representation is

$$
\mathcal{N}_{S O(N)}^{0}(\widehat{\operatorname{Spin}(r)})=\widehat{\operatorname{SO}(m)} \widehat{\operatorname{Spin}(r)},
$$

the subgroup of all possible products of elements of the two subgroups, i.e. we have

$$
S O(m) \times \operatorname{Spin}(r) \stackrel{\rho}{\rightarrow} \widehat{\operatorname{SO}(m)} \widehat{\operatorname{Spin}(r)} \subset S O(N) .
$$

Now we need to find $\operatorname{ker}(\rho)$ and identify $\widehat{\operatorname{SO(m})} \widehat{\operatorname{Spin}(r)}$ as a quotient

$$
\widehat{\operatorname{SO}(m)} \widehat{\operatorname{Spin}(r)} \cong \frac{S O(m) \times \operatorname{Spin}(r)}{\operatorname{ker}(\rho)} .
$$

If there are elements $g \in S O(m)$ and $h \in S p i n(r)$ such that

$$
\rho(g, h)=\operatorname{Id}_{N},
$$

then

$$
\widehat{\operatorname{Spin}(r)} \ni \rho\left(\operatorname{Id}_{m}, h\right)=\rho(g, 1)^{-1} \in \widehat{S O(m)} .
$$

Since $\rho\left(\operatorname{Id}_{m}, h\right)$ commutes with every element of $\left.\widehat{\operatorname{Spin}(r}\right)$, it belongs to its center $\left.Z(\widehat{\operatorname{Spin}(r})\right) \cong Z(\operatorname{Spin}(r))=$ $\mathbb{Z}_{2}=\{ \pm 1\}$. Note that $-1 \in \operatorname{Spin}(r)$ maps to $-\operatorname{Id}_{\Delta_{r}}$ under the $\operatorname{Spin}(r)$ representation $\Delta_{r}$, and $\left(\operatorname{Id}_{m},-1\right)$ maps to $-\operatorname{Id}_{m} \otimes \operatorname{Id}_{\Delta_{r}} \in S O(N)$ under $\rho$. Moreover, $-\operatorname{Id}_{m} \otimes \operatorname{Id}_{\Delta_{r}}$ belongs to $\widehat{S O(m)}$ only if $m$ is even. Thus, $\operatorname{ker}(\rho)=\left\{ \pm\left(\operatorname{Id}_{m}, 1\right)\right\}=\mathbb{Z}_{2}$ and

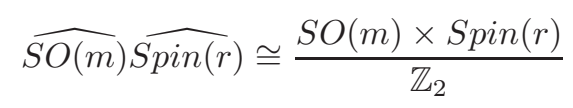


if $m$ is even, and $\operatorname{ker}(\rho)=\left\{\left(\operatorname{Id}_{m}, 1\right)\right\}$,

$$
\widehat{\operatorname{SO}(m)} \widehat{\operatorname{Spin}(r)} \cong S O(m) \times \operatorname{Spin}(r)
$$

if $m$ is odd.

Fundamental group. Clearly, we only need to deal with the case when $m$ is even.

- If $m \geq 4$, let $\tilde{\rho}$ denote the following composition

$$
\begin{aligned}
\operatorname{Spin}(m) & \times \operatorname{Spin}(r) \\
& \downarrow \\
S O(m) & \times \operatorname{Spin}(r) \\
& \downarrow \\
S O(m) & \times_{\mathbb{Z}_{2}} \operatorname{Spin}(r)
\end{aligned}
$$

We need to find all the elements of $\operatorname{Spin}(m) \times \operatorname{Spin}(r)$ that map to

$$
\pm\left(\operatorname{Id}_{m}, 1\right)
$$

The elements of $\operatorname{Spin}(m) \times \operatorname{Spin}(r)$ that map to $\left(\operatorname{Id}_{m}, 1\right) \in S O(m) \times \operatorname{Spin}(r)$ are

$$
( \pm 1,1)
$$

and the elements of $\operatorname{Spin}(m) \times \operatorname{Spin}(r)$ that map to $\left(-\mathrm{Id}_{m},-1\right) \in S O(m) \times \operatorname{Spin}(r)$ are

$$
\left( \pm \operatorname{vol}_{m},-1\right)
$$

i.e.

$$
\begin{aligned}
\operatorname{ker}(\tilde{\rho}) & =\left\{(1,1),(-1,1),\left(\operatorname{vol}_{m},-1\right),\left(-\operatorname{vol}_{m},-1\right)\right\} . \\
\pi_{1}(\widehat{\operatorname{SO}(m)} \widehat{\operatorname{Spin}(r)}) & \cong \begin{cases}\mathbb{Z}_{2} \oplus \mathbb{Z}_{2} & \text { if } m \equiv 0(\bmod 4) \\
\mathbb{Z}_{4} & \text { if } m \equiv 2(\bmod 4) .\end{cases}
\end{aligned}
$$

- If $m=2$, let $\tilde{\rho}$ denote the following composition

$$
\begin{gathered}
\mathbb{R} \times \operatorname{Spin}(r) \\
\downarrow \\
\qquad O(2) \times \operatorname{Spin}(r) \\
\downarrow \\
\quad \downarrow \\
S O(2) \times \mathbb{Z}_{2} \operatorname{Spin}(r)
\end{gathered}
$$

Similarly,

$$
\begin{aligned}
\widehat{\operatorname{ker}(\tilde{\rho})} & =\{(2 k \pi, 1) \mid k \in \mathbb{Z}\} \cup\{((2 k+1) \pi,-1) \mid k \in \mathbb{Z}\}, \\
\pi_{1}(\widehat{\operatorname{SO}(2)} \widehat{\operatorname{Spin}(r)}) & \cong \mathbb{Z}
\end{aligned}
$$

\section{$3.2 r \equiv 0(\bmod 8)$}

Complexification. In this case, $\tilde{\Delta}_{r}^{+}$and $\tilde{\Delta}_{r}^{-}$are the subspaces of $\Delta_{r}^{+}$and $\Delta_{r}^{-}$fixed by the corresponding real structure $\gamma_{r}$ mentioned in Section [2, and

$$
\begin{aligned}
& \tilde{\Delta}_{r}^{+} \otimes \mathbb{C}=\Delta_{r}^{+}, \\
& \tilde{\Delta}_{r}^{-} \otimes \mathbb{C}=\Delta_{r}^{-} .
\end{aligned}
$$


The centralizer subalgebra of $\widehat{\mathfrak{s p i n}(r)}$ is

$$
\begin{aligned}
& C_{\mathfrak{s o}(N)}(\widehat{\mathfrak{s p i n}(r)})=\widehat{\mathfrak{s o}\left(m_{1}\right)} \oplus \widehat{\mathfrak{s o}\left(m_{2}\right)} \\
& =\left(\begin{array}{ll}
\widehat{\mathfrak{s o}\left(m_{1}\right)} & \\
& \widehat{\mathfrak{s o}\left(m_{2}\right)}
\end{array}\right) \\
& :=\left(\begin{array}{ll}
\mathfrak{s o}\left(m_{1}\right) \otimes \operatorname{Id}_{\tilde{\Delta}_{r}^{+}} & \\
& \mathfrak{s o}\left(m_{2}\right) \otimes \operatorname{Id}_{\tilde{\Delta}_{r}^{-}}
\end{array}\right),
\end{aligned}
$$

where $N=d_{r}\left(m_{1}+m_{2}\right)$. If $A_{1} \in \mathfrak{s o}\left(m_{1}\right), A_{2} \in \mathfrak{s o}\left(m_{2}\right), z_{1}, z_{2} \in \mathbb{C}, v_{1} \otimes \psi_{1}+v_{2} \otimes \psi_{2} \in \mathbb{R}^{m_{1}} \otimes \tilde{\Delta}_{r}^{+} \oplus \mathbb{R}^{m_{1}} \otimes \tilde{\Delta}_{r}^{-}$,

$$
\left(\begin{array}{cc}
A_{1} \otimes \operatorname{Id}_{\tilde{\Delta}_{r}^{+}} & \\
& A_{2} \otimes \operatorname{Id}_{\tilde{\Delta}_{r}^{-}}
\end{array}\right)\left(\begin{array}{c}
z_{1} v_{1} \otimes \psi_{1} \\
z_{2} v_{2} \otimes \psi_{2}
\end{array}\right)=\left(\begin{array}{c}
z_{1} A_{1} v_{1} \otimes \psi_{1} \\
z_{2} A_{2} v_{2} \otimes \psi_{2}
\end{array}\right)
$$

which means

$$
\left(\mathbb{R}^{m_{1}} \otimes \tilde{\Delta}_{r}^{+} \oplus \mathbb{R}^{m_{2}} \otimes \tilde{\Delta}_{r}^{-}\right) \otimes \mathbb{C}=\mathbb{C}^{m_{1}} \otimes \Delta_{r}^{+} \oplus \mathbb{C}^{m_{2}} \otimes \Delta_{r}^{-},
$$

where $\mathbb{C}^{m_{1}}$ and $\mathbb{C}^{m_{2}}$ denote the standard complex representation of $\mathfrak{s o}\left(m_{1}\right)$ and $\mathfrak{s o}\left(m_{2}\right)$ respectively. Thus, we have a representation

$$
S O\left(m_{1}\right) \times S O\left(m_{2}\right) \times \operatorname{Spin}(r) \longrightarrow S O(N) \subset \operatorname{Aut}\left(\mathbb{C}^{m_{1}} \otimes \Delta_{r}^{+} \oplus \mathbb{C}^{m_{2}} \otimes \Delta_{r}^{-}\right) .
$$

Structure group. Since $\widehat{\mathfrak{s o}\left(m_{1}\right)} \oplus \widehat{\mathfrak{s o}\left(m_{2}\right)}$ and $\widehat{\mathfrak{s p i n}(r)}$ commute, we can take the exponentials of their elements separately within $\mathbb{C}(N)$. The exponential of

$$
\left(\begin{array}{cc}
A_{1} \otimes \operatorname{Id}_{\Delta_{r}^{+}} & \\
& A_{2} \otimes \operatorname{Id}_{\Delta_{r}^{-}}
\end{array}\right) \in \widehat{\mathfrak{s o}\left(m_{1}\right)} \oplus \widehat{\mathfrak{s o}\left(m_{2}\right)}
$$

is

$$
\begin{aligned}
\left(\begin{array}{cc}
e^{A_{1}} \otimes \operatorname{Id}_{\Delta_{r}^{+}} & \\
& e^{A_{2}} \otimes \operatorname{Id}_{\Delta_{r}^{-}}
\end{array}\right) & \in\left(\begin{array}{cc}
\left.\widehat{S O\left(m_{1}\right.}\right) & \left.\widehat{S O\left(m_{2}\right.}\right)
\end{array}\right) \\
& :=\left(\begin{array}{ll}
S O\left(m_{1}\right) \otimes \operatorname{Id}_{\Delta_{r}^{+}} & S O\left(m_{2}\right) \otimes \operatorname{Id}_{\Delta_{r}^{-}}
\end{array}\right) . \\
& =S \widehat{S\left(m_{1}\right)} \times \widehat{S O\left(m_{2}\right)} .
\end{aligned}
$$

On the other hand, if $\operatorname{Id}_{m_{1}} \otimes \xi^{+} \oplus \operatorname{Id}_{m_{2}} \otimes \xi^{-} \in \widehat{\mathfrak{s p i n}(r)}$, its exponential is

$$
\begin{aligned}
& \left(\begin{array}{cc}
\operatorname{Id}_{m_{1}} \otimes e^{\xi^{+}} & \\
& \operatorname{Id}_{m_{2}} \otimes e^{\xi^{-}}
\end{array}\right) \in\left(\begin{array}{ll}
\operatorname{Id}_{m_{1}} \otimes \kappa^{+}(\operatorname{Spin}(r)) & \\
& \operatorname{Id}_{m_{2}} \otimes \kappa^{-}(\operatorname{Spin}(r))
\end{array}\right)
\end{aligned}
$$

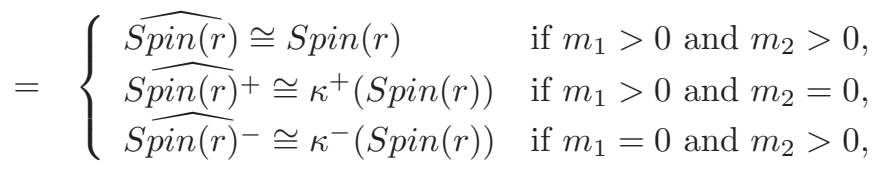

where the first representation of $\operatorname{Spin}(r)$ is faithful and the last two are not, with

$$
\widehat{\operatorname{Sin}(r)^{ \pm}} \cong \kappa^{ \pm}(\operatorname{Spin}(r)) \cong \frac{\operatorname{Spin}(r)}{\left\{1, \pm \operatorname{vol}_{r}\right\}}
$$

The image of $S O\left(m_{1}\right) \times S O\left(m_{2}\right) \times \operatorname{Spin}(r)$ in $S O(N)$ under the aforementioned representations are

$$
\begin{aligned}
& \left.\mathcal{N}_{S O(N)}^{0}(\widehat{\operatorname{Spin}(r)})=\left(\widehat{S O\left(m_{1}\right.}\right) \times \widehat{S O\left(m_{2}\right)}\right) \widehat{\operatorname{Spin}(r)}, \\
& \mathcal{N}_{S O(N)}^{0}(\widehat{\operatorname{Spin}(r)}+)=\widehat{S O\left(m_{1}\right)} \widehat{\operatorname{Spin}(r)}+, \\
& \mathcal{N}_{S O(N)}^{0}\left(\widehat{\operatorname{Sin}(r)^{-}}\right)=\widehat{S O\left(m_{2}\right)} \widehat{S} \widehat{\operatorname{pin}(r)^{-}} \text {, }
\end{aligned}
$$


respectively, i.e. in each case we have a map

$$
\begin{aligned}
& \left.S O\left(m_{1}\right) \times S O\left(m_{2}\right) \times S \sin (r) \stackrel{\rho}{\rightarrow}\left(\widehat{S O\left(m_{1}\right.}\right) \times \widehat{S O\left(m_{2}\right)}\right) \widehat{\operatorname{Spin}(r)} \subset S O(N), \\
& S O\left(m_{1}\right) \times \operatorname{Spin}(r) \stackrel{\rho}{\rightarrow} \widehat{S O\left(m_{1}\right)} \widehat{S p i n(r)}-\subset S O(N), \\
& S O\left(m_{2}\right) \times \operatorname{Spin}(r) \stackrel{\rho}{\rightarrow} S \widehat{S O\left(m_{2}\right)} \widehat{\operatorname{Spin}(r)}+\subset S O(N) .
\end{aligned}
$$

Now we need to find $\operatorname{ker}(\rho)$ in each case to identify the relevant group as a quotient.

- Case $m_{1}, m_{2}>0$. If there are elements $g_{i} \in S O\left(m_{i}\right)$ and $h \in \operatorname{Spin}(r)$ such that

$$
\rho\left(g_{1}, g_{2}, h\right)=\operatorname{Id}_{N}
$$

then

$$
\left.\left.\widehat{\operatorname{Spin}(r)} \ni \rho\left(\operatorname{Id}_{m_{1}}, \operatorname{Id}_{m_{2}}, h\right)=\rho\left(g_{1}, g_{2}, 1\right)^{-1} \in \widehat{S O\left(m_{1}\right.}\right) \times \widehat{S O\left(m_{2}\right.}\right) .
$$

Since $\rho\left(\operatorname{Id}_{m_{1}}, \operatorname{Id}_{m_{2}}, h\right)$ commutes with every element of $\widehat{\operatorname{Spin}(r)}$, it belongs to its center $Z(\widehat{\operatorname{Spin}(r)}) \cong$ $Z(\operatorname{Spin}(r))=\left\{1,-1, \operatorname{vol}_{r},-\operatorname{vol}_{r}\right\} \cong \mathbb{Z}_{2} \oplus \mathbb{Z}_{2}$. Note that

- the element -1 is mapped to $-\operatorname{Id}_{\Delta_{r}^{ \pm}}$in the $\operatorname{Spin}(r)$ representations $\Delta_{r}^{ \pm}$, and $\left(\operatorname{Id}_{m_{1}}, \operatorname{Id}_{m_{2}},-1\right)$ maps to $-\left(\operatorname{Id}_{m_{1}} \otimes \operatorname{Id}_{\Delta_{r}^{+}} \oplus \operatorname{Id}_{m_{2}} \otimes \operatorname{Id}_{\Delta_{r}^{-}}\right)^{+} \in S O(N)$; the element $-\left(\operatorname{Id}_{m_{1}} \otimes \operatorname{Id}_{\Delta_{r}^{+}} \oplus \operatorname{Id}_{m_{2}} \otimes \operatorname{Id}_{\Delta_{r}^{-}}\right)$belongs to $\widehat{S O\left(m_{1}\right)} \times \widehat{S O\left(m_{2}\right)}$ if $m_{1} \equiv m_{2} \equiv 0(\bmod 2)$;

- the element $\operatorname{vol}_{r}$ is mapped to $\pm \operatorname{Id}_{\Delta_{r}^{ \pm}}$, and $\left(\operatorname{Id}_{m_{1}}, \operatorname{Id}_{m_{2}}, \operatorname{vol}_{r}\right)$ maps to $\left(\operatorname{Id}_{m_{1}} \otimes \operatorname{Id}_{\Delta_{r}^{+}} \oplus(-1) \operatorname{Id}_{m_{2}} \otimes\right.$ $\left.\operatorname{Id}_{\Delta_{r}^{-}}\right) \in S O(N)$; the element $\left(\operatorname{Id}_{m_{1}} \otimes \operatorname{Id}_{\Delta_{r}^{+}} \oplus(-1) \operatorname{Id}_{m_{2}} \otimes \operatorname{Id}_{\Delta_{r}^{-}}\right)$belongs to $\left.\left.\widehat{S O\left(m_{1}\right.}\right) \times \widehat{S O\left(m_{2}\right.}\right)$ if $m_{2} \equiv 0(\bmod 2) ;$

- the element $-\mathrm{vol}_{r}$ is mapped to $\mp \mathrm{Id}_{\Delta_{r}^{ \pm}}$, and $\left(\mathrm{Id}_{m_{1}}, \mathrm{Id}_{m_{2}},-\mathrm{vol}_{r}\right)$ maps to $\left((-1) \operatorname{Id}_{m_{1}} \otimes \mathrm{Id}_{\Delta_{r}^{+}} \oplus \operatorname{Id}_{m_{2}} \otimes\right.$ $\left.\operatorname{Id}_{\Delta_{r}^{-}}\right) \in S O(N)$; the element $\left((-1) \operatorname{Id}_{m_{1}} \otimes \operatorname{Id}_{\Delta_{r}^{+}} \oplus \operatorname{Id}_{m_{2}} \otimes \operatorname{Id}_{\Delta_{r}^{-}}\right)$belongs to $\left.\widehat{S O\left(m_{1}\right.}\right) \times \widehat{S O\left(m_{2}\right)}$ if $m_{1} \equiv 0(\bmod 2)$.

Thus,

(1) if $m_{1} \equiv m_{2} \equiv 1(\bmod 2)$,

$$
\begin{aligned}
\operatorname{ker}(\rho) & =\left\{\left(\operatorname{Id}_{m_{1}}, \operatorname{Id}_{m_{2}}, 1\right)\right\}, \\
\left.\left(\widehat{S O\left(m_{1}\right.}\right) \times \widehat{S O\left(m_{2}\right)}\right) \widehat{\operatorname{Spin}(r)} & \cong S O\left(m_{1}\right) \times S O\left(m_{2}\right) \times \operatorname{Spin}(r)
\end{aligned}
$$

(2) if $m_{1} \equiv 0(\bmod 2), m_{2} \equiv 1(\bmod 2)$,

$$
\begin{aligned}
\operatorname{ker}(\rho) & =\left\{\left(\mathrm{Id}_{m_{1}}, \mathrm{Id}_{m_{2}}, 1\right),\left(-\mathrm{Id}_{m_{1}}, \mathrm{Id}_{m_{2}},-\operatorname{vol}_{r}\right)\right\} \cong \mathbb{Z}_{2}, \\
\left(\widehat{S O\left(m_{1}\right)} \times \widehat{S O\left(m_{2}\right)}\right) \widehat{\operatorname{Spin}(r)} & \cong \frac{S O\left(m_{1}\right) \times S O\left(m_{2}\right) \times \operatorname{Spin}(r)}{\mathbb{Z}_{2}}
\end{aligned}
$$

(3) if $m_{1} \equiv 1(\bmod 2), m_{2} \equiv 0(\bmod 2)$,

$$
\begin{aligned}
\operatorname{ker}(\rho) & =\left\{\left(\operatorname{Id}_{m_{1}}, \operatorname{Id}_{m_{2}}, 1\right),\left(\operatorname{Id}_{m_{1}},-\mathrm{Id}_{m_{2}}, \operatorname{vol}\right)\right\} \cong \mathbb{Z}_{2}, \\
\left(\widehat{S O\left(m_{1}\right)} \times \widehat{S O\left(m_{2}\right)}\right) \widehat{\operatorname{Spin}(r)} & \cong \frac{S O\left(m_{1}\right) \times S O\left(m_{2}\right) \times \operatorname{Spin}(r)}{\mathbb{Z}_{2}}
\end{aligned}
$$

(4) if $m_{1} \equiv m_{2} \equiv 0(\bmod 2)$,

$$
\begin{aligned}
& \operatorname{ker}(\rho)=\left\{\left(\mathrm{Id}_{m_{1}}, \mathrm{Id}_{m_{2}}, 1\right),\left(-\mathrm{Id}_{m_{1}},-\mathrm{Id}_{m_{2}},-1\right),\right. \\
&\left.\left(\mathrm{Id}_{m_{1}},-\mathrm{Id}_{m_{2}}, \operatorname{vol}\right),\left(-\mathrm{Id}_{m_{1}}, \mathrm{Id}_{m_{2}},-\operatorname{vol}\right)\right\} \\
& \cong \mathbb{Z}_{2} \oplus \mathbb{Z}_{2}, \\
&\left(\widehat{S O\left(m_{1}\right)} \times \widehat{S O\left(m_{2}\right)}\right) \widehat{\operatorname{Spin}(r)} \cong \frac{S O\left(m_{1}\right) \times S O\left(m_{2}\right) \times \operatorname{Spin}(r)}{\mathbb{Z}_{2} \oplus \mathbb{Z}_{2}} .
\end{aligned}
$$


- Case $m_{1}>0, m_{2}=0$. If there are elements $g_{1} \in S O\left(m_{1}\right)$ and $h \in \operatorname{Spin}(r)$ such that

$$
\rho\left(g_{1}, h\right)=\operatorname{Id}_{N},
$$

then

$$
\widehat{\operatorname{Sin}(r)}+\ni \rho\left(\operatorname{Id}_{m_{1}}, h\right)=\rho\left(g_{1}, 1\right)^{-1} \in \widehat{S O\left(m_{1}\right)} .
$$

Since $\rho\left(\operatorname{Id}_{m_{1}}, h\right)$ commutes with every element of $\widehat{\operatorname{Sin}(r)}+$, it belongs to its center $Z\left(\widehat{\operatorname{Sin}(r)^{+}}\right) \cong$ $Z\left(\operatorname{Spin}(r) /\left\{1, \operatorname{vol}_{r}\right\}\right)=\left\{1,-1, \operatorname{vol}_{r},-\operatorname{vol}_{r}\right\} /\left\{1, \operatorname{vol}_{r}\right\} \cong \mathbb{Z}_{2}$. Note that

- the element -1 is mapped to $-\operatorname{Id}_{\Delta_{r}^{+}}$in the $\operatorname{Spin}(r)$ representation $\Delta_{r}^{+}$, and $\left(\operatorname{Id}_{m_{1}},-1\right)$ maps to $-\operatorname{Id}_{m_{1}} \otimes \operatorname{Id}_{\Delta_{r}^{+}} \in S O(N)$; the element $-\operatorname{Id}_{m_{1}} \otimes \operatorname{Id}_{\Delta_{r}^{+}}$belongs to $\left.\widehat{S O\left(m_{1}\right.}\right)$ if $m_{1} \equiv 0(\bmod 2)$.

Thus,

(5) if $m_{1} \equiv 1(\bmod 2)$,

$$
\begin{aligned}
\operatorname{ker}(\rho) & =\left\{\left(\operatorname{Id}_{m_{1}}, 1\right),\left(\operatorname{Id}_{m_{1}}, \operatorname{vol}_{r}\right)\right\} \\
\widehat{S O\left(m_{1}\right)} \widehat{\operatorname{Sin}(r)}+ & \cong \frac{S O\left(m_{1}\right) \times \operatorname{Spin}(r)}{\mathbb{Z}_{2}}
\end{aligned}
$$

(6) if $m_{1} \equiv 0(\bmod 2)$,

$$
\begin{aligned}
\operatorname{ker}(\rho) & =\left\{\left(\operatorname{Id}_{m_{1}}, 1\right),\left(-\operatorname{Id}_{m_{1}},-1\right),\left(\operatorname{Id}_{m_{1}}, \operatorname{vol}_{r}\right),\left(-\operatorname{Id}_{m_{1}},-\operatorname{vol}_{r}\right)\right\} \\
\widehat{S O\left(m_{1}\right)} \widehat{\operatorname{Sin}(r)}+ & \cong \frac{S O\left(m_{1}\right) \times \operatorname{Spin}(r)}{\mathbb{Z}_{2} \oplus \mathbb{Z}_{2}} .
\end{aligned}
$$

- Case $m_{1}=0, m_{2}>0$. If there are elements $g_{2} \in S O\left(m_{2}\right)$ and $h \in \operatorname{Spin}(r)$ such that

$$
\rho\left(g_{2}, h\right)=\operatorname{Id}_{N},
$$

then

$$
\widehat{\operatorname{Spin}(r)}-\ni \rho\left(\operatorname{Id}_{m_{2}}, h\right)=\rho\left(g_{2}, 1\right)^{-1} \in \widehat{S O\left(m_{2}\right)} .
$$

Since $\rho\left(\operatorname{Id}_{m_{2}}, h\right)$ commutes with every element of $\widehat{\operatorname{Spin}(r)^{-}}-$, it belongs to its center $Z\left(\widehat{\operatorname{Sin}(r)^{-}}\right) \cong$ $Z\left(\operatorname{Spin}(r) /\left\{1,-\operatorname{vol}_{r}\right\}\right)=\left\{1,-1, \operatorname{vol}_{r},-\operatorname{vol}_{r}\right\} /\left\{1,-\operatorname{vol}_{r}\right\} \cong \mathbb{Z}_{2}$. Note that

- the element -1 is mapped to $-\operatorname{Id}_{\Delta_{r}^{-}}$in the $\operatorname{Spin}(r)$ representation $\Delta_{r}^{-}$, and $\left(\operatorname{Id}_{m_{2}},-1\right)$ maps to $-\mathrm{Id}_{m_{2}} \otimes \mathrm{Id}_{\Delta_{r}^{-}} \in S O(N)$; the element $-\mathrm{Id}_{m_{2}} \otimes \mathrm{Id}_{\Delta_{r}^{-}}$belongs to $\widehat{S O\left(m_{2}\right)}$ if $m_{2} \equiv 0(\bmod 2)$;

Thus,

(7) if $m_{2} \equiv 1(\bmod 2)$,

$$
\begin{aligned}
\operatorname{ker}(\rho) & =\left\{\left(\operatorname{Id}_{m_{2}}, 1\right),\left(\operatorname{Id}_{m_{2}},-\operatorname{vol}_{r}\right)\right\} \\
\widehat{S O\left(m_{2}\right)} \widehat{\operatorname{Spin}(r)}- & \cong \frac{S O\left(m_{2}\right) \times \operatorname{Spin}(r)}{\mathbb{Z}_{2}}
\end{aligned}
$$

(8) if $m_{2} \equiv 0(\bmod 2)$,

$$
\begin{aligned}
\operatorname{ker}(\rho) & =\left\{\left(\operatorname{Id}_{m_{2}}, 1\right),\left(-\operatorname{Id}_{m_{2}},-1\right),\left(-\operatorname{Id}_{m_{2}}, \operatorname{vol}_{r}\right),\left(\operatorname{Id}_{m_{2}},-\operatorname{vol}_{r}\right)\right\} \\
\widehat{S O\left(m_{2}\right)} \widehat{\operatorname{Sin}(r)}- & \cong \frac{S O\left(m_{2}\right) \times \operatorname{Spin}(r)}{\mathbb{Z}_{2} \oplus \mathbb{Z}_{2}} .
\end{aligned}
$$

Fundamental group. We will now analyze each of the previous eight cases:

(1) Recall that $m_{1}, m_{2}>0$ and $m_{1} \equiv m_{2} \equiv 1(\bmod 2)$. 
- If $m_{1}, m_{2} \geq 3$, let $\tilde{\rho}$ denote the following map

$$
\begin{gathered}
\operatorname{Spin}\left(m_{1}\right) \times \operatorname{Spin}\left(m_{2}\right) \times \operatorname{Spin}(r) \\
\downarrow \\
S O\left(m_{1}\right) \times S O\left(m_{2}\right) \times \operatorname{Spin}(r)
\end{gathered}
$$

Thus

$$
\begin{aligned}
\operatorname{ker}(\tilde{\rho}) & =\{(1,1,1),(-1,1,1),(1,-1,1),(-1,-1,1)\}, \\
\pi_{1}\left(\left(\widehat{S O\left(m_{1}\right)} \times \widehat{S O\left(m_{2}\right)}\right) \widehat{\operatorname{Sin}(r)}\right) & \cong \mathbb{Z}_{2} \oplus \mathbb{Z}_{2} .
\end{aligned}
$$

- If $m_{1}=1, m_{2} \geq 3, S O\left(m_{1}\right)=\left\{\operatorname{Id}_{1}\right\}$ and let $\tilde{\rho}$ denote the following map

$$
\begin{gathered}
\left\{\operatorname{Id}_{1}\right\} \times \operatorname{Spin}\left(m_{2}\right) \times \operatorname{Spin}(r) \\
\downarrow \\
\left\{\operatorname{Id}_{1}\right\} \times S O\left(m_{2}\right) \times \operatorname{Spin}(r)
\end{gathered}
$$

Thus

$$
\begin{aligned}
\operatorname{ker}(\tilde{\rho}) & =\left\{\left(\operatorname{Id}_{1}, 1,1\right),\left(\operatorname{Id}_{1},-1,1\right)\right\}, \\
\pi_{1}\left(\left(\widehat{\operatorname{SO}(1)} \times \widehat{S O\left(m_{2}\right)}\right) \widehat{\operatorname{Spin}(r)}\right) & \cong \mathbb{Z}_{2} .
\end{aligned}
$$

- If $m_{1} \geq 3, m_{2}=1$, let $\tilde{\rho}$ denote the following map

$$
\begin{gathered}
\operatorname{Spin}\left(m_{1}\right) \times\left\{\operatorname{Id}_{1}\right\} \times \operatorname{Spin}(r) \\
\downarrow \\
S O\left(m_{1}\right) \times\left\{\operatorname{Id}_{1}\right\} \times \operatorname{Spin}(r)
\end{gathered}
$$

Thus

$$
\begin{aligned}
& \operatorname{ker}(\tilde{\rho}) \\
\left.\pi_{1}\left(\left(\widehat{S O\left(m_{1}\right.}\right) \times \widehat{S O(1)}\right) \widehat{\operatorname{Spin}(r)}\right) & \cong \mathbb{Z}_{2} .
\end{aligned}
$$

- If $m_{1}=1, m_{2}=1$, let $\tilde{\rho}$ be

$$
\begin{gathered}
\left\{\operatorname{Id}_{1}\right\} \times\left\{\operatorname{Id}_{1}\right\} \times \operatorname{Spin}(r) \\
\downarrow \\
\left\{\operatorname{Id}_{1}\right\} \times\left\{\operatorname{Id}_{1}\right\} \times \operatorname{Spin}(r)
\end{gathered}
$$

Thus,

$$
\begin{aligned}
\operatorname{ker}(\tilde{\rho}) & =\left\{\left(\operatorname{Id}_{1}, \mathrm{Id}_{1}, 1\right)\right\}, \\
\pi_{1}((\widehat{\operatorname{SO(1)}} \times \widehat{\operatorname{SO(1)}}) \widehat{\operatorname{Spin}(r)}) & \cong\{1\} .
\end{aligned}
$$

(2) Recall that $m_{1}, m_{2}>0, m_{1} \equiv 0(\bmod 2), m_{2} \equiv 1(\bmod 2)$.

- If $m_{1} \geq 4, m_{2} \geq 3$, let $\tilde{\rho}$ denote the composition

$$
\begin{gathered}
\operatorname{Spin}\left(m_{1}\right) \times \operatorname{Spin}\left(m_{2}\right) \times \operatorname{Spin}(r) \\
\downarrow \\
\operatorname{SO}\left(m_{1}\right) \times S O\left(m_{2}\right) \times \operatorname{Spin}(r) \\
\downarrow \\
\frac{S O\left(m_{1}\right) \times S O\left(m_{2}\right) \times \operatorname{Spin}(r)}{\mathbb{Z}_{2}}
\end{gathered}
$$

Thus

$$
\begin{aligned}
\operatorname{ker}(\tilde{\rho}) & = \begin{cases}\left\langle(1,-1,1),(-1,1,1),\left(\operatorname{vol}_{m_{1}}, 1,-\operatorname{vol}_{r}\right)\right\rangle & \text { if } m_{1} \equiv 0(\bmod 4), \\
\left\langle(1,-1,1),\left(\operatorname{vol}_{m_{1}}, 1,-\operatorname{vol}_{r}\right)\right\rangle & \text { if } m_{1} \equiv 2(\bmod 4),\end{cases} \\
\pi_{1}\left(\left(\widehat{S O\left(m_{1}\right)} \times \widehat{S O\left(m_{2}\right)}\right) \widehat{\operatorname{Spin}(r)}\right) & \cong \begin{cases}\mathbb{Z}_{2} \oplus \mathbb{Z}_{2} \oplus \mathbb{Z}_{2} & \text { if } m_{1} \equiv 0(\bmod 4), \\
\mathbb{Z}_{2} \oplus \mathbb{Z}_{4} & \text { if } m_{1} \equiv 2(\bmod 4) .\end{cases}
\end{aligned}
$$


- If $m_{1}=2, m_{2} \geq 3, S O\left(m_{1}\right)=S O(2)$ and let $\tilde{\rho}$ denote the following composition

$$
\begin{gathered}
\mathbb{R} \times \operatorname{Spin}\left(m_{2}\right) \times \operatorname{Spin}(r) \\
\downarrow \\
S O(2) \times S O\left(m_{2}\right) \times \operatorname{Spin}(r) \\
\downarrow \\
\frac{\operatorname{SO}(2) \times S O\left(m_{2}\right) \times \operatorname{Spin}(r)}{\mathbb{Z}_{2}}
\end{gathered}
$$

Thus,

$$
\begin{aligned}
\operatorname{ker}(\tilde{\rho}) & =\left\langle(0,-1,1),\left(\pi, 1,-\operatorname{vol}_{r}\right)\right\rangle, \\
\pi_{1}\left(\left(\widehat{\operatorname{SO}(2)} \times \widehat{S O\left(m_{2}\right)}\right) \widehat{\operatorname{Spin}(r)}\right) & =\mathbb{Z}_{2} \oplus \mathbb{Z} .
\end{aligned}
$$

- If $m_{1} \geq 4, m_{2}=1$, let $\tilde{\rho}$ denote the composition

$$
\begin{gathered}
\operatorname{Spin}\left(m_{1}\right) \times\left\{\operatorname{Id}_{1}\right\} \times \operatorname{Spin}(r) \\
\downarrow \\
S O\left(m_{1}\right) \times\left\{\operatorname{Id}_{1}\right\} \times \operatorname{Spin}(r) \\
\downarrow \\
\frac{S O\left(m_{1}\right) \times\left\{\operatorname{Id}_{1}\right\} \times \operatorname{Spin}(r)}{\mathbb{Z}_{2}}
\end{gathered}
$$

Thus

$$
\begin{aligned}
& \operatorname{ker}(\tilde{\rho})= \begin{cases}\left\langle\left(-1, \mathrm{Id}_{1}, 1\right),\left(\operatorname{vol}_{m_{1}}, \mathrm{Id}_{1},-\operatorname{vol}_{r}\right)\right\rangle & \text { if } m_{1} \equiv 0(\bmod 4), \\
\left\langle\left(\operatorname{vol}_{m_{1}}, \mathrm{Id}_{1},-\operatorname{vol}_{r}\right)\right\rangle & \text { if } m_{1} \equiv 2(\bmod 4),\end{cases} \\
& \pi_{1}\left(\left(\widehat{\left.\operatorname{SO(m_{1}}\right)} \times \widehat{\operatorname{SO(1)})} \widehat{\operatorname{Spin}(r)}\right)= \begin{cases}\mathbb{Z}_{2} \oplus \mathbb{Z}_{2} & \text { if } m_{1} \equiv 0(\bmod 4), \\
\mathbb{Z}_{4} & \text { if } m_{1} \equiv 2(\bmod 4) .\end{cases} \right.
\end{aligned}
$$

- If $m_{1}=2, m_{2}=1$, let $\tilde{\rho}$ denote the composition

$$
\begin{gathered}
\mathbb{R} \times\left\{\operatorname{Id}_{1}\right\} \times \operatorname{Spin}(r) \\
\downarrow \\
S O(2) \times\left\{\operatorname{Id}_{1}\right\} \times \operatorname{Spin}(r) \\
\downarrow \\
\frac{\operatorname{SO}(2) \times\left\{\operatorname{Id}_{1}\right\} \times \operatorname{Spin}(r)}{\mathbb{Z}_{2}}
\end{gathered}
$$

Thus

$$
\begin{aligned}
& \operatorname{ker}(\tilde{\rho})=\left\langle\left(\pi, \operatorname{Id}_{1},-\operatorname{vol}_{r}\right)\right\rangle, \\
& \pi_{1}((\widehat{S O(2)} \times \widehat{S O(1)}) \widehat{\operatorname{Spin}(r)}) \cong \mathbb{Z} .
\end{aligned}
$$

(3) Recall that $m_{1}, m_{2}>0, m_{1} \equiv 1(\bmod 2), m_{2} \equiv 0(\bmod 2)$.

- If $m_{1} \geq 3, m_{2} \geq 4$, let $\tilde{\rho}$ denote the composition

$$
\begin{gathered}
\operatorname{Spin}\left(m_{1}\right) \times \operatorname{Spin}\left(m_{2}\right) \times \operatorname{Spin}(r) \\
\downarrow \\
S O\left(m_{1}\right) \times S O\left(m_{2}\right) \times \operatorname{Spin}(r) \\
\downarrow \\
\frac{S O\left(m_{1}\right) \times S O\left(m_{2}\right) \times \operatorname{Spin}(r)}{\mathbb{Z}_{2}}
\end{gathered}
$$

Thus,

$$
\begin{aligned}
\operatorname{ker}(\tilde{\rho}) & = \begin{cases}\left\langle(-1,1,1),(1,-1,1),\left(1, \operatorname{vol}_{m_{2}}, \operatorname{vol}_{r}\right)\right\rangle & \text { if } m_{2} \equiv 0(\bmod 4), \\
\left\langle(-1,1,1),\left(1, \operatorname{vol}_{m_{2}}, \operatorname{vol}_{r}\right)\right\rangle & \text { if } m_{2} \equiv 2(\bmod 4),\end{cases} \\
\left.\pi_{1}\left(\left(\widehat{S O\left(m_{1}\right.}\right) \times \widehat{S O\left(m_{2}\right)}\right) \widehat{\operatorname{Spin}(r)}\right) & = \begin{cases}\mathbb{Z}_{2} \oplus \mathbb{Z}_{2} \oplus \mathbb{Z}_{2} & \text { if } m_{2} \equiv 0(\bmod 4), \\
\mathbb{Z}_{2} \oplus \mathbb{Z}_{4} & \text { if } m_{2} \equiv 2(\bmod 4) .\end{cases}
\end{aligned}
$$


- If $m_{1} \geq 3, m_{2}=2$, let $\tilde{\rho}$ denote the composition

$$
\begin{aligned}
& \operatorname{Spin}\left(m_{1}\right) \times \mathbb{R} \times \operatorname{Spin}(r) \\
& S O\left(m_{1}\right) \times S O(2) \times \operatorname{Spin}(r) \\
& \frac{\downarrow}{\operatorname{SO}\left(m_{1}\right) \times S O(2) \times \operatorname{Spin}(r)}
\end{aligned}
$$

Thus,

$$
\begin{aligned}
&\left.\pi_{1}\left(\left(\widehat{\operatorname{SOr}\left(m_{1}\right)}\right) \times \widehat{\operatorname{SO}(2)}\right) \widehat{\operatorname{Spin}(r)}\right) \cong\left\langle(-1,0,1),\left(1, \pi, \operatorname{vol}_{r}\right)\right\rangle, \\
& \mathbb{Z}_{2} \oplus \mathbb{Z} .
\end{aligned}
$$

- If $m_{1}=1, m_{2} \geq 4$, let $\tilde{\rho}$ denote the composition

$$
\begin{gathered}
\left\{\operatorname{Id}_{1}\right\} \times \operatorname{Spin}\left(m_{2}\right) \times \operatorname{Spin}(r) \\
\downarrow \\
\left\{\operatorname{Id}_{1}\right\} \times S O\left(m_{2}\right) \times \operatorname{Spin}(r) \\
\downarrow \\
\frac{\left\{\operatorname{Id}_{1}\right\} \times S O\left(m_{2}\right) \times \operatorname{Spin}(r)}{\mathbb{Z}_{2}}
\end{gathered}
$$

Thus,

$$
\begin{aligned}
& \operatorname{ker}(\tilde{\rho})= \begin{cases}\left\langle\left(\mathrm{Id}_{1},-1,1\right),\left(\mathrm{Id}_{1}, \operatorname{vol}_{m_{2}}, \operatorname{vol}_{r}\right)\right\rangle & \text { if } m_{2} \equiv 0(\bmod 4), \\
\left\langle\left(\operatorname{Id}_{1}, \operatorname{vol}_{m_{2}}, \operatorname{vol}_{r}\right)\right\rangle & \text { if } m_{2} \equiv 2(\bmod 4),\end{cases} \\
& \pi_{1}\left(\left(\widehat{S O\left(m_{1}\right)} \times \widehat{S O\left(m_{2}\right)}\right) \widehat{\operatorname{Spin}(r)}\right)= \begin{cases}\mathbb{Z}_{2} \oplus \mathbb{Z}_{2} & \text { if } m_{2} \equiv 0(\bmod 4), \\
\mathbb{Z}_{4} & \text { if } m_{2} \equiv 2(\bmod 4) .\end{cases}
\end{aligned}
$$

- If $m_{1}=1, m_{2}=2$, let $\tilde{\rho}$ denote the composition

$$
\begin{gathered}
\left\{\operatorname{Id}_{1}\right\} \times \mathbb{R} \times \operatorname{Spin}(r) \\
\downarrow \\
\left\{\operatorname{Id}_{1}\right\} \times S O(2) \times \operatorname{Spin}(r) \\
\downarrow \\
\frac{\left\{\operatorname{Id}_{1}\right\} \times S O(2) \times \operatorname{Spin}(r)}{\mathbb{Z}_{2}}
\end{gathered}
$$

Thus,

$$
\begin{aligned}
\operatorname{ker}(\tilde{\rho}) & =\left\langle\left(\operatorname{Id}_{1}, \pi, \operatorname{vol}_{r}\right)\right\rangle, \\
\pi_{1}((\widehat{S O(1)} \times \widehat{S O(2)}) \widehat{\operatorname{Spin}(r)}) & \cong \mathbb{Z} .
\end{aligned}
$$

(4) Recall that $m_{1}, m_{2}>0, m_{1} \equiv m_{2} \equiv 0(\bmod 2)$.

- If $m_{1}, m_{2} \geq 4$, let $\tilde{\rho}$ denote the composition

$$
\begin{gathered}
\operatorname{Spin}\left(m_{1}\right) \times \operatorname{Spin}\left(m_{2}\right) \times \operatorname{Spin}(r) \\
\downarrow \\
\operatorname{SO}\left(m_{1}\right) \times S O\left(m_{2}\right) \times \operatorname{Spin}(r) \\
\downarrow \\
\frac{S O\left(m_{1}\right) \times S O\left(m_{2}\right) \times \operatorname{Spin}(r)}{\mathbb{Z}_{2} \oplus \mathbb{Z}_{2}}
\end{gathered}
$$

Thus,

$$
\begin{aligned}
& \operatorname{ker}(\tilde{\rho})=\left\{\begin{array}{l}
\left\langle(-1,1,1),(1,-1,1),\left(1, \operatorname{vol}_{m_{2}}, \operatorname{vol}_{r}\right),\left(\operatorname{vol}_{m_{1}}, 1,-\operatorname{vol}_{r}\right)\right\rangle \\
\left.(-1,1,1),\left(\operatorname{vol}_{m_{1}}, 1,-\operatorname{vol}_{r}\right),\left(1, \operatorname{vol}_{m_{2}}, \operatorname{vol}_{r}\right)\right\rangle \\
\left.(1,-1,1),\left(\operatorname{vol}_{m_{1}}, 1,-\operatorname{vol}_{r}\right),\left(1, \operatorname{vol}_{m_{2}}, \operatorname{vol}_{r}\right)\right\rangle \\
\left.\left(\operatorname{vol}_{m_{1}}, 1,-\operatorname{vol}_{r}\right),\left(1, \operatorname{vol}_{m_{2}}, \operatorname{vol}_{r}\right)\right\rangle
\end{array}\right. \\
& \text { if } m_{1} \equiv m_{2} \equiv 0(\bmod 4) \\
& \text { if } m_{1} \equiv 0(\bmod 4) \text { and } m_{2} \equiv 2(\bmod 4) \text {, } \\
& \text { if } m_{2} \equiv 2(\bmod 4) \text { and } m_{2} \equiv 0(\bmod 4) \text {, } \\
& \text { if } m_{1} \equiv m_{2} \equiv 2(\bmod 4) \text {, } \\
& \left.\pi_{1}\left(\left(S \widehat{O\left(m_{1}\right.}\right) \times S \widehat{O\left(m_{2}\right)}\right) \widehat{\sin (r)}\right) \cong \begin{cases}\mathbb{Z}_{2} \oplus \mathbb{Z}_{2} \oplus \mathbb{Z}_{2} \oplus \mathbb{Z}_{2} & \text { if } m_{1} \equiv m_{2} \equiv 0(\bmod 4), \\
\mathbb{Z}_{2} \oplus \mathbb{Z}_{2} \oplus \mathbb{Z}_{4} & \text { if } m_{1} \equiv 0(\bmod 4) \text { and } m_{2} \equiv 2(\bmod 4), \\
\mathbb{Z}_{2} \oplus \mathbb{Z}_{4} \oplus \mathbb{Z}_{2} & \text { if } m_{2} \equiv 2(\bmod 4) \text { and } m_{2} \equiv 0(\bmod 4), \\
\mathbb{Z}_{4} \oplus \mathbb{Z}_{4} & \text { if } m_{1} \equiv m_{2} \equiv 2(\bmod 4) .\end{cases}
\end{aligned}
$$


- If $m_{1} \geq 4, m_{2}=2$, let $\tilde{\rho}$ denote the composition

$$
\begin{gathered}
\operatorname{Spin}\left(m_{1}\right) \times \mathbb{R} \times \operatorname{Spin}(r) \\
\downarrow \\
\operatorname{SO}\left(m_{1}\right) \times S O(2) \times \operatorname{Spin}(r) \\
\downarrow \\
\frac{\operatorname{SO}\left(m_{1}\right) \times S O(2) \times \operatorname{Spin}(r)}{\mathbb{Z}_{2} \oplus \mathbb{Z}_{2}}
\end{gathered}
$$

Thus,

$$
\begin{aligned}
& \operatorname{ker}(\tilde{\rho})= \begin{cases}\left\langle(-1,0,1),\left(\operatorname{vol}_{m_{1}}, 0,-\operatorname{vol}_{r}\right),\left(1, \pi, \operatorname{vol}_{r}\right)\right\rangle & \text { if } m_{1} \equiv 0(\bmod 4), \\
\left\langle\left(\operatorname{vol}_{m_{1}}, 0,-\operatorname{vol}_{r}\right),\left(1, \pi, \operatorname{vol}_{r}\right)\right\rangle & \text { if } m_{1} \equiv 2(\bmod 4),\end{cases} \\
& \pi_{1}\left(\left(\widehat{S O\left(m_{1}\right)} \times \widehat{S O(2)}\right) \widehat{\operatorname{Spin}(r)}\right) \cong \begin{cases}\mathbb{Z}_{2} \oplus \mathbb{Z}_{2} \oplus \mathbb{Z} & \text { if } m_{1} \equiv 0(\bmod 4), \\
\mathbb{Z}_{4} \oplus \mathbb{Z} & \text { if } m_{1} \equiv 2(\bmod 4) .\end{cases}
\end{aligned}
$$

- If $m_{1}=2, m_{2} \geq 4$, let $\tilde{\rho}$ denote the composition

$$
\begin{gathered}
\mathbb{R} \times \operatorname{Spin}\left(m_{2}\right) \times \operatorname{Spin}(r) \\
\downarrow \\
\qquad \begin{array}{c}
\downarrow \\
S O
\end{array}(2) \times S O\left(m_{2}\right) \times \operatorname{Spin}(r) \\
\downarrow \\
\frac{\operatorname{SO}(2) \times S O\left(m_{2}\right) \times \operatorname{Spin}(r)}{\mathbb{Z}_{2} \oplus \mathbb{Z}_{2}}
\end{gathered}
$$

Thus,

$$
\begin{aligned}
& \operatorname{ker}(\tilde{\rho})= \begin{cases}\left\langle(0,-1,1),\left(0, \operatorname{vol}_{m_{2}}, \operatorname{vol}_{r}\right),\left(\pi, 1,-\operatorname{vol}_{r}\right)\right\rangle & \text { if } m_{2} \equiv 0(\bmod 4), \\
\left\langle\left(0, \operatorname{vol}_{m_{2}}, \operatorname{vol}_{r}\right),\left(\pi, 1,-\operatorname{vol}_{r}\right)\right\rangle & \text { if } m_{2} \equiv 2(\bmod 4),\end{cases} \\
& \pi_{1}\left(\left(\widehat{\operatorname{SO}(2)} \times \widehat{S O\left(m_{2}\right)}\right) \widehat{\operatorname{Spin}(r)}\right) \cong \begin{cases}\mathbb{Z}_{2} \oplus \mathbb{Z}_{2} \oplus \mathbb{Z} & \text { if } m_{2} \equiv 0(\bmod 4), \\
\mathbb{Z}_{4} \oplus \mathbb{Z} & \text { if } m_{2} \equiv 2(\bmod 4) .\end{cases}
\end{aligned}
$$

- If $m_{1}=m_{2}=2$, let $\tilde{\rho}$ denote the composition

$$
\begin{gathered}
\mathbb{R} \times \mathbb{R} \times \operatorname{Spin}(r) \\
\downarrow \\
S O(2) \times S O(2) \times \operatorname{Spin}(r) \\
\downarrow \\
\frac{S O(2) \times S O(2) \times \operatorname{Spin}(r)}{\mathbb{Z}_{2} \oplus \mathbb{Z}_{2}}
\end{gathered}
$$

Thus,

$$
\begin{aligned}
\operatorname{ker}(\tilde{\rho}) & =\left\langle\left(0, \pi, \operatorname{vol}_{r}\right),\left(\pi, 0,-\operatorname{vol}_{r}\right)\right\rangle, \\
\pi_{1}((\widehat{S O(2)} \times \widehat{S O(2)}) \widehat{\operatorname{Spin}(r)}) & \cong \mathbb{Z} \oplus \mathbb{Z} .
\end{aligned}
$$

(5) Recall that $m_{1}>0, m_{2}=0, m_{1} \equiv 1(\bmod 2)$.

- If $m_{1} \geq 3$, let $\tilde{\rho}$ denote the composition

$$
\begin{gathered}
\operatorname{Spin}\left(m_{1}\right) \times \operatorname{Spin}(r) \\
\downarrow \\
\quad \downarrow \\
\operatorname{SO}\left(m_{1}\right) \times \operatorname{Spin}(r) \\
\downarrow \\
\frac{\operatorname{SO}\left(m_{1}\right) \times \operatorname{Spin}(r)}{\mathbb{Z}_{2}}
\end{gathered}
$$

Thus

$$
\begin{aligned}
\operatorname{ker}(\tilde{\rho}) & =\left\langle(-1,1),\left(1, \operatorname{vol}_{r}\right)\right\rangle \\
\left.\pi_{1}\left(\widehat{S O\left(m_{1}\right)}\right) \widehat{\operatorname{pin}(r)}+\right) & \cong \mathbb{Z}_{2} \oplus \mathbb{Z}_{2} .
\end{aligned}
$$


- If $m_{1}=1$, let $\tilde{\rho}$ denote

$$
\begin{gathered}
\left\{\mathrm{Id}_{1}\right\} \times \operatorname{Spin}(r) \\
\downarrow \\
\frac{\downarrow}{\left\{\mathrm{Id}_{1}\right\} \times \operatorname{Spin}(r)} \\
\mathbb{Z}_{2}
\end{gathered}
$$

Thus,

$$
\begin{aligned}
\operatorname{ker}(\tilde{\rho}) & =\left\langle\left(\operatorname{Id}_{1}, \operatorname{vol}_{r}\right)\right\rangle, \\
\pi_{1}(\widehat{\operatorname{SO(1)} S \widehat{\operatorname{pin}(r)}+}) & \cong \mathbb{Z}_{2} .
\end{aligned}
$$

(6) Recall that $m_{1}>0, m_{2}=0, m_{1} \equiv 0(\bmod 2)$.

- If $m_{1} \geq 4$, let $\tilde{\rho}$ denote the composition

$$
\begin{aligned}
& \operatorname{Spin}\left(m_{1}\right) \times \operatorname{Spin}(r) \\
& \downarrow \\
& \operatorname{SO}\left(m_{1}\right) \times \operatorname{Spin}(r) \\
& \downarrow \\
& \frac{\operatorname{SO}\left(m_{1}\right) \times \operatorname{Spin}(r)}{\mathbb{Z}_{2} \oplus \mathbb{Z}_{2}}
\end{aligned}
$$

Thus,

$$
\begin{aligned}
& \operatorname{ker}(\tilde{\rho})= \begin{cases}\left\langle(-1,1),\left(\operatorname{vol}_{m_{1}},-1\right),\left(1, \operatorname{vol}_{r}\right)\right\rangle & \text { if } m_{1} \equiv 0(\bmod 4), \\
\left\langle\left(\operatorname{vol}_{m_{1}},-1\right),\left(1, \operatorname{vol}_{r}\right)\right\rangle & \text { if } m_{1} \equiv 2(\bmod 4),\end{cases} \\
& \pi_{1}\left(\widehat{\left.\operatorname{SO(m_{1}}\right)} \widehat{\operatorname{Spin}(r)}+\right) \cong \begin{cases}\mathbb{Z}_{2} \oplus \mathbb{Z}_{2} \oplus \mathbb{Z}_{2} & \text { if } m_{1} \equiv 0(\bmod 4), \\
\mathbb{Z}_{2} \oplus \mathbb{Z}_{4} & \text { if } m_{1} \equiv 2(\bmod 4) .\end{cases}
\end{aligned}
$$

- If $m_{1}=2$, let $\tilde{\rho}$ denote the composition

$$
\begin{gathered}
\mathbb{R} \times \operatorname{Spin}(r) \\
\downarrow \\
\operatorname{SO}(2) \times \operatorname{Spin}(r) \\
\downarrow \\
\frac{\operatorname{SO}(2) \times \operatorname{Spin}(r)}{\mathbb{Z}_{2} \oplus \mathbb{Z}_{2}}
\end{gathered}
$$

Thus,

$$
\begin{aligned}
\operatorname{ker}(\tilde{\rho}) & =\left\langle(\pi,-1),\left(0, \operatorname{vol}_{r}\right)\right\rangle, \\
\pi_{1}(\widehat{\operatorname{SO}(2)} \widehat{\operatorname{Sin}(r)}+) & \cong \mathbb{Z} \oplus \mathbb{Z}_{2} .
\end{aligned}
$$

(7) Recall that $m_{1}=0, m_{2}>0, m_{2} \equiv 1(\bmod 2)$.

- If $m_{2} \geq 3$, let $\tilde{\rho}$ denote the composition

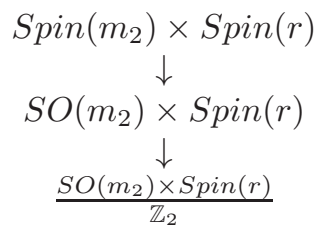

Thus,

$$
\begin{aligned}
\operatorname{ker}(\tilde{\rho}) & =\left\langle\left(1,-\operatorname{vol}_{r}\right)\right\rangle, \\
\pi_{1}(\widehat{\operatorname{SO(1)} S \widehat{\operatorname{pin}(r)}}-) & \cong \mathbb{Z}_{2} .
\end{aligned}
$$


(8) Recall that $m_{1}=0, m_{2}>0, m_{2} \equiv 0(\bmod 2)$.

- If $m_{2} \geq 4$, let $\tilde{\rho}$ denote the composition

$$
\begin{aligned}
& \operatorname{Spin}\left(m_{2}\right) \times \operatorname{Spin}(r) \\
& \downarrow \\
& S O\left(m_{2}\right) \times \operatorname{Spin}(r) \\
& \downarrow \\
& \frac{S O\left(m_{2}\right) \times \operatorname{Spin}(r)}{\mathbb{Z}_{2} \oplus \mathbb{Z}_{2}}
\end{aligned}
$$

Thus,

$$
\begin{aligned}
& \operatorname{ker}(\tilde{\rho})= \begin{cases}\left\langle(-1,1),\left(\operatorname{vol}_{m_{2}},-1\right),\left(1,-\operatorname{vol}_{r}\right)\right\rangle & \text { if } m_{2} \equiv 0(\bmod 4), \\
\left\langle\left(\operatorname{vol}_{m_{2}},-1\right),\left(1,-\operatorname{vol}_{r}\right)\right\rangle & \text { if } m_{2} \equiv 2(\bmod 4),\end{cases} \\
& \pi_{1}\left(\widehat{\left.\operatorname{SO(m_{2}}\right) \operatorname{Spin(r)}}-\right) \cong \begin{cases}\mathbb{Z}_{2} \oplus \mathbb{Z}_{2} \oplus \mathbb{Z}_{2} & \text { if } m_{2} \equiv 0(\bmod 4), \\
\mathbb{Z}_{2} \oplus \mathbb{Z}_{4} & \text { if } m_{2} \equiv 2(\bmod 4) .\end{cases}
\end{aligned}
$$

- If $m_{2}=2$, let $\tilde{\rho}$ denote the composition

$$
\begin{gathered}
\mathbb{R} \times \operatorname{Spin}(r) \\
\downarrow \\
S O(2) \times \operatorname{Spin}(r) \\
\downarrow \\
\frac{\operatorname{SO(2)\times S\operatorname {Spin}(r)}}{\mathbb{Z}_{2} \oplus \mathbb{Z}_{2}}
\end{gathered}
$$

Thus,

$$
\begin{aligned}
\operatorname{ker}(\tilde{\rho}) & =\left\langle(\pi,-1),\left(0,-\operatorname{vol}_{r}\right)\right\rangle, \\
\pi_{1}(\widehat{S O(2)} \widehat{\operatorname{Sin}(r)}-) & \cong \mathbb{Z} \oplus \mathbb{Z}_{2} .
\end{aligned}
$$

\section{$3.3 r \equiv 2,6(\bmod 8)$}

Complexification. The volume form $\operatorname{vol}_{r}=e_{1} \cdots e_{r}$ acts as a complex structure $J$ on $\tilde{\Delta}_{r}$ (cf. [2]). Therefore

$$
\tilde{\Delta}_{r} \otimes \mathbb{C}=\left\{\psi-i J \psi \mid \psi \in \tilde{\Delta}_{r}\right\} \oplus\left\{\psi+i J \psi \mid \psi \in \tilde{\Delta}_{r}\right\} .
$$

Note that the involution $(-i)^{r / 2} \operatorname{vol}_{r}$. acts on $\tilde{\Delta}_{r} \otimes \mathbb{C}$ as follows:

- if $r \equiv 2(\bmod 8)$,

$$
\begin{aligned}
& (-i)^{r / 2} \operatorname{vol}_{r} \cdot(\psi-i J \psi)=(\psi-i J \psi) \\
& (-i)^{r / 2} \operatorname{vol}_{r} \cdot(\psi+i J \psi)=-(\psi+i J \psi)
\end{aligned}
$$

i.e.

$$
\begin{aligned}
\tilde{\Delta}_{r} \otimes \mathbb{C} & =\left\{\psi-i J \psi \mid \psi \in \tilde{\Delta}_{r}\right\} \oplus\left\{\psi+i J \psi \mid \psi \in \tilde{\Delta}_{r}\right\} \\
& =\Delta_{r}^{+} \oplus \Delta_{r}^{-} .
\end{aligned}
$$

- if $r \equiv 6(\bmod 8)$,

$$
\begin{aligned}
& (-i)^{r / 2} \operatorname{vol}_{r} \cdot(\psi-i J \psi)=-(\psi-i J \psi) \\
& (-i)^{r / 2} \operatorname{vol}_{r} \cdot(\psi+i J \psi)=(\psi+i J \psi)
\end{aligned}
$$

i.e.

$$
\begin{aligned}
\tilde{\Delta}_{r} \otimes \mathbb{C} & =\left\{\psi-i J \psi \mid \psi \in \tilde{\Delta}_{r}\right\} \oplus\left\{\psi+i J \psi \mid \psi \in \tilde{\Delta}_{r}\right\} \\
& =\Delta_{r}^{-} \oplus \Delta_{r}^{+} .
\end{aligned}
$$


In any case,

$$
\tilde{\Delta}_{r} \otimes \mathbb{C}=\Delta_{r} .
$$

The centralizer subalgebra of $\widehat{\mathfrak{s p i n}(r)}$ in $\mathfrak{s o}(N)$ is

$$
C_{\mathfrak{s o}(N)}(\widehat{\mathfrak{s p i n}(r)})=\widehat{\mathfrak{u}(m)}=\mathfrak{s o}(m) \otimes \operatorname{Id}_{\tilde{\Delta}_{r}} \oplus S^{2} \mathbb{R}^{m} \otimes J,
$$

where $N=d_{r} m, \mathfrak{s o}(m)$ and $S^{2} \mathbb{R}^{m}$ act on $\mathbb{R}^{m}$ as skew-symmetric and symmetric endomorphisms respectively. Let

$$
v \otimes(\psi-i J \psi) \in\left(\mathbb{R}^{m} \otimes \tilde{\Delta}_{r}\right) \otimes \mathbb{C}
$$

and

$$
A \otimes \operatorname{Id}_{\tilde{\Delta}_{r}}+B \otimes J \in \mathfrak{s o}(m) \otimes \operatorname{Id}_{\tilde{\Delta}_{r}} \oplus S^{2} \mathbb{R}^{m} \otimes J .
$$

Now,

$$
\begin{aligned}
\left(A \otimes \operatorname{Id}_{\tilde{\Delta}_{r}}+B \otimes J\right)(v \otimes(\psi-i J \psi)) & =A v \otimes \psi-i A v \otimes J \psi+B v \otimes J \psi-i B v \otimes J J \psi \\
& =A v \otimes \psi+i B v \otimes \psi+(-i)(i) B v \otimes J \psi-i A v \otimes J \psi \\
& =(A+i B) v \otimes(\psi-i J \psi),
\end{aligned}
$$

where $A+i B \in \mathfrak{u}(m)$. Similarly, for $v \otimes(\psi+i J \psi)$,

$$
\left(A \otimes \operatorname{Id}_{\tilde{\Delta}_{r}}+B \otimes J\right)(v \otimes(\psi+i J \psi))=(A-i B) v \otimes(\psi+i J \psi) .
$$

Thus,

$$
\left(\mathbb{R}^{m} \otimes \tilde{\Delta}_{r}\right) \otimes \mathbb{C}= \begin{cases}\mathbb{C}^{m} \otimes \Delta_{r}^{+} \oplus \overline{\mathbb{C}^{m}} \otimes \Delta_{r}^{-} & \text {if } r \equiv 2(\bmod 8), \\ \mathbb{C}^{m} \otimes \Delta_{r}^{-} \oplus \overline{\mathbb{C}^{m}} \otimes \Delta_{r}^{+} & \text {if } r \equiv 6(\bmod 8),\end{cases}
$$

where $\mathbb{C}^{m}$ is the standard representation of $U(m)$. Therefore, we have a representation

$$
U(m) \times \operatorname{Spin}(r) \longrightarrow S O(N) \subset \operatorname{Aut}\left(\left(\mathbb{R}^{m} \otimes \tilde{\Delta}_{r}\right) \otimes \mathbb{C}\right) .
$$

Structure group. Since $\widehat{\mathfrak{u}(m)}$ and $\widehat{\mathfrak{s p i n}(r)}$ commute with each other, we can take separately the exponentials

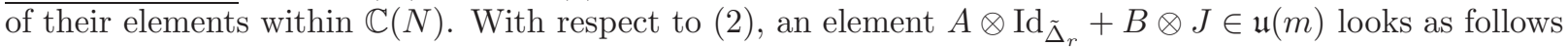

$$
\left(\begin{array}{cc}
(A+i B) \otimes \operatorname{Id}_{\Delta_{r}^{ \pm}} & (A-i B) \otimes \operatorname{Id}_{\Delta_{r}^{\mp}}
\end{array}\right)
$$

so that the exponentials form

$$
\left\{\left(\begin{array}{cc}
e^{A+i B} \otimes \operatorname{Id}_{\Delta_{r}^{ \pm}} & \\
& e^{A-i B} \otimes \operatorname{Id}_{\Delta_{r}^{\mp}}
\end{array}\right): A \in \mathfrak{s o}(m), B \in S^{2} \mathbb{R}^{m}\right\}=: \widehat{U(m)} .
$$

With respect to (2), an element $\operatorname{Id}_{m} \otimes \xi \in \widehat{\mathfrak{s p i n}(r)}$, looks as follows

$$
\left(\begin{array}{cc}
\operatorname{Id}_{m} \otimes \kappa_{r^{*}}^{+}(\xi) & \\
& \operatorname{Id}_{m} \otimes \kappa_{r^{*}}^{-}(\xi)
\end{array}\right)=\operatorname{Id}_{m} \otimes \xi,
$$

and its exponential is

$$
\operatorname{Id}_{m} \otimes e^{\xi} \in \operatorname{Id}_{m} \otimes \kappa(\operatorname{Spin}(r))=: \widehat{\operatorname{Spin}(r)} \cong \operatorname{Spin}(r),
$$

since $\operatorname{Spin}(r)$ is represented faithfully on $\Delta_{r}$. The image of $U(m) \times \operatorname{Spin}(r)$ in $S O(N) \subset \operatorname{Aut}\left(\left(\mathbb{R}^{m} \otimes \tilde{\Delta}_{r}\right) \otimes \mathbb{C}\right)$ under the aforementioned representation is

$$
\mathcal{N}_{S O(N)}^{0}(\widehat{\operatorname{Spin}(r)})=\widehat{U(m)} \widehat{\operatorname{Spin}(r)},
$$


the subgroup of all possible products of elements of the two subgroups, i.e. we have a map

$$
U(m) \times \operatorname{Spin}(r) \stackrel{\rho}{\rightarrow} \widehat{U(m)} \widehat{\operatorname{Spin}(r)} \subset S O(N) .
$$

Now we need to find $\operatorname{ker}(\rho)$ and identify $\widehat{U(m)} \widehat{\operatorname{Spin}(r)}$ as a quotient

$$
\widehat{U(m)} \widehat{\operatorname{Spin}(r)} \cong \frac{U(m) \times \operatorname{Spin}(r)}{\operatorname{ker}(\rho)} .
$$

If there are elements $g \in U(m)$ and $h \in \operatorname{Spin}(r)$ such that

$$
\rho(g, h)=\operatorname{Id}_{N},
$$

then

$$
\widehat{\operatorname{Spin}(r)} \ni \rho\left(\operatorname{Id}_{m}, h\right)=\rho(g, 1)^{-1} \in \widehat{U(m)} .
$$

Since $\rho\left(\operatorname{Id}_{m}, h\right)$ commutes with every element of $\left.\widehat{\operatorname{Sin}(r}\right)$, it belongs to the center $Z(\widehat{\operatorname{Spin}(r)}) \cong Z(\operatorname{Spin}(r))=$ $\left\{1,-1, \operatorname{vol}_{r},-\operatorname{vol}_{r}\right\}=\left\langle\operatorname{vol}_{r}\right\rangle \cong \mathbb{Z}_{4}$. Recall that $\operatorname{vol}_{r}=e_{1} \cdots e_{r}$ acts as $\mp i$ on $\Delta_{r}^{ \pm}$if $r \equiv 2(\bmod 8)$, and as $\pm i$ on $\Delta_{r}^{ \pm}$if $r \equiv 6(\bmod 8)$, so that it maps to

$$
\mp\left(i \operatorname{Id}_{\Delta_{r}^{+}} \oplus(-i) \operatorname{Id}_{\Delta_{r}^{-}}\right)
$$

in the complex $\operatorname{Spin}(r)$ representation. Note that $\left(\mathrm{Id}_{m}, \mathrm{vol}_{r}\right)$ maps to

$$
\begin{array}{ll}
(-i) \operatorname{Id}_{m} \otimes \operatorname{Id}_{\Delta_{r}^{+}} \oplus(i) \operatorname{Id}_{m} \otimes \operatorname{Id}_{\Delta_{r}^{-}} & \text {if } r \equiv 2(\bmod 8), \\
(i) \operatorname{Id}_{m} \otimes \operatorname{Id}_{\Delta_{r}^{+}} \oplus(-i) \operatorname{Id}_{m} \otimes \operatorname{Id}_{\Delta_{r}^{-}} & \text {if } r \equiv 6(\bmod 8),
\end{array}
$$

in $S O(N)$, and that $\left((-i) \operatorname{Id}_{\mathbb{C}^{m}}, 1\right) \in U(m) \times S p i n(r)$ maps to such transformations in both cases. Thus, the elements of $U(m) \times \operatorname{Spin}(r)$ mapping to $\operatorname{Id}_{N}$ are

$$
\pm\left(\operatorname{Id}_{m}, 1\right), \quad \pm\left(i \operatorname{Id}_{m},-\operatorname{vol}_{r}\right),
$$

which form a copy of $\mathbb{Z}_{4}$ and

$$
\widehat{U(m)} \widehat{\operatorname{Spin}(r)} \cong \frac{U(m) \times \operatorname{Spin}(r)}{\mathbb{Z}_{4}} .
$$

Fundamental group. Let

$$
\begin{aligned}
\mathbb{R} \times S U(m) \times \operatorname{Spin}(r) & \stackrel{\tilde{\rho}}{\rightarrow} \widehat{U(m)} \widehat{\operatorname{Spin}(r)} \\
(t, A, g) & \mapsto\left(\begin{array}{cc}
e^{i t} A \otimes \kappa_{r}^{ \pm}(g) & \\
& e^{-i t} \bar{A} \otimes \kappa_{r}^{\mp}(g)
\end{array}\right),
\end{aligned}
$$

Thus

$$
\begin{aligned}
& \operatorname{ker}(\tilde{\rho})=\left\langle\left(\frac{2 \pi}{m}, e^{-\frac{2 \pi i}{m}} \operatorname{Id}_{m}, 1\right),\left(\frac{\pi}{2}, \operatorname{Id}_{m},-\operatorname{vol}\right)\right\rangle, \\
& \pi_{1}\left(\widehat { U ( m ) ( \operatorname { S p i n } ( r ) ) ) } \cong \left\{\begin{array}{ll}
\mathbb{Z}, & \text { if }(m, 4)=1, \\
\mathbb{Z} \oplus \mathbb{Z}_{2}, & \text { if }(m, 4)=2, \\
\mathbb{Z} \oplus \mathbb{Z}_{4}, & \text { if }(m, 4)=4 .
\end{array}\right.\right.
\end{aligned}
$$

Indeed, let

$$
a:=\left(\frac{2 \pi}{m}, e^{-\frac{2 \pi i}{m}} \operatorname{Id}_{m}, 1\right), \quad b:=\left(\frac{\pi}{2}, \operatorname{Id}_{m},-\mathrm{vol}\right),
$$

and note that (in multiplicative notation)

$$
a^{m}=b^{4} .
$$

Moreover, 
- If $(m, 4)=1$, there exist $t, m \in \mathbb{Z}$ coprime such that

$$
t m+s 4=1 .
$$

The element

$$
b^{t} a^{s}
$$

is such that

$$
\begin{aligned}
\left(b^{t} a^{s}\right)^{m} & =b^{m t}\left(b^{4}\right)^{s} \\
& =b, \\
\left(b^{t} a^{s}\right)^{4} & =\left(a^{m}\right)^{t} a^{4 s} \\
& =a .
\end{aligned}
$$

- If $(m, 4)=2, m=4 k+2$ and there exist two generators

$$
\begin{aligned}
c & =a^{-(2 k+1)} b^{2}, \\
d & =b a^{-k},
\end{aligned}
$$

such that

$$
\begin{aligned}
c^{2} & =1, \\
a & =d^{2} c, \\
b & =d^{2 k+1} c^{k} .
\end{aligned}
$$

- If $(m, 4)=4, m=4 k$ and we have two generators

$$
a \text { and } c=a^{-k} b \text {, }
$$

such that

$$
c^{4}=1
$$

\section{$3.4 r \equiv 3,5(\bmod 8)$}

Complexification. In this case $\tilde{\Delta}_{r}$ admits three complex structures $I, J, K$, described explicitly in [2], which behave like quaternions and commute with $\mathfrak{s p i n}(r)$. Let us consider the complexification of $\tilde{\Delta}_{r}$ and decompose as follows

$$
\tilde{\Delta}_{r} \otimes \mathbb{C}=\left\{\psi-i I \psi \mid \psi \in \tilde{\Delta}_{r}\right\} \oplus\left\{\psi+i I \psi \mid \psi \in \tilde{\Delta}_{r}\right\},
$$

where the first and second subspaces are the $+i$ and $-i$ eigenspaces of $I$ respectively. Notice that

$$
\begin{aligned}
J(\psi \mp i I \psi) & =J \psi \mp i J I \psi \\
& =J \psi \pm i I J \psi
\end{aligned}
$$

i.e. $J$ interchanges the two subspaces and squares to $-\operatorname{Id}_{d_{r}}$. For any $\xi \in \mathfrak{s p i n}(r)$

$$
\begin{aligned}
\xi(\psi \pm i I \psi) & =\xi \psi \pm i \xi I \psi \\
& =\xi \psi \pm i I \xi \psi
\end{aligned}
$$

which means that the subspaces $\left\{\psi-i I \psi \mid \psi \in \tilde{\Delta}_{r}\right\}$ and $\left\{\psi+i I \psi \mid \psi \in \tilde{\Delta}_{r}\right\}$ are irreducible complex representations of $\mathfrak{s p i n}(r)$ of dimension $d_{r} / 2$. Thus, they are isomorphic to $\Delta_{r}$ as $\mathfrak{s p i n}(r)$ representations and

$$
\tilde{\Delta}_{r} \otimes \mathbb{C} \cong \Delta_{r} \oplus \Delta_{r}
$$


Now recall that the centralizer subalgebra of $\widehat{\operatorname{spin}(r)}$ is

$$
\begin{aligned}
C_{\mathfrak{s o}(N)}(\widehat{\mathfrak{s p i n}(r)}) & =\mathfrak{s o}(m) \otimes \operatorname{Id}_{\tilde{\Delta}_{r}} \oplus S^{2} \mathbb{R}^{m} \otimes I \oplus S^{2} \mathbb{R}^{m} \otimes J \oplus S^{2} \mathbb{R}^{m} \otimes K \\
& \cong \mathfrak{s o}(m) \otimes \operatorname{Id}_{\tilde{\Delta}_{r}} \oplus S^{2} \mathbb{R}^{m} \otimes \mathfrak{s p}(1) \\
& \cong \mathfrak{s p}(m),
\end{aligned}
$$

where $N=d_{r} m$. Let us consider the complexification of $\mathbb{R}^{m} \otimes \tilde{\Delta}_{r}$ and decompose it

$$
\left(\mathbb{R}^{m} \otimes \tilde{\Delta}_{r}\right) \otimes \mathbb{C}=\left\{v \otimes(\psi-i I \psi) \mid v \in \mathbb{R}^{m}, \psi \in \tilde{\Delta}_{r}\right\} \oplus\left\{v \otimes(\psi+i I \psi) \mid v \in \mathbb{R}^{m}, \psi \in \tilde{\Delta}_{r}\right\},
$$

where the first and second subspaces are the $+i$ and $-i$ eigenspaces of $\operatorname{Id}_{m} \otimes I$ respectively. Notice that

$$
\begin{aligned}
\left(\operatorname{Id}_{m} \otimes J\right)\left(v \otimes\left(\psi \mp i \operatorname{Id}_{m} \otimes I \psi\right)\right) & =v \otimes J \psi \mp i v \otimes J I \psi \\
& =v \otimes J \psi \pm i v \otimes I J \psi
\end{aligned}
$$

i.e. $\operatorname{Id}_{m} \otimes J$ interchanges the two subspaces and squares to $-\operatorname{Id}_{N}$. For any $\operatorname{Id}_{m} \otimes \xi \in \widehat{\operatorname{spin}(r)}$

$$
\begin{aligned}
\left(\operatorname{Id}_{m} \otimes \xi\right)(v \otimes(\psi \pm i I \psi)) & =v \otimes(\xi \psi \pm i \xi I \psi) \\
& =v \otimes(\xi \psi \pm i I \xi \psi)
\end{aligned}
$$

which means that the subspaces $\left\{v \otimes(\psi-i I \psi) \mid v \in \mathbb{R}^{m}, \psi \in \tilde{\Delta}_{r}\right\}$ and $\left\{v \otimes(\psi+i I \psi) \mid v \in \mathbb{R}^{m}, \psi \in \tilde{\Delta}_{r}\right\}$ are isomorphic to $\mathbb{C}^{m} \otimes \Delta_{r}$ as $\widehat{\mathfrak{s p i n}(r)}$ representations.

Now consider

$$
A \otimes \operatorname{Id}_{\tilde{\Delta}_{r}}+B \otimes I+C \otimes J+D \otimes K \in \mathfrak{s o}(m) \otimes \operatorname{Id}_{\tilde{\Delta}_{r}} \oplus S^{2} \mathbb{R}^{m} \otimes I \oplus S^{2} \mathbb{R}^{m} \otimes J \oplus S^{2} \mathbb{R}^{m} \otimes K=\mathfrak{s p}(m),
$$

and

$$
\begin{aligned}
\left(A \otimes \operatorname{Id}_{\tilde{\Delta}_{r}}+B \otimes I+C \otimes J+D \otimes K\right)(v \otimes(\psi+i I \psi))= & A v \otimes(\psi+i I \psi)+B v \otimes(I \psi+i I I \psi) \\
& +C v \otimes(J \psi+i J I \psi)+D v \otimes(K \psi+i K I \psi) \\
= & \left((A-i B) \otimes \operatorname{Id}_{\tilde{\Delta}_{r}}+(C+i D) \otimes J\right)(v \otimes(\psi+i I \psi)) .
\end{aligned}
$$

Similarly,

$\left(A \otimes \operatorname{Id}_{\tilde{\Delta}_{r}}+B \otimes I+C \otimes J+D \otimes K\right)(v \otimes(\psi-i I \psi))=\left((A+i B) \otimes \operatorname{Id}_{\tilde{\Delta}_{r}}+(C-i D) \otimes J\right)(v \otimes(\psi-i I \psi))$. If $C=D=0$, the subalgebra

$$
\widehat{\mathfrak{u}(m)_{I}}=\left\{A \otimes \operatorname{Id}_{\tilde{\Delta}_{r}}+B \otimes I \in \mathfrak{s o}(m) \otimes \operatorname{Id}_{\tilde{\Delta}_{r}} \oplus S^{2} \mathbb{R}^{m} \otimes I \mid A \in \mathfrak{s o}(m), B \in S^{2} \mathbb{R}^{m}\right\}
$$

is represented as follows

$$
\begin{aligned}
\tilde{\Delta}_{r} \otimes \mathbb{C} & =\mathbb{C}_{I}^{m} \otimes \Delta_{r} \oplus \overline{\mathbb{C}_{I}^{m}} \otimes \Delta_{r} \\
& =\left(\mathbb{C}_{I}^{m} \oplus \overline{\mathbb{C}_{I}^{m}}\right) \otimes \Delta_{r}
\end{aligned}
$$

where $\mathbb{C}_{I}^{m}$ and $\overline{\mathbb{C}_{I}^{m}}$ denote the standard representation of $\widehat{\mathfrak{u}(m)_{I}}$ and its conjugate respectively. Since $\operatorname{Id}_{m} \otimes J$ interchanges the two summands, squares to $-\operatorname{Id}_{N}$ and commutes with the action of $\widehat{\mathfrak{s p i n}(r)}$, we have the standard complex representation of $\mathfrak{s p}(m)$ as a factor

$$
\tilde{\Delta}_{r} \otimes \mathbb{C}=\mathbb{C}^{2 m} \otimes \Delta_{r}
$$

Thus, we have a representation

$$
S p(m) \times \operatorname{Spin}(r) \longrightarrow S O(N) \subset \operatorname{Aut}\left(\mathbb{C}^{2 m} \otimes \Delta_{r}\right) .
$$


Structure group. Since $\widehat{\mathfrak{s p}(m)}$ and $\widehat{\mathfrak{s p i n}(r)}$ commute with each other, we can take separately the exponentials of their elements within $\mathbb{C}(N)$. By considering (3), the exponential of an element $\Omega \otimes \operatorname{Id}_{\Delta_{r}} \in \mathfrak{s p}(m) \otimes \operatorname{Id}_{\Delta_{r}}=$ $\widehat{\mathfrak{s p i n}(r)}$ is

$$
e^{\Omega} \otimes \operatorname{Id}_{\tilde{\Delta}_{r}} \in \widehat{S p(m)}=S p(m) \otimes \operatorname{Id}_{\Delta_{r}} \cong S p(m) .
$$

On the other hand, if $\operatorname{Id}_{2 m} \otimes \xi \in \widehat{\mathfrak{s p i n}(r)}$, its exponential is

$$
\operatorname{Id}_{2 m} \otimes e^{\xi} \in \operatorname{Id}_{2 m} \otimes \kappa(\operatorname{Spin}(r))=\widehat{\operatorname{Spin}(r)} \cong \operatorname{Spin}(r),
$$

since $\operatorname{Spin}(r)$ is represented faithfully on $\Delta_{r}$. The image of $\operatorname{Sp}(m) \times \operatorname{Spin}(r)$ in $S O(N) \subset \operatorname{Aut}\left(\mathbb{C}^{2 m} \otimes \Delta_{r}\right)$ under the aforementioned representation is

$$
\mathcal{N}_{S O(N)}^{0}(\widehat{\operatorname{Spin}(r)})=\widehat{\operatorname{Sp}(m)} \widehat{\operatorname{Spin}(r)},
$$

the subgroup of all possible products of elements of the two subgroups, i.e. we have a map

$$
S p(m) \times \operatorname{Spin}(r) \stackrel{\rho}{\rightarrow} \widehat{\operatorname{Sp(m)} \widehat{\operatorname{Spin}(r)} \subset S O(N) .}
$$

Now we need to find $\operatorname{ker}(\rho)$ and identify $\widehat{\operatorname{Sp}(m)} \widehat{\operatorname{Spin}(r)}$ as a quotient

$$
\widehat{\operatorname{Sp}(m)} \widehat{\operatorname{Spin}(r)} \cong \frac{\operatorname{Sp}(m) \times \operatorname{Spin}(r)}{\operatorname{ker}(\rho)} .
$$

If there are elements $g \in \operatorname{Sp}(m)$ and $h \in \operatorname{Spin}(r)$ such that

$$
\rho(g, h)=\operatorname{Id}_{N},
$$

then

$$
\widehat{\operatorname{Spin}(r)} \ni \rho\left(\operatorname{Id}_{2 m}, h\right)=\rho(g, 1)^{-1} \in \widehat{\operatorname{Sp}(m)} .
$$

Since $\rho\left(\operatorname{Id}_{2 m}, h\right)$ commutes with every element of $\widehat{\operatorname{Sin}(r)}$, it belongs to its center $Z(\widehat{\operatorname{Spin}(r)}) \cong Z(\operatorname{Spin}(r))=$ $\mathbb{Z}_{2}=\{ \pm 1\}$. Note that -1 is mapped to $-\operatorname{Id}_{\Delta_{r}}$ under the $\operatorname{Spin}(r)$ representation, and that $\left(\operatorname{Id}_{2 m},-1\right)$ maps to $-\mathrm{Id}_{2 m} \otimes \operatorname{Id}_{\Delta_{r}} \in S O(N)$ under $\rho$. Note that $-\mathrm{Id}_{2 m} \otimes \operatorname{Id}_{\Delta_{r}}$ also belongs to $\widehat{S p(m)}$ being the image of $\left(-\mathrm{Id}_{2 m}, 1\right) \in S p(m) \times \operatorname{Spin}(r)$. Thus,

$$
\begin{aligned}
& \operatorname{ker}(\rho)=\left\{ \pm\left(\operatorname{Id}_{2 m}, 1\right)\right\} \cong \mathbb{Z}_{2}, \\
& \widehat{\operatorname{Sp}(m)} \widehat{\operatorname{Spin}(r)} \cong \frac{\operatorname{Sp}(m) \times \operatorname{Spin}(r)}{\mathbb{Z}_{2}} .
\end{aligned}
$$

Fundamental group. Clearly,

$$
\pi_{1}(\widehat{\operatorname{Sp}(m)} \widehat{\operatorname{Spin}(r)})=\mathbb{Z}_{2}
$$

\section{$3.5 r \equiv 4(\bmod 8)$}

Recall from [2] that

$$
\tilde{\Delta}_{r}^{ \pm}=\frac{1}{2}\left(1 \pm e_{1} \cdots e_{r}\right) \tilde{\Delta}_{r+3} .
$$

In this case, $\tilde{\Delta}_{r}^{ \pm}$admits three complex structure $I^{ \pm}, J^{ \pm}$and $K^{ \pm}$induced by Clifford multiplication with the elements $\frac{1}{2}\left(1 \pm e_{1} \ldots e_{r}\right) e_{r+1} e_{r+2}, \frac{1}{2}\left(1 \pm e_{1} \ldots e_{r}\right) e_{r+1} e_{r+3}$ and $\frac{1}{2}\left(1 \pm e_{1} \ldots e_{r}\right) e_{r+2} e_{r+3}$, respectively. Just as in the previous case,

$$
\tilde{\Delta}_{r}^{+} \otimes \mathbb{C}=\left\{\psi-i I^{+} \psi \mid \psi \in \tilde{\Delta}_{r}^{+}\right\} \oplus\left\{\psi+i I^{+} \psi \mid \psi \in \tilde{\Delta}_{r}^{+}\right\},
$$


and both summands are isomorphic to $\Delta_{r}^{-}$. Indeed, if

$$
\psi=\frac{1}{2}\left(1+e_{1} \cdots e_{r}\right) \cdot \phi \in \tilde{\Delta}_{r}^{+},
$$

then,

$$
(-i)^{r / 2}\left(e_{1} \cdots e_{r}\right) \cdot\left(\psi \pm i I^{+} \psi\right)=-\left(\psi \pm i I^{+} \psi\right),
$$

i.e. $\psi \pm i I^{+} \psi \in \Delta_{r}^{-}$. In other words,

$$
\tilde{\Delta}_{r}^{+} \otimes \mathbb{C}=\Delta_{r}^{-} .
$$

Similarly,

$$
\tilde{\Delta}_{r}^{-} \otimes \mathbb{C}=\Delta_{r}^{+} .
$$

The rest of the proof proceeds as in the previous case,

$$
\left(\mathbb{R}^{m_{1}} \otimes \tilde{\Delta}_{r}^{+} \oplus \mathbb{R}^{m_{2}} \tilde{\Delta}_{r}^{-}\right) \otimes \mathbb{C}=\mathbb{C}^{2 m_{1}} \otimes \Delta_{r}^{-} \oplus \mathbb{C}^{2 m_{2}} \Delta_{r}^{+},
$$

and we have a representation

$$
S p\left(m_{1}\right) \times S p\left(m_{2}\right) \times S p i n(r) \longrightarrow S O(N) \subset \operatorname{Aut}\left(\mathbb{C}^{N}\right),
$$

where $N=d_{r}\left(m_{1}+m_{2}\right)$.

Structure group. Since $\widehat{\mathfrak{s p}\left(m_{1}\right)} \oplus \widehat{\mathfrak{s p}\left(m_{2}\right)}$ and $\widehat{\mathfrak{s p i n}(r)}$ commute with each other, we can take separately the exponentials of their elements within $\mathbb{C}(N)$. The exponential of $\Omega_{1} \otimes \operatorname{Id}_{\Delta_{r}^{-}} \oplus \Omega_{2} \otimes \operatorname{Id}_{\Delta_{r}^{+}} \in \widehat{\mathfrak{s p}\left(m_{1}\right)} \oplus \widehat{\mathfrak{s p}\left(m_{2}\right)}$ gives

$$
\begin{aligned}
\left(\begin{array}{cc}
e^{\Omega_{1}} \otimes \operatorname{Id}_{\Delta_{r}^{-}} & \\
& e^{\Omega_{2}} \otimes \operatorname{Id}_{\Delta_{r}^{+}}
\end{array}\right) & \in\left(\begin{array}{cc}
\left.\widehat{S p\left(m_{1}\right.}\right) & \\
& \left.\widehat{S p\left(m_{2}\right.}\right)
\end{array}\right) \\
& =\left(\begin{array}{cc}
S p\left(m_{1}\right) \otimes \operatorname{Id}_{\Delta_{r}^{-}} & S p\left(m_{2}\right) \otimes \operatorname{Id}_{\Delta_{r}^{+}}
\end{array}\right) \\
& \cong S p\left(m_{1}\right) \times S p\left(m_{2}\right) .
\end{aligned}
$$

On the other hand, if $\operatorname{Id}_{2 m_{1}} \otimes \xi^{-} \oplus \operatorname{Id}_{2 m_{2}} \otimes \xi^{+} \in \widehat{\mathfrak{s p i n}(r)}$, its exponential is

$$
\begin{aligned}
\left(\begin{array}{cl}
\operatorname{Id}_{2 m_{1}} \otimes e^{\xi^{-}} & \operatorname{Id}_{2 m_{2}} \otimes e^{\xi^{+}}
\end{array}\right) & \in\left(\begin{array}{ll}
\operatorname{Id}_{2 m_{1}} \otimes \kappa^{-}(\operatorname{Spin}(r)) & \operatorname{Id}_{2 m_{2}} \otimes \kappa^{+}(\operatorname{Spin}(r))
\end{array}\right) \\
& = \begin{cases}\widehat{\operatorname{Spin}(r)} \cong \operatorname{Spin}(r) & \text { if } m_{1}>0 \text { and } m_{2}>0, \\
\widehat{\operatorname{Pin}(r)}-\cong \kappa^{-}(\operatorname{Spin}(r)) & \text { if } m_{1}>0 \text { and } m_{2}=0, \\
\widehat{\operatorname{Pin}(r)}+\cong \kappa^{+}(\operatorname{Spin}(r)) & \text { if } m_{1}=0 \text { and } m_{2}>0,\end{cases}
\end{aligned}
$$

where the first case is faithful and the last two are not, with

$$
\begin{aligned}
& \widehat{\operatorname{Spin}(r)^{ \pm}} \cong \kappa^{ \pm}(\operatorname{Spin}(r)) \cong \frac{\operatorname{Spin}(r)}{\left\{1, \mp \operatorname{vol}_{r}\right\}}, \quad \text { if } r>4 \\
& \widehat{\operatorname{Spin}(r)^{ \pm}} \cong \kappa^{ \pm}(\operatorname{Spin}(r)) \cong \operatorname{Spin}(3), \quad \text { if } r=4 .
\end{aligned}
$$

The images of $S p\left(m_{1}\right) \times S p\left(m_{2}\right) \times \operatorname{Spin}(r)$ in $S O(N) \subset \operatorname{Aut}\left(\mathbb{C}^{N}\right)$ under the aforementioned representations are

$$
\begin{aligned}
\mathcal{N}_{S O(N)}^{0}(\widehat{\operatorname{Spin}(r)}) & \left.=\left(\widehat{\operatorname{Sp(m_{1}}}\right) \times \widehat{\left.\operatorname{Sp(m_{2}}\right)}\right) \widehat{\operatorname{Spin}(r)}, \\
\mathcal{N}_{S O(N)}^{0}(\widehat{\operatorname{Sin}(r)}-) & \left.=\widehat{\operatorname{Sp(m_{1}}}\right) \widehat{\operatorname{Spin}(r)}- \\
\mathcal{N}_{S O(N)}^{0}(\widehat{\operatorname{Spin}(r)}+) & \left.=\widehat{\operatorname{Sp(m_{2}}}\right) \widehat{\operatorname{Spin}(r)}+
\end{aligned}
$$


respectively, i.e. we have maps

$$
\begin{aligned}
& \left.\left.S p\left(m_{1}\right) \times S p\left(m_{2}\right) \times \operatorname{Spin}(r) \stackrel{\rho}{\rightarrow}\left(\widehat{\operatorname{Sp(m_{1}}}\right) \times \widehat{\operatorname{Sp(m_{2}}}\right)\right) \widehat{\operatorname{Spin}(r)} \subset S O(N), \\
& \operatorname{Sp}\left(m_{1}\right) \times \operatorname{Spin}(r) \stackrel{\rho}{\rightarrow} \quad \widehat{S p\left(m_{1}\right)} \widehat{S p i n(r)}-\subset S O(N), \\
& \operatorname{Sp}\left(m_{2}\right) \times \operatorname{Spin}(r) \stackrel{\rho}{\rightarrow} \widehat{S p\left(m_{2}\right)} \widehat{\operatorname{Spin}(r)}+\subset S O(N) .
\end{aligned}
$$

Now we need to find $\operatorname{ker}(\rho)$ en each case to identify the relevant group as a quotient.

- Case $m_{1}, m_{2}>0$. If there are elements $g_{i} \in S p\left(m_{i}\right)$ and $h \in \operatorname{Spin}(r)$ such that

$$
\rho\left(g_{1}, g_{2}, h\right)=\operatorname{Id}_{N}
$$

then

$$
\widehat{\operatorname{Spin}(r)} \ni \rho\left(\operatorname{Id}_{2 m_{1}}, \operatorname{Id}_{2 m_{2}}, h\right)=\rho\left(g_{1}, g_{2}, 1\right)^{-1} \in \widehat{\left.\operatorname{Sp(m_{1}}\right)} \times \widehat{S p\left(m_{2}\right)} .
$$

Since $\rho\left(\operatorname{Id}_{2 m_{1}}, \operatorname{Id}_{2 m_{2}}, h\right)$ commutes with every element of $\left.\widehat{\operatorname{Spin}(r}\right)$, it belongs to its center $\left.Z(\widehat{\operatorname{Spin}(r})\right) \cong$ $Z(\operatorname{Spin}(r))=\left\{1,-1, \operatorname{vol}_{r},-\operatorname{vol}_{r}\right\} \cong \mathbb{Z}_{2} \oplus \mathbb{Z}_{2}$. The element -1 is mapped to $-\operatorname{Id}_{\Delta_{r}^{ \pm}}$in the $\operatorname{Spin}(r)$ representations $\Delta_{r}^{ \pm}$, and $\left(\operatorname{Id}_{2 m_{1}}, \operatorname{Id}_{2 m_{2}},-1\right)$ is mapped to $-\left(\operatorname{Id}_{2 m_{1}} \otimes \operatorname{Id}_{\Delta_{r}^{-}} \oplus \operatorname{Id}_{2 m_{2}} \otimes \operatorname{Id}_{\Delta_{r}^{+}}\right) \in S O(N)$. The element $\operatorname{vol}_{r}$ is mapped to $\mp \mathrm{Id}_{\Delta_{r}^{ \pm}}$in the $\operatorname{Spin}(r)$ representations $\Delta_{r}^{ \pm}$, and $\left(\operatorname{Id}_{2 m_{1}}, \mathrm{Id}_{2 m_{2}}, \mathrm{vol}_{r}\right)$ is mapped to $\left(\operatorname{Id}_{2 m_{1}} \otimes \operatorname{Id}_{\Delta_{r}^{-}} \oplus(-1) \operatorname{Id}_{2 m_{2}} \otimes \operatorname{Id}_{\Delta_{r}^{+}}\right) \in S O\left(d_{r}\left(m_{1}+m_{2}\right)\right)$. In this case, $-\left(\operatorname{Id}_{2 m_{1}} \otimes \operatorname{Id}_{\Delta_{r}^{-}} \oplus \operatorname{Id}_{2 m_{2}} \otimes \operatorname{Id}_{\Delta_{r}^{+}}\right)$ and $\left(\operatorname{Id}_{2 m_{1}} \otimes \operatorname{Id}_{\Delta_{r}^{-}} \oplus(-1) \operatorname{Id}_{2 m_{2}} \otimes \operatorname{Id}_{\Delta_{r}^{+}}\right)$belong to $\left.\left.\widehat{S p\left(m_{1}\right.}\right) \times \widehat{S p\left(m_{2}\right.}\right)$. Thus,

$$
\begin{aligned}
& \operatorname{ker}(\rho)=\left\{\left(\operatorname{Id}_{2 m_{1}}, \operatorname{Id}_{2 m_{2}}, 1\right),\left(-\mathrm{Id}_{2 m_{1}},-\mathrm{Id}_{2 m_{2}},-1\right),\right. \\
& \left.\left(\mathrm{Id}_{2 m_{1}},-\mathrm{Id}_{2 m_{2}}, \operatorname{vol}_{r}\right),\left(-\mathrm{Id}_{2 m_{1}}, \mathrm{Id}_{2 m_{2}},-\mathrm{vol}_{r}\right)\right\} \text {, } \\
& \left(\widehat{\operatorname{Sp}\left(m_{1}\right)} \times \widehat{\operatorname{Sp}\left(m_{2}\right)}\right) \widehat{\operatorname{Spin}(r)} \cong \frac{\operatorname{Sp}\left(m_{1}\right) \times S p\left(m_{2}\right) \times \operatorname{Spin}(r)}{\mathbb{Z}_{2} \oplus \mathbb{Z}_{2}} .
\end{aligned}
$$

- Case $m_{1}>0, m_{2}=0$. If there are elements $g_{1} \in S p\left(m_{1}\right)$ and $h \in \operatorname{Spin}(r)$ such that

$$
\rho\left(g_{1}, h\right)=\operatorname{Id}_{N},
$$

then

$$
\rho\left(\operatorname{Id}_{2 m_{1}}, h\right)=\rho\left(g_{1}, 1\right)^{-1} \in \widehat{\left.\operatorname{Sp(m_{1}}\right)}
$$

and

$$
\rho\left(\operatorname{Id}_{2 m_{1}}, h\right) \in \widehat{S p i n(r)}-\cap \widehat{S p\left(m_{1}\right)} .
$$

Since $\rho\left(\operatorname{Id}_{2 m_{1}}, h\right)$ commutes with every element of $\left.\widehat{\operatorname{Spin}(r)}\right)^{-}$, it belongs to its center

$$
\left.Z(\widehat{\operatorname{Sin}(r)})^{-}\right) \cong \begin{cases}Z\left(\kappa^{-}(\operatorname{Spin}(r))\right)=Z\left(\operatorname{Spin}(r) /\left\{1, \operatorname{vol}_{r}\right\}\right)=\left\{1,-1, \operatorname{vol}_{r},-\operatorname{vol}_{r}\right\} /\left\{1, \operatorname{vol}_{r}\right\} \cong\{1,-1\} \cong \mathbb{Z}_{2} & \text { if } r>4 . \\ Z\left(\kappa^{-}(\operatorname{Spin}(r))\right)=Z(\{1\} \times \operatorname{Spin}(3))=\{(1,1),(1,-1)\} \cong \mathbb{Z}_{2} & \text { if } r=4 .\end{cases}
$$

- If $r>4$, the element -1 is mapped to $-\operatorname{Id}_{\Delta_{r}^{-}}$in the $\operatorname{Spin}(r)$ representation $\Delta_{r}^{-}$, and $\left(\operatorname{Id}_{2 m_{1}},-1\right)$ is mapped to $-\left(\operatorname{Id}_{2 m_{1}} \otimes \operatorname{Id}_{\Delta_{r}^{-}}\right) \in S O(N)$. In this case, $-\operatorname{Id}_{2 m_{1}} \otimes \operatorname{Id}_{\Delta_{r}^{-}}$belongs to $\left.\widehat{S p\left(m_{1}\right.}\right)$. Thus,

$$
\begin{aligned}
\operatorname{ker}(\rho) & =\left\{\left(\operatorname{Id}_{2 m_{1}}, 1\right),\left(\operatorname{Id}_{2 m_{1}}, \operatorname{vol}_{r}\right),\left(-\operatorname{Id}_{2 m_{1}},-1\right),\left(-\operatorname{Id}_{2 m_{1}},-\operatorname{vol}_{r}\right)\right\}, \\
\widehat{\operatorname{Sp}\left(m_{1}\right)} \widehat{\operatorname{Spin}(r)}- & \cong \frac{\operatorname{Sp}\left(m_{1}\right) \times \operatorname{Spin}(r)}{\mathbb{Z}_{2} \oplus \mathbb{Z}_{2}} .
\end{aligned}
$$

- If $r=4$, the element $(1,-1) \in\{1\} \times \operatorname{Spin}(3)$ is mapped to $-\operatorname{Id}_{\Delta_{r}^{-}}$in the $\operatorname{Spin}(r)$ representation $\Delta_{r}^{-}$, and $\left(\operatorname{Id}_{2 m_{1}},(1,-1)\right)$ is mapped to $-\left(\operatorname{Id}_{2 m_{1}} \otimes \operatorname{Id}_{\Delta_{r}^{-}}\right) \in S O(N)$. In this case, $-\operatorname{Id}_{2 m_{1}} \otimes \operatorname{Id}_{\Delta_{r}^{-}}$ belongs to $\widehat{S p\left(m_{1}\right)}$. Thus,

$$
\begin{aligned}
\operatorname{ker}(\rho) & =\left\{\left(\operatorname{Id}_{2 m_{1}},(1,1)\right),\left(-\operatorname{Id}_{2 m_{1}},(1,-1)\right)\right\} \times(\operatorname{Spin}(3) \times\{1\}), \\
\widehat{\left.\operatorname{Sp(m_{1}}\right)} \widehat{\operatorname{Spin}(4)}- & \cong \frac{\operatorname{Sp}\left(m_{1}\right) \times(\operatorname{Spin}(3) \times \operatorname{Spin}(3))}{\left\{\left(\operatorname{Id}_{2 m_{1}},(1,1)\right),\left(-\mathrm{Id}_{2 m_{1}},(1,-1)\right)\right\} \times(\operatorname{Spin}(3) \times\{1\})} \cong \frac{\operatorname{Sp}\left(m_{1}\right) \times \operatorname{Spin}(3)}{\mathbb{Z}_{2}} .
\end{aligned}
$$

Note that $(1,-1) \in \operatorname{Spin}(3) \times \operatorname{Spin}(3)$ corresponds $-\operatorname{vol}_{4} \in \operatorname{Spin}(4)$. 
- Case $m_{1}=0, m_{2}>0$. If there are elements $g_{2} \in S p\left(m_{2}\right)$ and $h \in \operatorname{Spin}(r)$ such that

$$
\rho\left(g_{2}, h\right)=\operatorname{Id}_{N}
$$

then

$$
\rho\left(\operatorname{Id}_{2 m_{2}}, h\right)=\rho\left(g_{2}, 1\right)^{-1} \in \widehat{\operatorname{Sp(m_{2})}}
$$

and

$$
\rho\left(\operatorname{Id}_{2 m_{2}}, h\right) \in \widehat{\operatorname{Sin}(r)}+\cap \widehat{\operatorname{Sp(m_{2})}} .
$$

Since $\rho\left(\operatorname{Id}_{2 m_{2}}, h\right)$ commutes with every element of $\widehat{\operatorname{Sin}(r)}+$, it belongs to its center

$$
Z(\widehat{\operatorname{Spin}(r)}-) \cong \begin{cases}Z\left(\kappa^{+}(\operatorname{Spin}(r))\right)=Z\left(\operatorname{Spin}(r) /\left\{1,-\operatorname{vol}_{r}\right\}\right)=\left\{1,-1, \operatorname{vol}_{r},-\operatorname{vol}_{r}\right\} /\left\{1,-\operatorname{vol}_{r}\right\} \cong\{1,-1\} \cong \mathbb{Z}_{2} & \text { if } r>4 . \\ Z\left(\kappa^{+}(\operatorname{Spin}(r))\right)=Z(\operatorname{Spin}(3) \times\{1\})=\{(1,1),(-1,1)\} \cong \mathbb{Z}_{2} & \text { if } r=4 .\end{cases}
$$

- The element -1 is mapped to $-\operatorname{Id}_{\Delta_{r}^{+}}$in the $\operatorname{Spin}(r)$ representations $\Delta_{r}^{+}$, and $\left(\operatorname{Id}_{2 m_{1}},-1\right)$ is mapped to $-\left(\operatorname{Id}_{2 m_{2}} \otimes \operatorname{Id}_{\Delta_{r}^{+}}\right) \in S O(N)$. In this case, $-\operatorname{Id}_{2 m_{2}} \otimes \operatorname{Id}_{\Delta_{r}^{-}}$belongs to $\left.\widehat{\operatorname{Sp}\left(m_{2}\right.}\right)$. Thus,

$$
\begin{aligned}
\operatorname{ker}(\rho) & =\left\{\left(\operatorname{Id}_{2 m_{2}}, 1\right),\left(\mathrm{Id}_{2 m_{2}},-\operatorname{vol}_{r}\right),\left(-\mathrm{Id}_{2 m_{2}},-1\right),\left(-\mathrm{Id}_{2 m_{2}}, \operatorname{vol}_{r}\right)\right\} \\
\widehat{\operatorname{Sp}\left(m_{2}\right)} \widehat{\operatorname{Spin}(r)}+ & \cong \frac{\operatorname{Sp}\left(m_{2}\right) \times \operatorname{Spin}(r)}{\mathbb{Z}_{2} \oplus \mathbb{Z}_{2}} .
\end{aligned}
$$

- If $r=4$, the element $(-1,1) \in \operatorname{Spin}(3) \times\{1\}$ is mapped to $-\operatorname{Id}_{\Delta_{r}^{+}}$in the $\operatorname{Spin}(r)$ representation $\Delta_{r}^{+}$, and $\left(\operatorname{Id}_{2 m_{2}},(-1,1)\right)$ is mapped to $-\left(\operatorname{Id}_{2 m_{2}} \otimes \operatorname{Id}_{\Delta_{r}^{+}}\right) \in S O(N)$. In this case, $-\operatorname{Id}_{2 m_{2}} \otimes \mathrm{Id}_{\Delta_{r}^{-}}$ belongs to $\widehat{S p\left(m_{2}\right)}$. Thus,

$$
\begin{aligned}
\operatorname{ker}(\rho) & =\left\{\left(\operatorname{Id}_{2 m_{2}},(1,1)\right),\left(-\mathrm{Id}_{2 m_{2}},(-1,1)\right)\right\} \times(\operatorname{Spin}(3) \times\{1\}), \\
\widehat{\operatorname{Sp}\left(m_{2}\right)} \widehat{\operatorname{Spin}(4)}+ & \cong \frac{\operatorname{Sp}\left(m_{2}\right) \times(\operatorname{Spin}(3) \times \operatorname{Spin}(3))}{\left\{\left(\operatorname{Id}_{2 m_{2}},(1,1)\right),\left(-\mathrm{Id}_{2 m_{2}},(-1,1)\right)\right\} \times(\{1\} \times \operatorname{Spin}(3))} \cong \frac{\operatorname{Sp}\left(m_{2}\right) \times \operatorname{Spin}(3)}{\mathbb{Z}_{2}} .
\end{aligned}
$$

Note that $(-1,1) \in \operatorname{Spin}(3) \times \operatorname{Spin}(3)$ corresponds $\operatorname{vol}_{4} \in \operatorname{Spin}(4)$.

Fundamental group. Clearly,

$$
\begin{aligned}
& \left.\pi_{1}\left(\left(\widehat{\operatorname{Sp}\left(m_{1}\right)}\right) \times \widehat{\operatorname{Sp}\left(m_{2}\right)}\right) \widehat{\operatorname{Spin}(r)}\right)=\mathbb{Z}_{2} \oplus \mathbb{Z}_{2}, \\
& \pi_{1}\left(\widehat{\left.\operatorname{Sp(m_{1}}\right)} \widehat{\operatorname{Spin}(r)}-\right) \cong \begin{cases}\mathbb{Z}_{2} \oplus \mathbb{Z}_{2}, & \text { if } m_{1}>0, m_{2}=0, r>4, \\
\mathbb{Z}_{2}, & \text { if } m_{1}>0, m_{2}=0, r=4,\end{cases} \\
& \pi_{1}\left(\widehat{\operatorname{Sp(m_{2})}} \widehat{\operatorname{Spin}(r)}+\right) \cong \begin{cases}\mathbb{Z}_{2} \oplus \mathbb{Z}_{2}, & \text { if } m_{1}=0, m_{2}>0, r>4, \\
\mathbb{Z}_{2} & \text { if } m_{1}=0, m_{2}>0, r=4 .\end{cases}
\end{aligned}
$$

Thus, we have proved the following three theorems.

Theorem 3.1 The complexification of a real representation $\mathbb{R}^{N}$ of $C l_{r}^{0}$ without trivial summands decomposes as follows

\begin{tabular}{|c|c|}
\hline$r(\bmod 8)$ & $\mathbb{R}^{N} \otimes \mathbb{C}$ \\
\hline 0 & $\mathbb{C}^{m_{1}} \otimes \Delta_{r}^{+} \oplus \mathbb{C}^{m_{2}} \otimes \Delta_{r}^{-}$ \\
\hline 1,7 & $\mathbb{C}^{m} \otimes \Delta_{r}$ \\
\hline 2 & $\mathbb{C}^{m} \otimes \Delta_{r}^{+} \oplus \overline{\mathbb{C}^{m}} \otimes \Delta_{r}^{-}$ \\
\hline 6 & $\overline{\mathbb{C}^{m}} \otimes \Delta_{r}^{+} \oplus \mathbb{C}^{m} \otimes \Delta_{r}^{-}$ \\
\hline 3,5 & $\mathbb{C}^{2 m} \otimes \Delta_{r}$ \\
\hline 4 & $\mathbb{C}^{2 m_{2}} \otimes \Delta_{r}^{+} \oplus \mathbb{C}^{2 m_{1}} \otimes \Delta_{r}^{-}$ \\
\hline
\end{tabular}


where the different $\mathbb{C}^{s}$ denote the corresponding standard complex representations of the classical Lie algebras $\mathfrak{s o}(s), \mathfrak{u}(s)$ or $\mathfrak{s p}(s / 2)$.

Theorem 3.2 The connected components of the identity $\mathcal{N}_{S O(N)}^{0}(\widehat{\operatorname{Spin}(r)})$ of the normalizers $\mathcal{N}_{S O(N)}(\widehat{\operatorname{Spin}(r)})$ are isomorphic to the following groups:

- If $r \equiv 1,7(\bmod 8), N=d_{r} m$ and

$$
\mathcal{N}_{S O(N)}^{0}(\widehat{\operatorname{Spin}(r)}) \cong \begin{cases}\frac{S O(m) \times \operatorname{Spin}(r)}{\mathbb{Z}_{2}}, & \text { if } m \text { is even, } \\ S O(m) \times \operatorname{Spin}(r), & \text { if } m \text { is odd }\end{cases}
$$

- If $r \equiv 0(\bmod 8), N=d_{r}\left(m_{1}+m_{2}\right)$ and

$$
\begin{aligned}
\mathcal{N}_{S O(N)}^{0}(\widehat{\operatorname{Spin}(r)}) \cong \begin{cases}S O\left(m_{1}\right) \times S O\left(m_{2}\right) \times \operatorname{Spin}(r), & \text { if } m_{1}>0, m_{2}>0, m_{1} \equiv m_{2} \equiv 1(\bmod 2), \\
\frac{S O\left(m_{1}\right) \times S O\left(m_{2}\right) \times \operatorname{Spin}(r)}{\mathbb{Z}_{2}}, & \text { if } m_{1}>0, m_{2}>0, m_{1}+m_{2} \equiv 1(\bmod 2), \\
\frac{S O\left(m_{1}\right) \times S O\left(m_{2}\right) \times \operatorname{Spin}(r)}{\mathbb{Z}_{2} \oplus \mathbb{Z}_{2}}, & \text { if } m_{1}>0, m_{2}>0, m_{1} \equiv m_{2} \equiv 0(\bmod 2),\end{cases} \\
\left.\mathcal{N}_{S O(N)}^{0}(\widehat{\operatorname{Spin}(r)})^{+}\right) \cong \begin{cases}\frac{S O\left(m_{1}\right) \times \operatorname{Spin}(r)}{\mathbb{Z}_{2}}, & \text { if } m_{1}>0, m_{2}=0, m_{1} \equiv 1(\bmod 2), \\
\frac{S O\left(m_{1}\right) \times \operatorname{Spin}(r)}{\mathbb{Z}_{2} \oplus \mathbb{Z}_{2}}, & \text { if } m_{1}>0, m_{2}=0, m_{1} \equiv 0(\bmod 2),\end{cases} \\
\mathcal{N}_{S O(N)}^{0}(\widehat{\operatorname{Spin}(r)}-) \cong \begin{cases}\frac{S O\left(m_{2}\right) \times \operatorname{Spin}(r)}{\mathbb{Z}_{2}}, & \text { if } m_{1}=0, m_{2}>0, m_{2} \equiv 1(\bmod 2), \\
\frac{S O\left(m_{2}\right) \times \operatorname{Spin}(r)}{\mathbb{Z}_{2} \oplus \mathbb{Z}_{2}}, & \text { if } m_{1}=0, m_{2}>0, m_{2} \equiv 0(\bmod 2) .\end{cases}
\end{aligned}
$$

- If $r \equiv 2,6(\bmod 8), N=d_{r} m$ and

$$
\mathcal{N}_{S O(N)}^{0}(\widehat{\operatorname{Spin}(r)}) \cong \frac{U(m) \times \operatorname{Spin}(r)}{\mathbb{Z}_{4}} .
$$

- If $r \equiv 3,5(\bmod 8), N=d_{r} m$ and

$$
\mathcal{N}_{S O(N)}^{0}(\widehat{\operatorname{Spin}(r)}) \cong \frac{\operatorname{Sp}(m) \times \operatorname{Spin}(r)}{\mathbb{Z}_{2}}
$$

- If $r \equiv 4(\bmod 8), N=d_{r}\left(m_{1}+m_{2}\right)$ and

$$
\begin{gathered}
\mathcal{N}_{S O(N)}^{0}(\widehat{\operatorname{Spin}(r)}) \cong \frac{S p\left(m_{1}\right) \times S p\left(m_{2}\right) \times \operatorname{Spin}(r)}{\mathbb{Z}_{2} \oplus \mathbb{Z}_{2}}, \quad \text { if } m_{1}>0, m_{2}>0, \\
\mathcal{N}_{S O(N)}^{0}(\widehat{\operatorname{Spin}(r)}-) \cong \begin{cases}\frac{S p\left(m_{1}\right) \times \operatorname{Spin}(r)}{\mathbb{Z}_{2} \oplus \mathbb{Z}_{2}} & \text { if } m_{1}>0, m_{2}=0, r>4, \\
\frac{S p\left(m_{1}\right) \times \operatorname{Spin}(3)}{\mathbb{Z}_{2}} & \text { if } m_{1}>0, m_{2}=0, r=4,\end{cases} \\
\mathcal{N}_{S O(N)}^{0}(\widehat{\operatorname{Spin}(r)}+) \cong \begin{cases}\frac{S p\left(m_{2}\right) \times \operatorname{Spin}(r)}{\mathbb{Z}_{2} \oplus \mathbb{Z}_{2}} & \text { if } m_{1}=0, m_{2}>0, r>4, \\
\frac{S p\left(m_{2}\right) \times \operatorname{Spin}(3)}{\mathbb{Z}_{2}} & \text { if } m_{1}=0, m_{2}>0, r=4 .\end{cases}
\end{gathered}
$$

Theorem 3.3 The fundamental group of the connected components of the identity of the normalizers $\mathcal{N}_{S O(N)}^{0}(\widehat{\operatorname{Spin}(r)})$ are the following. 
- If $r \equiv 1,7(\bmod 8), N=d_{r} m$ and

$$
\pi_{1}\left(\mathcal{N}_{S O(N)}^{0}(\widehat{\operatorname{Spin}(r)})\right) \cong \begin{cases}\mathbb{Z}_{2} \oplus \mathbb{Z}_{2}, & \text { if } m \geq 4, m \equiv 0(\bmod 4), \\ \mathbb{Z}_{4}, & \text { if } m \geq 4, m \equiv 2(\bmod 4), \\ \mathbb{Z}_{2}, & \text { if } m>1 \text { and odd, } \\ \{1\}, & \text { if } m=1, \\ \mathbb{Z}, & \text { if } m=2 .\end{cases}
$$

- If $r \equiv 0(\bmod 8), N=d_{r}\left(m_{1}+m_{2}\right)$ and either $\left.\pi_{1}\left(\mathcal{N}_{S O(N)}^{0}(\widehat{\operatorname{Spin}(r})\right)\right)$ or $\pi_{1}\left(\mathcal{N}_{S O(N)}^{0}\left(\widehat{\operatorname{Spin}(r)^{+}}\right)\right)$or $\pi_{1}\left(\mathcal{N}_{S O(N)}^{0}(\widehat{\operatorname{Spin}(r)}-)\right)$ are isomorphic to

\begin{tabular}{|c|c|c|c|c|c|c|}
\hline$m_{1} m_{2}$ & 0 & 1 & 2 & $1(\bmod 2)$ & $2(\bmod 4)$ & $0(\bmod 4)$ \\
\hline 0 & & $\mathbb{Z}_{2}$ & $\mathbb{Z} \oplus \mathbb{Z}_{2}$ & $\mathbb{Z}_{2} \oplus \mathbb{Z}_{2}$ & $\mathbb{Z}_{2} \oplus \mathbb{Z}_{4}$ & $\mathbb{Z}_{2} \oplus \mathbb{Z}_{2} \oplus \mathbb{Z}_{2}$ \\
\hline 1 & $\mathbb{Z}_{2}$ & $\{1\}$ & $\mathbb{Z}$ & $\mathbb{Z}_{2}$ & $\mathbb{Z}_{4}$ & $\mathbb{Z}_{2} \oplus \mathbb{Z}_{2}$ \\
\hline 2 & $\mathbb{Z} \oplus \mathbb{Z}_{2}$ & $\mathbb{Z}$ & $\mathbb{Z} \oplus \mathbb{Z}$ & $\mathbb{Z} \oplus \mathbb{Z}_{2}$ & $\mathbb{Z} \oplus \mathbb{Z}_{4}$ & $\mathbb{Z} \oplus \mathbb{Z}_{2} \oplus \mathbb{Z}_{2}$ \\
\hline $1(\bmod 2)$ & $\mathbb{Z}_{2} \oplus \mathbb{Z}_{2}$ & $\mathbb{Z}_{2}$ & $\mathbb{Z} \oplus \mathbb{Z}_{2}$ & $\mathbb{Z}_{2} \oplus \mathbb{Z}_{2}$ & $\mathbb{Z}_{2} \oplus \mathbb{Z}_{4}$ & $\mathbb{Z}_{2} \oplus \mathbb{Z}_{2} \oplus \mathbb{Z}_{2}$ \\
\hline $2(\bmod 4)$ & $\mathbb{Z}_{2} \oplus \mathbb{Z}_{4}$ & $\mathbb{Z}_{4}$ & $\mathbb{Z} \oplus \mathbb{Z}_{4}$ & $\mathbb{Z}_{2} \oplus \mathbb{Z}_{4}$ & $\mathbb{Z}_{4} \oplus \mathbb{Z}_{4}$ & $\mathbb{Z}_{2} \oplus \mathbb{Z}_{2} \oplus \mathbb{Z}_{4}$ \\
\hline $0(\bmod 4)$ & $\mathbb{Z}_{2} \oplus \mathbb{Z}_{2} \oplus \mathbb{Z}_{2}$ & $\mathbb{Z}_{2} \oplus \mathbb{Z}_{2}$ & $\mathbb{Z} \oplus \mathbb{Z}_{2} \oplus \mathbb{Z}_{2}$ & $\mathbb{Z}_{2} \oplus \mathbb{Z}_{2} \oplus \mathbb{Z}_{2}$ & $\mathbb{Z}_{2} \oplus \mathbb{Z}_{2} \oplus \mathbb{Z}_{4}$ & $\mathbb{Z}_{2} \oplus \mathbb{Z}_{2} \oplus \mathbb{Z}_{2} \oplus \mathbb{Z}_{2}$ \\
\hline
\end{tabular}

depending on whether $m_{1}, m_{2}>0$ or $m_{1}=0$ or $m_{2}=0$ respectively.

- If $r \equiv 2,6(\bmod 8), N=d_{r} m$ and

$$
\left.\pi_{1}\left(\mathcal{N}_{S O(n)}^{0}(\widehat{\operatorname{Spin}(r})\right)\right)= \begin{cases}\mathbb{Z}, & \text { if }(m, 4)=1, \\ \mathbb{Z} \times \mathbb{Z}_{2}, & \text { if }(m, 4)=2, \\ \mathbb{Z} \times \mathbb{Z}_{4}, & \text { if }(m, 4)=4 .\end{cases}
$$

- If $r \equiv 3,5(\bmod 8), N=d_{r} m$ and

$$
\left.\pi_{1}\left(\mathcal{N}_{S O(N)}^{0}(\widehat{\operatorname{Spin}(r})\right)\right)=\mathbb{Z}_{2}
$$

- If $r \equiv 4(\bmod 8), N=d_{r}\left(m_{1}+m_{2}\right)$ and

$$
\begin{aligned}
\pi_{1}\left(\mathcal{N}_{S O(N)}^{0}(\widehat{\operatorname{Sin}(r)})\right) & \cong \mathbb{Z}_{2} \oplus \mathbb{Z}_{2}, \quad \text { if } m_{1}>0, m_{2}>0, \\
\pi_{1}\left(\mathcal{N}_{S O(N)}^{0}(\widehat{\operatorname{Sin}(r)}-)\right) & \cong \begin{cases}\mathbb{Z}_{2} \oplus \mathbb{Z}_{2}, & \text { if } m_{1}>0, m_{2}=0, r>4, \\
\mathbb{Z}_{2}, & \text { if } m_{1}>0, m_{2}=0, r=4,\end{cases} \\
\pi_{1}\left(\mathcal{N}_{S O(N)}^{0}(\widehat{\operatorname{Sin}(r)}+)\right) & \cong \begin{cases}\mathbb{Z}_{2} \oplus \mathbb{Z}_{2}, & \text { if } m_{1}=0, m_{2}>0, r>4, \\
\mathbb{Z}_{2} & \text { if } m_{1}=0, m_{2}>0, r=4 .\end{cases}
\end{aligned}
$$

\section{Lifting maps to the Spin group}

In this section, we will check how the generators of the fundamental groups $\pi_{1}\left(\mathcal{N}_{S O(N)}^{0}(S)\right)$ map into $\pi_{1}(S O(N))$.

Theorem 4.1 Let $r \geq 3$. There exist lifts

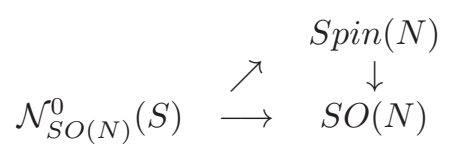

where $S$ denotes the homomorphic image of $\operatorname{Spin}(r)$ in $S O(N)$ (either $\widehat{\operatorname{Spin}(r)}$ or $\widehat{\operatorname{Spin}(r)})^{ \pm}$), in the following cases: 
- $\underline{r \equiv 1,7(\bmod 8)}$.

- For all $m \in \mathbb{N}$.

- $r \equiv 0(\bmod 8)$

- For all $m_{1}, m_{2} \in \mathbb{N}$ if $r>8$.

- For $m_{1} \equiv m_{2} \equiv 0(\bmod 2)$ if $r=8$.

- $\underline{r \equiv 2,6(\bmod 8)}$

- For all $m \in \mathbb{N}$ if $r>6$.

- For $m$ even if $r=6$.

- $\underline{r \equiv 3,5(\bmod 8)}$

- For all $m \in \mathbb{N}$ if $r>3$.

- For $m$ even if $r=3$.

- $r \equiv 4(\bmod 8)$

- For all $m_{1}, m_{2} \in \mathbb{N}$ if $r>4$.

- For $m_{1} \equiv m_{2} \equiv 0(\bmod 2)$ if $r=4$.

The rest of this section is devoted to prove Theorem 4.1 in a case by case analysis.

\section{1 $r \equiv 1,7(\bmod 8)$}

Recall

$$
\pi_{1}\left(\widehat{\operatorname{SO(m}) \widehat{\operatorname{Spin}(r)})}= \begin{cases}\langle(-1,1)\rangle \times\left\langle\left(\operatorname{vol}_{m},-1\right)\right\rangle \subset \operatorname{Spin}(m) \times \operatorname{Spin}(r), & \text { if } m \geq 4, m \equiv 0(\bmod 4), \\ \left\langle\left(\operatorname{vol}_{m},-1\right)\right\rangle \subset \operatorname{Spin}(m) \times \operatorname{Spin}(r), & \text { if } m \geq 4, m \equiv 2(\bmod 4), \\ \langle(-1,1)\rangle \subset \operatorname{Spin}(m) \times \operatorname{Spin}(r), & \text { if } m \geq 3, m \text { is odd, } \\ \{1\} \subset\{1\} \times \operatorname{Spin}(r), & \text { if } m=1, \\ \langle(\pi,-1)\rangle \subset \mathbb{R} \times \operatorname{Spin}(r), & \text { if } m=2 .\end{cases}\right.
$$

Thus, we only need to check the loops in $S O\left(d_{r} m\right)$ which are images of paths joining $(1,1)$ to either $(-1,1)$ or $\left(\operatorname{vol}_{m},-1\right)$ in $\operatorname{Spin}(m) \times \operatorname{Spin}(r)$ or joining $(0,1)$ to $(\pi,-1)$ in $\mathbb{R} \times \operatorname{Spin}(r)$.

- Consider the path

$$
\begin{aligned}
\delta_{1}:[0,1] & \longrightarrow \operatorname{Spin}(m) \times \operatorname{Spin}(r) \\
t & \mapsto\left(\cos (\pi t)+\sin (\pi t) v_{1} v_{2}, 1\right)
\end{aligned}
$$

joining $(1,1)$ to $(-1,1)$ in $\operatorname{Spin}(m) \times \operatorname{Spin}(r)$. It projects to the loop

$$
\begin{aligned}
\hat{\delta}_{1}:[0,1] & \longrightarrow \widehat{\operatorname{SO(m)} \widehat{\operatorname{Spin}(r)} \subset S O(N)} \\
t & \mapsto\left(\begin{array}{ccccc}
\cos (2 \pi t) & -\sin (2 \pi t) & & \\
\sin (2 \pi t) & \cos (2 \pi t) & & & \\
& & 1 & & \\
& & \ddots & \\
& & & 1
\end{array}\right)_{m \times m} \otimes \operatorname{Id}_{\Delta_{r}},
\end{aligned}
$$

which contains $2^{\left[\frac{r}{2}\right]}$ blocks

$$
\left(\begin{array}{cc}
\cos (2 \pi t) & -\sin (2 \pi t) \\
\sin (2 \pi t) & \cos (2 \pi t)
\end{array}\right)
$$

Thus, $\hat{\delta}_{1}$ represents $2^{\left[\frac{r}{2}\right]}$ times the generator of $\pi_{1}\left(S O\left(d_{r} m\right)\right)$. Since $r \geq 3$ and $r \equiv 1,7(\bmod 8), 2^{\left[\frac{r}{2}\right]}$ is divisible by 8 and $\hat{\delta}_{1}$ is null homotopic. 
- When $m$ is even and $m \geq 4$, also consider the path

$$
\begin{aligned}
\delta_{2}:[0,1] & \longrightarrow \quad \operatorname{Spin}(m) \times \operatorname{Spin}(r) \\
t & \mapsto\left(\prod_{j=1}^{\frac{m}{2}} \cos (\pi t / 2)+\sin (\pi t / 2) v_{2 j-1} v_{2 j}, \cos (\pi t)+\sin (\pi t) e_{1} e_{2}\right)
\end{aligned}
$$

joining $(1,1)$ to $\left(\operatorname{vol}_{m},-1\right)$ in $\operatorname{Spin}(m) \times \operatorname{Spin}(r)$. It projects to the loop

$\hat{\delta}_{2}:[0,1] \rightarrow \widehat{S O(m)} \widehat{\sin (r)} \subset S O(N)$

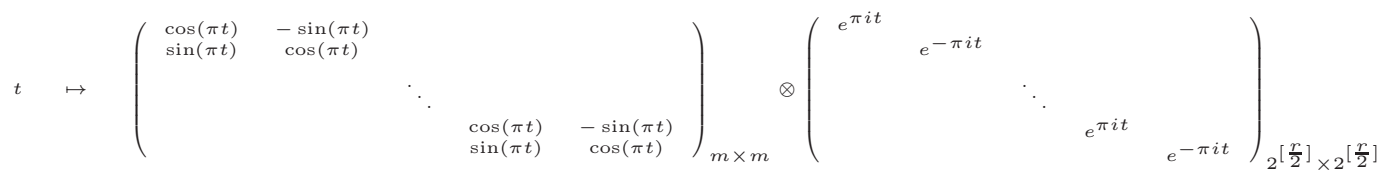

which is similar to

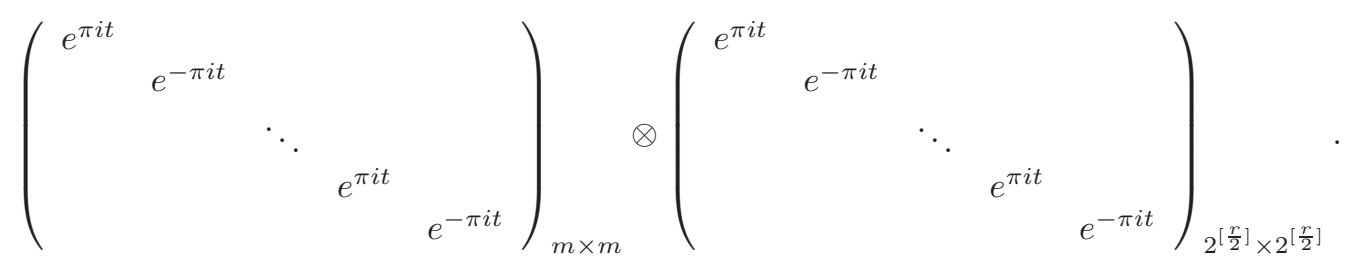

It contains $2^{\left[\frac{r}{2}\right]-1}$ blocks

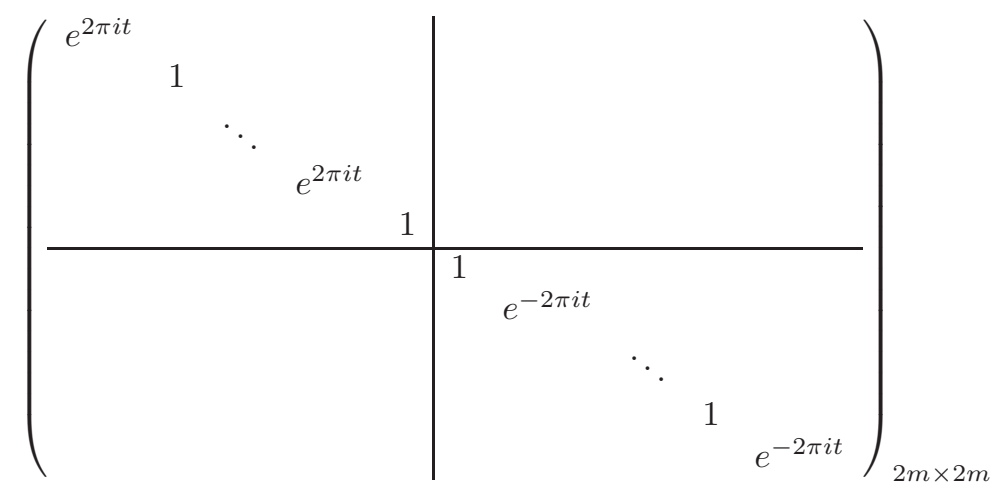

i.e. there are $2^{\left[\frac{r}{2}\right]-1} \frac{m}{2}=2^{\left[\frac{r}{2}\right]-2} m$ copies of the generator of $\pi_{1}\left(S O\left(d_{r} m\right)\right)$. Since $r \geq 3$ and $r \equiv$ $1,7(\bmod 8), 2^{\left[\frac{r}{2}\right]-2}$ is divisible by 2 and $\hat{\delta}_{2}$ is null homotopic.

- For $m=2$, consider the path

$$
\begin{aligned}
\delta_{3}:[0,1] & \longrightarrow \mathbb{R} \times \operatorname{Spin}(r) \\
t & \mapsto\left(\pi t, \cos (\pi t)+\sin (\pi t) e_{1} e_{2}\right)
\end{aligned}
$$

joining $(0,1)$ to $(1,-1)$ in $\mathbb{R} \times \operatorname{Spin}(r)$, which maps to

$$
\begin{aligned}
& \hat{\delta}_{3}:[0,1] \longrightarrow \widehat{\operatorname{SO}(2)} \widehat{\operatorname{Spin}(r)} \subset S O(N)
\end{aligned}
$$

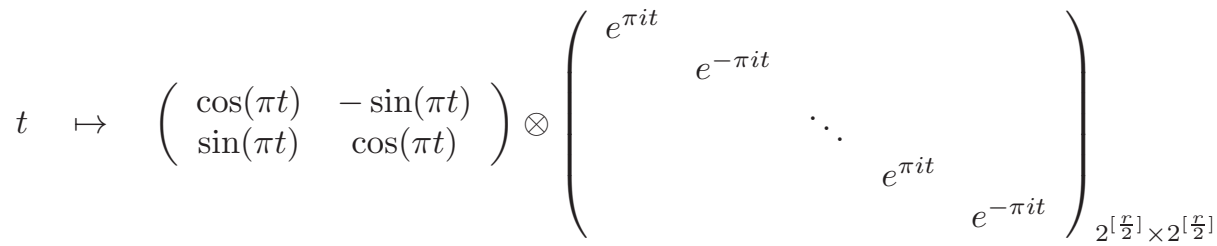

$$
\begin{aligned}
& \sim\left(\begin{array}{cc}
e^{2 \pi i t} & \\
& e^{-2 \pi i t}
\end{array}\right) \otimes \operatorname{Id}_{2^{\left[\frac{r}{2}\right]-1}} \oplus \operatorname{Id}_{2^{\left[\frac{r}{2}\right]-1}} .
\end{aligned}
$$


This loop represents $2^{\left[\frac{r}{2}\right]-1}$ times the generator of $\pi_{1}(S O(N))$, which is null homotopic since $2^{\left[\frac{r}{2}\right]-1}$ is divisible by 4 .

\section{$4.2 r \equiv 0(\bmod 8)$}

Let $r=8 k,\left\{v_{1}, \ldots, v_{m_{1}}\right\}$ and $\left\{v_{1}^{\prime}, \ldots, v_{m_{2}}^{\prime}\right\}$ oriented orthonormal bases of $\mathbb{R}^{m_{1}}$ and $\mathbb{R}^{m_{2}}$ respectively. Recall the fundamental group generators for $m_{1}, m_{2} \geq 3$ :

\begin{tabular}{|c|c|c|c|c|c|c|}
\hline & Cases & $\pi_{1}\left(\widehat{S O\left(m_{1}\right)} \widehat{S O\left(m_{2}\right)} \widehat{\operatorname{Spin}(r)}\right)$ & $(-1,1,1)$ & $(1,-1,1)$ & $\left(\operatorname{vol}_{m_{1}}, 1,-\operatorname{vol}_{r}\right)$ & $\left(1, \operatorname{vol}_{m_{2}}, \operatorname{vol}_{r}\right)$ \\
\hline (a) & $m_{1} \equiv 1(2), m_{2} \equiv 1(2)$ & $\mathbb{Z}_{2} \oplus \mathbb{Z}_{2}$ & $\checkmark$ & $\checkmark$ & & \\
\hline (b) & $m_{1} \equiv 0(4), m_{2} \equiv 1(2)$ & $\mathbb{Z}_{2} \oplus \mathbb{Z}_{2} \oplus \mathbb{Z}_{2}$ & $\checkmark$ & $\checkmark$ & $\checkmark$ & \\
\hline (c) & $m_{1} \equiv 2(4), m_{2} \equiv 1(2)$ & $\mathbb{Z}_{2} \oplus \mathbb{Z}_{4}$ & & $\checkmark$ & $\checkmark$ & \\
\hline (d) & $m_{1} \equiv 1(2), m_{2} \equiv 0(4)$ & $\mathbb{Z}_{2} \oplus \mathbb{Z}_{2} \oplus \mathbb{Z}_{2}$ & $\sqrt{ }$ & $\checkmark$ & & $\checkmark$ \\
\hline (e) & $m_{1} \equiv 1(2), m_{2} \equiv 2(4)$ & $\mathbb{Z}_{2} \oplus \mathbb{Z}_{4}$ & $\checkmark$ & & & $\checkmark$ \\
\hline (f) & $m_{1} \equiv 0(4), m_{2} \equiv 0(4)$ & $\mathbb{Z}_{2} \oplus \mathbb{Z}_{2} \oplus \mathbb{Z}_{2} \oplus \mathbb{Z}_{2}$ & $\checkmark$ & $\checkmark$ & $\checkmark$ & $\checkmark$ \\
\hline (g) & $m_{1} \equiv 0(4), m_{2} \equiv 2(4)$ & $\mathbb{Z}_{2} \oplus \mathbb{Z}_{2} \oplus \mathbb{Z}_{4}$ & $\checkmark$ & & $\checkmark$ & $\checkmark$ \\
\hline (h) & $m_{1} \equiv 2(4), m_{2} \equiv 0(4)$ & $\mathbb{Z}_{2} \oplus \mathbb{Z}_{4} \oplus \mathbb{Z}_{2}$ & & $\checkmark$ & $\checkmark$ & $\checkmark$ \\
\hline (i) & $m_{1} \equiv 2(4), m_{2} \equiv 2(4)$ & $\mathbb{Z}_{4} \oplus \mathbb{Z}_{4}$ & & & $\checkmark$ & $\checkmark$ \\
\hline
\end{tabular}

- For the cases (a), (b), (d), (f) and (g) consider the path

$$
\begin{aligned}
\delta_{1}:[0,1] & \longrightarrow \operatorname{Spin}\left(m_{1}\right) \times \operatorname{Spin}\left(m_{2}\right) \times \operatorname{Spin}(r) \\
t & \mapsto\left(\cos (\pi t)+\sin (\pi t) v_{1} v_{2}, 1,1\right)
\end{aligned}
$$

joining $(1,1,1)$ to $(-1,1,1)$ in $\operatorname{Spin}\left(m_{1}\right) \times \operatorname{Spin}\left(m_{2}\right) \times \operatorname{Spin}(r)$ which projects to the loop

$$
\begin{aligned}
& \left.\hat{\delta}_{1}:[0,1] \longrightarrow\left(\widehat{S O\left(m_{1}\right.}\right) \times \widehat{S O\left(m_{2}\right)}\right) \widehat{\operatorname{Spin}(r)} \subset S O(N) \\
& t \mapsto\left(\begin{array}{ccccc}
\cos (2 \pi t) & -\sin (2 \pi t) & & & \\
\sin (2 \pi t) & \cos (2 \pi t) & & & \\
& & 1 & & \\
& & & \ddots & \\
& & & & 1
\end{array}\right)_{m_{1} \times m_{1}} \otimes \operatorname{Id}_{\Delta_{r}^{+}} \oplus \operatorname{Id}_{m_{2}} \otimes \operatorname{Id}_{\Delta_{r}^{-}}
\end{aligned}
$$

It contains $2^{\frac{r}{2}-1}$ copies of the generator of $\pi_{1}\left(S O\left(d_{r}\left(m_{1}+m_{2}\right)\right)\right)$, which is homotopically trivial since $2^{\frac{r}{2}-1}$ is divisible by 8 .

- For the cases (a), (b), (c), (d), (f) and (h), consider the path

$$
\begin{aligned}
\delta_{2}:[0,1] & \longrightarrow \operatorname{Spin}\left(m_{1}\right) \times \operatorname{Spin}\left(m_{2}\right) \times \operatorname{Spin}(r) \\
t & \mapsto\left(1, \cos (\pi t)+\sin (\pi t) v_{1}^{\prime} v_{2}^{\prime}, 1\right)
\end{aligned}
$$

joining $(1,1,1)$ to $(1,-1,1)$ in $\operatorname{Spin}\left(m_{1}\right) \times \operatorname{Spin}\left(m_{2}\right) \times \operatorname{Spin}(r)$, which projects to the loop

$$
\begin{aligned}
& \left.\hat{\delta}_{2}:[0,1] \longrightarrow\left(\widehat{S O\left(m_{1}\right.}\right) \times \widehat{S O\left(m_{2}\right)}\right) \widehat{\operatorname{Spin}(r)} \subset S O(N)
\end{aligned}
$$

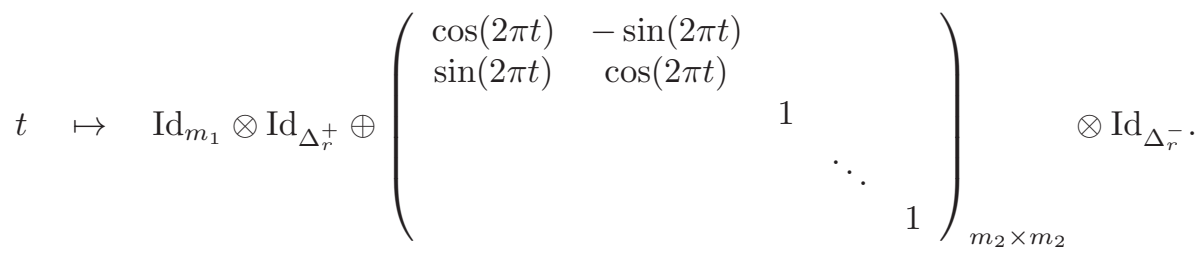

It contains $2^{\frac{r}{2}-1}$ copies of the generator of $\pi_{1}\left(S O\left(d_{r}\left(m_{1}+m_{2}\right)\right)\right)$, and is homotopically trivial since $2^{\frac{r}{2}-1}$ is divisible by 8 . 
- For the cases (b), (c), (f), (g), (h) and (i), consider the path

$$
\begin{aligned}
\delta_{3}:[0,1] & \longrightarrow \operatorname{Spin}\left(m_{1}\right) \times \operatorname{Spin}\left(m_{2}\right) \times \operatorname{Spin}(r) \\
t & \mapsto\left(1, \prod_{j=1}^{\frac{m_{2}}{2}} \cos (\pi t / 2)+\sin (\pi t / 2) v_{2 j-1}^{\prime} v_{2 j}^{\prime}, \prod_{l=1}^{\frac{r}{2}} \cos (\pi t / 2)+\sin (\pi t / 2) e_{2 l-1} e_{2 l}\right)
\end{aligned}
$$

joining $(1,1,1)$ to $\left(1, \operatorname{vol}_{m_{2}}, \operatorname{vol}_{r}\right)$ in $\operatorname{Spin}\left(m_{1}\right) \times \operatorname{Spin}\left(m_{2}\right) \times \operatorname{Spin}(r)$. It projects to the loop

$$
\begin{aligned}
& \hat{\delta}_{3}:[0,1] \longrightarrow\left(\widehat{S O\left(m_{1}\right)} \times \widehat{S O\left(m_{2}\right)}\right) \widehat{\operatorname{Spin}(r)} \subset S O(N) \\
& t \mapsto \quad \operatorname{Id}_{m_{1}} \otimes P^{+}(t) \oplus\left(\begin{array}{ccccc}
\cos (\pi t) & -\sin (\pi t) & & & \\
\sin (\pi t) & \cos (\pi t) & & & \\
& & \ddots & & \\
& & & \cos (\pi t) & -\sin (\pi t) \\
& & & \sin (\pi t) & \cos (\pi t)
\end{array}\right)_{m_{2} \times m_{2}} \otimes P^{-}(t) \\
& \sim \operatorname{Id}_{m_{1}} \otimes P^{+}(t) \oplus\left(\begin{array}{ccccc}
e^{\pi i t} & & & & \\
& e^{-\pi i t} & & & \\
& & \ddots & & \\
& & e^{\pi i t} & \\
& & & & e^{-\pi i t}
\end{array}\right)_{m_{2} \times m_{2}} \otimes P^{-}(t) .
\end{aligned}
$$

where

$$
\begin{aligned}
& P^{+}(t)=\operatorname{diag}(e^{2 \pi(k) i t}, \underbrace{e^{2 \pi(k-1) i t}, \ldots, e^{2 \pi(k-1) i t}}_{\left(\begin{array}{c}
4 k \\
2
\end{array}\right) \text { times }} \cdot \underbrace{e^{2 \pi(k-2) i t}, \ldots, e^{2 \pi(k-2) i t}}_{\left(\begin{array}{c}
4 k \\
4
\end{array}\right) \text { times }}, \ldots, e^{2 \pi(-k) i t}), \\
& P^{-}(t)=\operatorname{diag}(\underbrace{(2 k-1) \pi i t}_{\left(\begin{array}{c}
4 k \\
(2 k-1) \pi i t
\end{array}\right) \text { times }}, \underbrace{e^{(2 k-3) \pi i t}, \ldots, e^{(2 k-3) \pi i t}}_{\left(\begin{array}{c}
4 k \\
3
\end{array}\right) \text { times }}, \ldots, \underbrace{e^{-(2 k-1) \pi i t}, \ldots, e^{-(2 k-1) \pi i t}}_{\left(\begin{array}{c}
4 k \\
4 k-1
\end{array}\right) \text { times }}) .
\end{aligned}
$$

Thus, $\hat{\delta}_{3}$ containts

$$
\begin{aligned}
& \left.m_{1}\left(k+\left(\begin{array}{c}
4 k \\
2
\end{array}\right)(k-1)+\left(\begin{array}{c}
4 k \\
4
\end{array}\right)(k-2)+\cdots+\left(\begin{array}{c}
4 k \\
2 k-2
\end{array}\right)\right)+\frac{m_{2}}{2}\left(\begin{array}{c}
4 k \\
1
\end{array}\right) k+\left(\begin{array}{c}
4 k \\
3
\end{array}\right)(k-1)+\cdots+\left(\begin{array}{c}
4 k \\
4 k-1
\end{array}\right)(-(k-1))\right) \\
= & m_{1} \frac{k(2 k-1)}{8 k-2)}\left(\begin{array}{c}
4 k \\
2 k
\end{array}\right)+2^{4 k-3} m_{2}
\end{aligned}
$$

copies of the generator of $\pi_{1}\left(S O\left(d_{r}\left(m_{1}+m_{2}\right)\right)\right)$. This number of copies is always even except when $k=1$ and $m_{1}$ is odd.

- For the cases (d), (e), (f), (g), (h) and (i), consider the path $\delta_{4}:[0,1] \quad \longrightarrow \quad \operatorname{Spin}\left(m_{1}\right) \times \operatorname{Spin}\left(m_{2}\right) \times \operatorname{Spin}(r)$

$$
t \mapsto\left(\prod_{j=1}^{\frac{m_{1}}{2}}\left(\cos (\pi t / 2)+\sin (\pi t / 2) v_{2 j-1} v_{2 j}, 1,\left(\cos (\pi t / 2)-\sin (\pi t / 2) e_{1} e_{2}\right) \prod_{l=2}^{\frac{r}{2}}\left(\cos (\pi t / 2)+\sin (\pi t / 2) e_{2 l-1} e_{2 l}\right)\right)\right.
$$

joining $(1,1,1)$ to $\left(\operatorname{vol}_{m_{1}}, 1,-\operatorname{vol}_{r}\right)$ in $\operatorname{Spin}\left(m_{1}\right) \times \operatorname{Spin}\left(m_{2}\right) \times \operatorname{Spin}(r)$. It projects to the loop

$$
\hat{\delta}_{4}:[0,1] \longrightarrow\left(\widehat{S O\left(m_{1}\right)} \times S \widehat{S\left(m_{2}\right)}\right) \widehat{\operatorname{Spin}(r)} \subset S O(N)
$$

$$
\begin{aligned}
& t \mapsto\left(\begin{array}{ccccc}
\cos (\pi t) & -\sin (\pi t) & & & \\
\sin (\pi t) & \cos (\pi t) & & & \\
& & \ddots & & \\
& & & \begin{array}{c}
\cos (\pi t) \\
\sin (\pi t)
\end{array} & -\sin (\pi t) \\
& & & \cos (\pi t)
\end{array}\right)_{m_{1} \times m_{1}} \otimes Q^{+}(t) \oplus \operatorname{Id}_{m_{2}} \otimes Q^{-}(t) \\
& \sim\left(\begin{array}{ccccc}
e^{\pi i t} & & & & \\
& e^{-\pi i t} & & & \\
& & \ddots & & \\
& & & e^{\pi i t} & \\
& & & & e^{-\pi i t}
\end{array}\right)_{m_{1} \times m_{1}} \otimes Q^{+}(t) \oplus \operatorname{Id}_{m_{2}} \otimes Q^{-}(t)
\end{aligned}
$$


where

$$
\begin{aligned}
& Q^{+}(t)=\operatorname{diag}(\underbrace{e^{(2 k-3) \pi i t}, \ldots, e^{(2 k-3) \pi i t}}_{e^{(2 k-1) \pi i t}, \ldots, e^{(2 k-1) \pi i t}} \underbrace{e^{(2 k-5) \pi i t}, \ldots, e^{(2 k-5) \pi i t}}_{\left(\begin{array}{c}
4 k \\
1
\end{array}\right) \text { times }}, \cdots, \underbrace{\left.e^{-(2 k-1) \pi i t}, \ldots, e^{-(2 k-1) \pi i t}\right)}_{\left(\begin{array}{c}
4 k \\
5
\end{array}\right) \text { times }}, \\
& Q^{-}(t)=\operatorname{diag}(e^{2 \pi k i t}, \underbrace{e^{2 \pi(k-1) i t}, \ldots, e^{2 \pi(k-1) i t}}_{\left(\begin{array}{c}
4 k \\
4 k
\end{array}\right) \text { times }}, \underbrace{e^{2 \pi(k-2) i t}, \ldots, e^{2 \pi(k-2) i t}}_{\left(\begin{array}{c}
4 k \\
3
\end{array}\right) \text { times }}, \ldots, e^{2 \pi(-k) i t}) .
\end{aligned}
$$

Thus, we have

$$
\begin{aligned}
& \frac{m_{1}}{2}\left[k\left(\begin{array}{c}
4 k \\
1
\end{array}\right)+(k-1)\left(\begin{array}{c}
4 k \\
3
\end{array}\right)+\cdots+(-(k-1))\left(\begin{array}{c}
4 k \\
4 k-1
\end{array}\right)\right]+m_{2}\left[k\left(\begin{array}{c}
4 k \\
0
\end{array}\right)+(k-1)\left(\begin{array}{c}
4 k \\
2
\end{array}\right)+\cdots+1\left(\begin{array}{c}
4 k \\
2 k-2
\end{array}\right)\right] \\
= & 2^{4 k-3} m_{1}+m_{2} \frac{k(2 k-1)}{8 k-2}\left(\begin{array}{c}
4 k \\
2 k
\end{array}\right),
\end{aligned}
$$

which is odd only if $k=1$ and $m_{2} \equiv 1(\bmod 2)$.

The cases in which either $m_{1} \leq 2$ or $m_{2} \leq 2$ are treated similarly.

\section{$4.3 r \equiv 2,6(\bmod 8)$}

Recall

$$
\pi_{1}(\widehat{U(m)} \widehat{\operatorname{Spin}(r)})= \begin{cases}\mathbb{Z}, & \text { if }(m, 4)=1, \\ \mathbb{Z} \oplus \mathbb{Z}_{2}, & \text { if }(m, 4)=2, \\ \mathbb{Z} \oplus \mathbb{Z}_{4}, & \text { if }(m, 4)=4 .\end{cases}
$$

In every case, the fundamental group has generators

$$
\left(\frac{2 \pi}{m}, e^{-\frac{2 \pi i}{m}} \operatorname{Id}_{m}, 1\right), \quad\left(\frac{\pi}{2}, \operatorname{Id}_{m},-\mathrm{vol}\right) .
$$

- Consider the path

$$
\begin{aligned}
\delta_{1}:[0,1] & \longrightarrow \mathbb{R} \times S U(m) \times \operatorname{Spin}(r) \\
t & \mapsto\left(\frac{2 \pi t}{m},\left(\begin{array}{llll}
e^{-\frac{2 \pi i t}{m}} & & \\
& e^{-\frac{2 \pi i t}{m}} & & \\
& & \ddots & \\
& & e^{-\frac{2 \pi i t}{m}} & \\
& & & e^{\frac{2 \pi i(m-1) t}{m}}
\end{array}\right)_{m \times m}, 1\right)
\end{aligned}
$$

joining $\left(0, \operatorname{Id}_{m \times m}, 1\right)$ to $\left(\frac{2 \pi}{m}, e^{-\frac{2 \pi i}{m}} \operatorname{Id}_{m}, 1\right)$ in $\mathbb{R} \times S U(m) \times \operatorname{Spin}(r)$. This path gets mapped to the loop $\hat{\delta}_{1}:[0,1] \longrightarrow \widehat{U(m)} \widehat{\operatorname{Spin}(r)} \subset S O(N)$

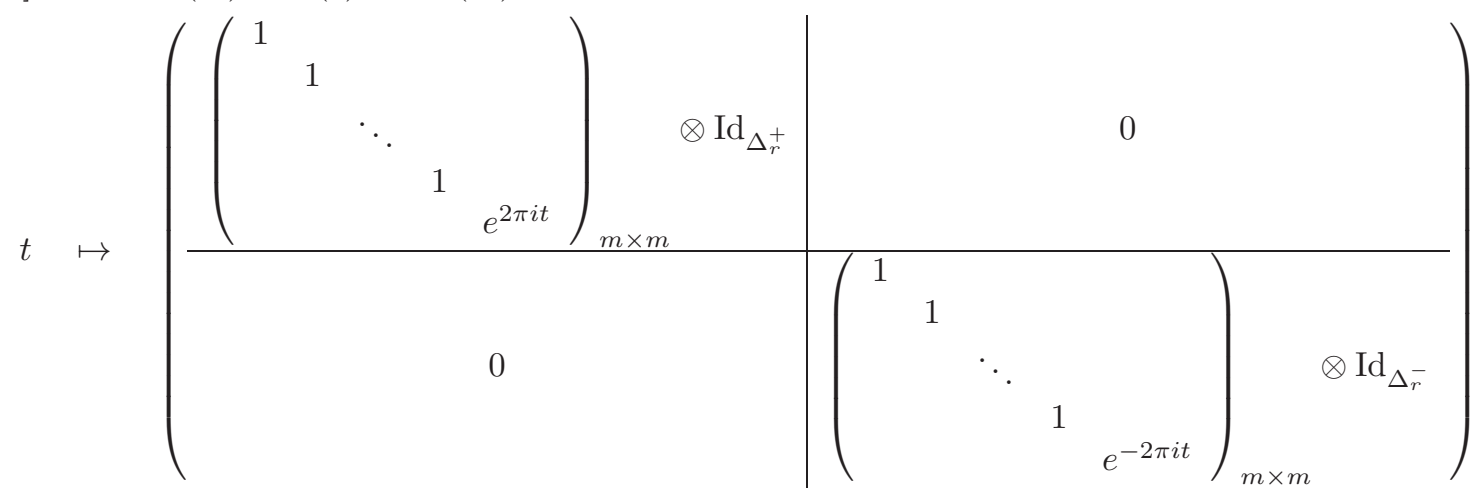


if $r \equiv 2(\bmod 8)$, and to

$\hat{\delta}_{1}:[0,1] \longrightarrow \widehat{U(m)} \widehat{\operatorname{Spin}(r)} \subset S O(N)$

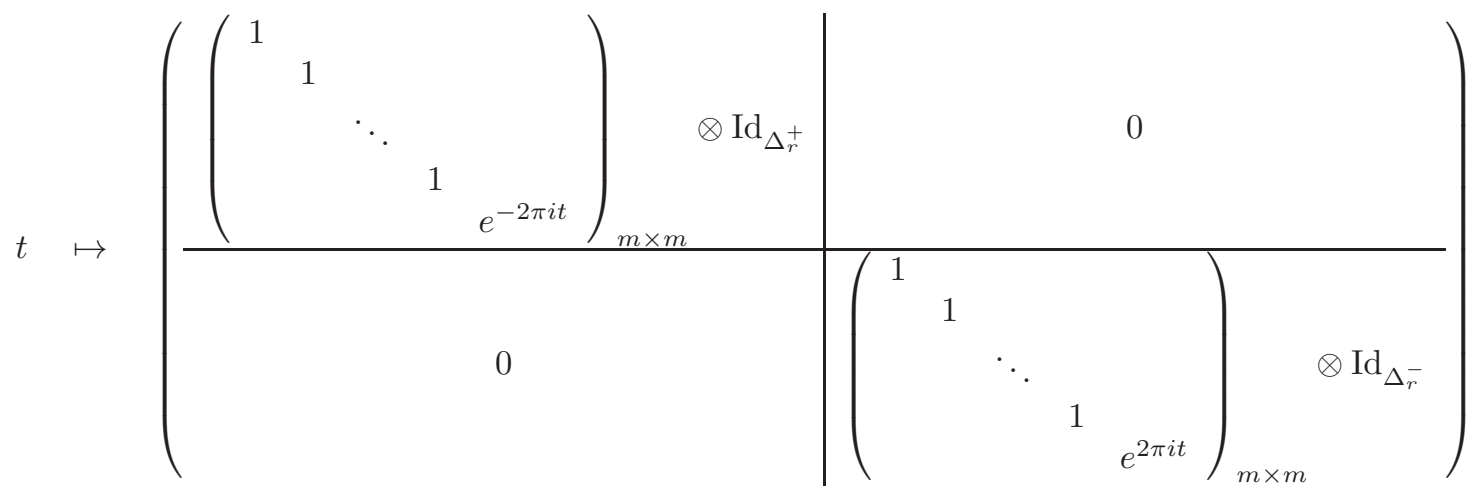

if $r \equiv 6(\bmod 8)$. Since $\operatorname{dim}\left(\Delta_{r}^{ \pm}\right)=2^{\frac{r}{2}-1}$, we have $2^{\frac{r}{2}-1}$ blocks of the form

$$
\left(\begin{array}{cc}
e^{2 \pi i t} & \\
& e^{-2 \pi i t}
\end{array}\right) \sim\left(\begin{array}{cc}
\cos (2 \pi t) & -\sin (2 \pi t) \\
\sin (2 \pi t) & \cos (2 \pi t)
\end{array}\right)
$$

i.e. $2^{\frac{r}{2}-1}$ times the generator of $\pi_{1}\left(S O\left(d_{r} m\right)\right)$. Since $r \geq 6,2^{\frac{r}{2}-1}$ is divisible by 4 . Hence, $\hat{\delta}_{1}$ is null homotopic.

- Consider the path

$$
\begin{aligned}
\delta_{2}:[0,1] & \longrightarrow \mathbb{R} \times S U(m) \times \operatorname{Spin}(r) \\
t & \mapsto\left(\frac{\pi t}{2}, \operatorname{Id}_{m}, \prod_{j=1}^{\frac{r}{2}}\left(\cos (\pi t / 2)-\sin (\pi t / 2) e_{2 j-1} e_{2 j}\right)\right)
\end{aligned}
$$

joining $\left(0, \operatorname{Id}_{m \times m}, 1\right)$ to $\left(\frac{\pi}{2}, \operatorname{Id}_{m},-\mathrm{vol}\right)$ in $\mathbb{R} \times S U(m) \times \operatorname{Spin}(r)$. This path gets mapped to the loop

$$
\begin{aligned}
\hat{\delta}_{2}:[0,1] & \longrightarrow \widehat{U(m)} \widehat{\operatorname{Spin}(r)} \subset S O(N) \\
t & \mapsto\left(\begin{array}{c|c}
e^{\frac{\pi i t}{2}} \operatorname{Id}_{m} \otimes P^{+}(t) & 0 \\
\hline 0 & e^{-\frac{\pi i t}{2}} \operatorname{Id}_{m} \otimes P^{-}(t)
\end{array}\right)
\end{aligned}
$$

if $r \equiv 2(\bmod 8)$, and

$$
\begin{aligned}
\hat{\delta}_{2}:[0,1] & \longrightarrow \widehat{U(m)} \widehat{\operatorname{Spin}(r)} \subset S O(N) \\
t & \mapsto\left(\begin{array}{c|c}
e^{-\frac{\pi i t}{2}} \operatorname{Id}_{m} \otimes P^{+}(t) & 0 \\
\hline 0 & e^{\frac{\pi i t}{2}} \operatorname{Id}_{m} \otimes P^{-}(t)
\end{array}\right)
\end{aligned}
$$

if $r \equiv 6(\bmod 8)$, where

$$
\begin{aligned}
& P^{+}(t)=\operatorname{diag}(e^{-\frac{r}{2} \frac{\pi i t}{2}}, \underbrace{e^{-\left(\frac{r}{2}-4\right) \frac{\pi i t}{2}}, \ldots, e^{-\left(\frac{r}{2}-4\right) \frac{\pi i t}{2}}}_{\left(\begin{array}{c}
r / 2 \\
2
\end{array}\right) \text { times }}, \underbrace{e^{-\left(\frac{r}{2}-8\right) \frac{\pi i t}{2}}, \ldots, e^{-\left(\frac{r}{2}-8\right) \frac{\pi i t}{2}}}_{\left(\begin{array}{c}
r / 2 \\
4
\end{array}\right) \text { times }}, \ldots), \\
& P^{-}(t)=\operatorname{diag}(\underbrace{e^{-\left(\frac{r}{2}-2\right) \frac{\pi i t}{2}}, \ldots, e^{-\left(\frac{r}{2}-2\right) \frac{\pi i t}{2}}}_{\left(\begin{array}{c}
r / 2 \\
1
\end{array}\right) \text { times }}, \underbrace{e^{-\left(\frac{r}{2}-6\right) \frac{\pi i t}{2}}, \ldots, e^{-\left(\frac{r}{2}-6\right) \frac{\pi i t}{2}}}_{\left(\begin{array}{c}
r / 2 \\
3
\end{array}\right) \text { times }}, \underbrace{e^{-\left(\frac{r}{2}-10\right) \frac{\pi i t}{2}}, \ldots, e^{-\left(\frac{r}{2}-10\right) \frac{\pi i t}{2}}}_{\left(\begin{array}{c}
r / 2 \\
5
\end{array}\right) \text { times }}, \ldots) .
\end{aligned}
$$

- If $r \equiv 2(\bmod 8), r=8 k+2$ with $k \geq 1$. Then $\frac{r}{2}=4 k+1$. We have

$$
m\left[k\left(\begin{array}{c}
4 k+1 \\
0
\end{array}\right)+(k-1)\left(\begin{array}{c}
4 k+1 \\
2
\end{array}\right)+(k-2)\left(\begin{array}{c}
4 k+1 \\
4
\end{array}\right)+\cdots+(-k+1)\left(\begin{array}{c}
4 k+1 \\
4 k-2
\end{array}\right)+(-k)\left(\begin{array}{c}
4 k+1 \\
4 k
\end{array}\right)\right]=-m 2^{4 k-2} .
$$

copies of the generator of $\pi_{1}\left(S O\left(d_{r} m\right)\right)$, which is even and $\hat{\delta}_{1}$ is null homotopic. 
- If $r \equiv 6(\bmod 8), r=8 k+6$ with $k \geq 1$. Then $\frac{r}{2}=4 k+3$. Thus, we have

$$
m\left[(k+1)\left(\begin{array}{c}
4 k+3 \\
0
\end{array}\right)+(k)\left(\begin{array}{c}
4 k+3 \\
2
\end{array}\right)+(k-1)\left(\begin{array}{c}
4 k+3 \\
4
\end{array}\right)+\cdots+(-k+1)\left(\begin{array}{c}
4 k+3 \\
4 k
\end{array}\right)+(-k)\left(\begin{array}{c}
4 k+3 \\
4 k+2
\end{array}\right)\right]=m 2^{4 k}
$$

copies of the generator of $\pi_{1}\left(S O\left(d_{r} m\right)\right)$. If $k \geq 1$, this number is always even and $\hat{\delta}_{2}$ is null homotopic. On the other hand, if $r=6(k=0)$, then the parity of the number depends on $m$.

\section{$4.4 r \equiv 3,5(\bmod 8)$}

Recall

$$
\left.\pi_{1}(\widehat{\operatorname{Sp}(m)} \widehat{\operatorname{Spin}(r})\right)=\mathbb{Z}_{2}=\left\langle\left(-\operatorname{Id}_{2 m},-1\right)\right\rangle
$$

Thus, consider the path

$$
\begin{aligned}
& \delta:[0,1] \quad \longrightarrow \quad S p(m) \times \operatorname{Spin}(r)
\end{aligned}
$$

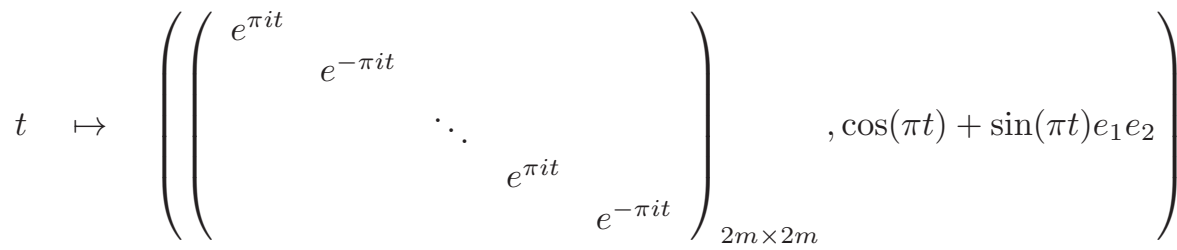

joining $\left(\operatorname{Id}_{2 m}, 1\right)$ to $\left(-\mathrm{Id}_{2 m},-1\right)$ in $\operatorname{Sp}(m) \times \operatorname{Spin}(r)$. It projects to the loop in $\left.\widehat{\operatorname{Sp}(m)} \widehat{\operatorname{Spin}(r}\right) \subset \operatorname{SO}\left(d_{r} m\right)$

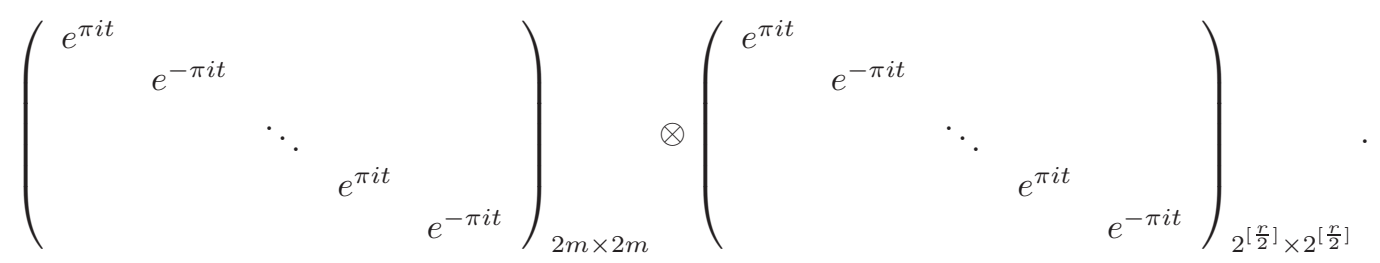

It has $2^{\left[\frac{r}{2}\right]-1} m$ blocks of the form

$$
\left(\begin{array}{ll}
e^{2 \pi i t} & \\
& e^{-2 \pi i t}
\end{array}\right) \sim\left(\begin{array}{ll}
\cos (2 \pi t) & -\sin (2 \pi t) \\
\sin (2 \pi t) & \cos (2 \pi t)
\end{array}\right),
$$

i.e. $2^{\left[\frac{r}{2}\right]-1} m$ times the generator of $\pi_{1}\left(S O\left(d_{r} m\right)\right)=\mathbb{Z}_{2}$. Note that $2^{\left[\frac{r}{2}\right]-1} m$ is divisible by 2 if $r>3$.

\section{5 $r \equiv 4(\bmod 8)$}

Let $r=8 k+4$. Recall

$$
\begin{aligned}
& \left.\left.\pi_{1}\left(\left(\widehat{\operatorname{Sp(m_{1}}}\right) \times \widehat{\operatorname{Sp(m_{2}}}\right)\right) \widehat{\operatorname{Spin}(r)}\right)=\left\langle\left(-\mathrm{Id}_{2 m_{1}},-\mathrm{Id}_{2 m_{2}},-1\right)\right\rangle \times\left\langle\left(\operatorname{Id}_{2 m_{1}},-\operatorname{Id}_{2 m_{2}}, \operatorname{vol}_{r}\right)\right\rangle \subset \operatorname{Sp}\left(m_{1}\right) \times \operatorname{Sp}\left(m_{2}\right) \times \operatorname{Spin}(r) . \\
& \pi_{1}\left(\widehat{\left.\operatorname{Sp(m_{1}}\right) \operatorname{Spin}(r)}-\right) \cong \begin{cases}\left\langle\left(-\mathrm{Id}_{2 m_{1}},-\operatorname{vol}_{r}\right),\left(-\mathrm{Id}_{2 m_{1}},-1\right)\right\rangle \subset \operatorname{Sp}\left(m_{1}\right) \times \operatorname{Spin}(r), & \text { if } m_{1}>0, m_{2}=0, r>4, \\
\left\langle\left(-\mathrm{Id}_{2 m_{1}},-\operatorname{vol}_{4}\right)\right\rangle \subset \operatorname{Sp}\left(m_{1}\right) \times \operatorname{Spin}(4), & \text { if } m_{1}>0, m_{2}=0, r=4,\end{cases} \\
& \pi_{1}\left(\widehat{\left.\operatorname{Sp(m_{2}}\right)} \widehat{\operatorname{Spin}(r)}+\right) \cong\left\{\begin{array}{l}
\left\langle\left(-\mathrm{Id}_{2 m_{2}}, \operatorname{vol}_{r}\right),\left(-\mathrm{Id}_{2 m_{2}},-1\right)\right\rangle \subset \operatorname{Sp}\left(m_{2}\right) \times \operatorname{Spin}(r), \quad \text { if } m_{1}=0, m_{2}>0, r>4, \\
\left\langle\left(-\mathrm{Id}_{2} m_{2}, \mathrm{vol}_{4}\right)\right\rangle \subset \operatorname{Sp}\left(m_{2}\right)
\end{array}\right.
\end{aligned}
$$

- Consider the path

$$
\begin{aligned}
& \delta_{1}:[0,1] \quad \longrightarrow \quad S p\left(m_{1}\right) \times S p\left(m_{2}\right) \times \operatorname{Spin}(r)
\end{aligned}
$$

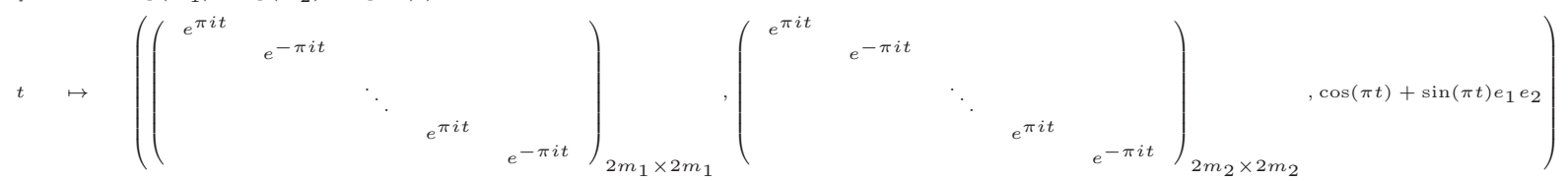


joining $\left(\operatorname{Id}_{2 m_{1}}, \operatorname{Id}_{2 m_{2}}, 1\right)$ and $\left(-\operatorname{Id}_{2 m_{1}},-\mathrm{Id}_{2 m_{2}},-1\right)$ in $S p\left(m_{1}\right) \times S p\left(m_{2}\right) \times \operatorname{Spin}(r)$. It maps to the loop

$$
\begin{aligned}
\hat{\delta}_{1}:[0,1] \longrightarrow & \left.\left.\left.\left(\widehat{\operatorname{Sp}\left(m_{1}\right.}\right) \times \widehat{\operatorname{Sp}\left(m_{2}\right.}\right)\right) \widehat{\operatorname{Spin}(r}\right) \subset S O(N) \\
t \mapsto \quad & \operatorname{diag}\left(e^{\pi i t}, e^{-\pi i t}, \ldots, e^{\pi i t}, e^{-\pi i t}\right)_{2 m_{1} \times 2 m_{1}} \otimes \operatorname{diag}\left(e^{\pi i t}, e^{-\pi i t}, \ldots, e^{\pi i t}, e^{-\pi i t}\right)_{2^{\frac{r}{2}-1} \times 2^{\frac{r}{2}-1}} \\
& \oplus \operatorname{diag}\left(e^{\pi i t}, e^{-\pi i t}, \ldots, e^{\pi i t}, e^{-\pi i t}\right)_{2 m_{2} \times 2 m_{2}} \otimes \operatorname{diag}\left(e^{\pi i t}, e^{-\pi i t}, \ldots, e^{\pi i t}, e^{-\pi i t}\right)_{2^{\frac{r}{2}-1} \times 2^{\frac{r}{2}-1}} \\
\sim & 2^{\frac{r}{2}-2} \operatorname{diag}\left(e^{2 \pi i t}, e^{-2 \pi i t}, \ldots, e^{2 \pi i t}, e^{-2 \pi i t}\right)_{2 m_{1} \times 2 m_{1}} \oplus 2^{\frac{r}{2}-2} \operatorname{Id}_{2 m_{1}} \\
& \oplus 2^{\frac{r}{2}-2} \operatorname{diag}\left(e^{2 \pi i t}, e^{-2 \pi i t}, \ldots, e^{2 \pi i t}, e^{-2 \pi i t}\right)_{2 m_{2} \times 2 m_{2}} \oplus 2^{\frac{r}{2}-2} \operatorname{Id}_{2 m_{2}}
\end{aligned}
$$

which is homotopically equivalent to $\left(m_{1}+m_{2}\right) 2^{\frac{r}{2}-2}$ times the generator of $\pi_{1}\left(S O\left(d_{r}\left(m_{1}+m_{2}\right)\right)\right)$. Hence, $\hat{\delta}_{1}$ is null homotopic if either $r \neq 4$ or $r=4$ and $m_{1}+m_{2} \equiv 0(\bmod 2)$.

- Consider the path

$$
\begin{aligned}
& \delta_{2}:[0,1] \quad \longrightarrow \quad S p\left(m_{1}\right) \times S p\left(m_{2}\right) \times \operatorname{Spin}(r)
\end{aligned}
$$

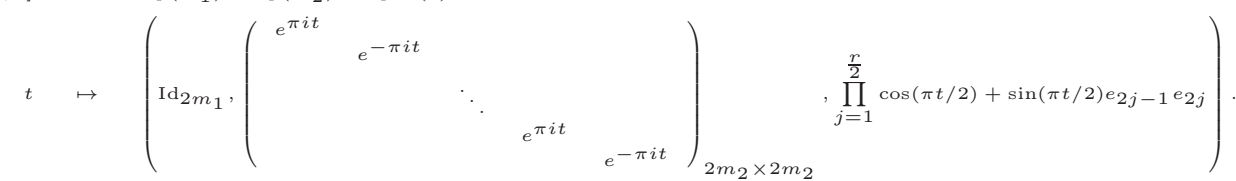

joining $\left(\operatorname{Id}_{2 m_{1}}, \operatorname{Id}_{2 m_{2}}, 1\right)$ to $\left(\operatorname{Id}_{2 m_{1}},-\mathrm{Id}_{2 m_{2}}, \operatorname{vol}_{r}\right)$. It maps to the loop

$$
\begin{aligned}
\hat{\delta}_{2} & \left.\longrightarrow\left(\widehat{\operatorname{Sp(m_{1}}}\right) \times \widehat{\operatorname{Sp(m_{2}}}\right) \hat{\operatorname{Spin}(r)} \subset S O(N) \\
t & \mapsto \quad \operatorname{Id}_{2 m_{1}} \otimes P^{-}(t) \oplus \operatorname{diag}\left(e^{\pi i t}, e^{-\pi i t}, \ldots, e^{\pi i t}, e^{-\pi i t}\right)_{2 m_{2} \times 2 m_{2}} \otimes P^{+}(t),
\end{aligned}
$$

where

$$
\begin{aligned}
& P^{+}(t)=\operatorname{diag}(e^{(2 k+1) \pi i t}, \underbrace{e^{(2 k-1) \pi i t}, \ldots, e^{(2 k-1) \pi i t}}_{\left(\begin{array}{c}
4 k+2 \\
2
\end{array}\right) \text { times }}, \underbrace{e^{(2 k-3) \pi i t}, \ldots, e^{(2 k-3) \pi i t}}_{\left(\begin{array}{c}
4 k+2 \\
4
\end{array}\right) \text { times }}, \ldots, e^{-(2 k+1) \pi i t}), \\
& P^{-}(t)=\operatorname{diag}(\underbrace{e^{2 \pi k i t}, \ldots, e^{2 \pi k i t}}_{\left(\begin{array}{c}
4 k+2 \\
1
\end{array}\right) \text { times }}, \underbrace{e^{2 \pi(k-1) i t}, \ldots, e^{2 \pi(k-1) i t}}_{\left(\begin{array}{c}
4 k+2 \\
3
\end{array}\right) \text { times }}, \ldots, \underbrace{e^{2 \pi(-k) i t}, \ldots, e^{2 \pi(-k) i t}}_{\left(\begin{array}{c}
4 k+2 \\
4 k+1
\end{array}\right) \text { times }}) .
\end{aligned}
$$

This loop is homotopically equivalent $(\bmod 2)$ to

$$
\begin{aligned}
& m_{2}\left(\left(\begin{array}{c}
4 k+2 \\
0
\end{array}\right)(k+1)+\left(\begin{array}{c}
4 k+2 \\
2
\end{array}\right) k+\left(\begin{array}{c}
4 k+2 \\
4
\end{array}\right)(k-1)+\cdots+\left(\begin{array}{c}
4 k+2 \\
4 k
\end{array}\right)(-(k-1))+\left(\begin{array}{c}
4 k+2 \\
4 k+2
\end{array}\right)(-k)\right)+2 m_{1}\left(\left(\begin{array}{c}
4 k+2 \\
1
\end{array}\right) k+\left(\begin{array}{c}
4 k+2 \\
3
\end{array}\right)(k-1)+\cdots+\left(\begin{array}{c}
4 k+2 \\
2 k-1
\end{array}\right)\right) \\
= & m_{2} 16^{k}+2 m_{1} \frac{k(2 k+1)}{8 k+2}\left(\begin{array}{c}
4 k+2 \\
2 k+1
\end{array}\right)
\end{aligned}
$$

times the generator of $\pi_{1}(S O(N))$, which is trivial if $k \geq 1$.

- Consider the path

$$
\begin{aligned}
& \delta_{3}:[0,1] \quad \longrightarrow \quad \operatorname{Sp}\left(m_{1}\right) \times \operatorname{Spin}(r) \\
& t \mapsto\left(\left(\begin{array}{ccccc}
e^{\pi i t} & & & & \\
& e^{-\pi i t} & & & \\
& & \ddots & & \\
& & & e^{\pi i t} & \\
& & & & e^{-\pi i t}
\end{array}\right)_{2 m_{1} \times 2 m_{1}}, \cos (\pi t)+\sin (\pi t) e_{1} e_{2}\right)
\end{aligned}
$$

joining $\left(\mathrm{Id}_{2 m_{1}}, 1\right)$ and $\left(-\mathrm{Id}_{2 m_{1}},-1\right)$ in $S p\left(m_{1}\right) \times \operatorname{Spin}(r)$. It maps to the loop

$$
\begin{aligned}
\hat{\delta}_{3}:[0,1] & \left.\longrightarrow \widehat{\operatorname{Sp}\left(m_{1}\right.}\right) \widehat{\operatorname{Spin}(r)}-\subset S O(N) \\
t & \mapsto \operatorname{diag}\left(e^{\pi i t}, e^{-\pi i t}, \ldots, e^{\pi i t}, e^{-\pi i t}\right)_{2 m_{1} \times 2 m_{1}} \otimes \operatorname{diag}\left(e^{\pi i t}, e^{-\pi i t}, \ldots, e^{\pi i t}, e^{-\pi i t}\right)_{2^{\frac{r}{2}-1} \times 2^{\frac{r}{2}-1}} \\
& \sim 2^{\frac{r}{2}-2} \operatorname{diag}\left(e^{2 \pi i t}, e^{-2 \pi i t}, \ldots, e^{2 \pi i t}, e^{-2 \pi i t}\right)_{2 m_{1} \times 2 m_{1}} \oplus 2^{\frac{r}{2}-2} \operatorname{Id}_{2 m_{1}}
\end{aligned}
$$

which is homotopically equivalent to $m_{1} 2^{\frac{r}{2}-2}$ times the generator of $\pi_{1}\left(S O\left(d_{r}\left(m_{1}\right)\right)\right)$. Hence, $\hat{\delta}_{3}$ is null homotopic since $r \neq 4$. 
- Consider the path

$$
\begin{aligned}
& \delta_{4}:[0,1] \quad \longrightarrow \quad \operatorname{Sp}\left(m_{2}\right) \times \operatorname{Spin}(r) \\
& t \mapsto\left(\left(\begin{array}{ccccc}
e^{\pi i t} & & & & \\
& e^{-\pi i t} & & & \\
& & \ddots & & \\
& & & e^{\pi i t} & \\
& & & & e^{-\pi i t}
\end{array}\right)_{2 m_{2} \times 2 m_{2}}, \cos (\pi t)+\sin (\pi t) e_{1} e_{2}\right)
\end{aligned}
$$

joining $\left(\operatorname{Id}_{2 m_{2}}, 1\right)$ and $\left(-\operatorname{Id}_{2 m_{2}},-1\right)$ in $\operatorname{Sp}\left(m_{2}\right) \times \operatorname{Spin}(r)$. It maps to the loop

$$
\begin{aligned}
\hat{\delta}_{4}:[0,1] & \left.\longrightarrow \widehat{\operatorname{Sp(m_{2}}}\right) \widehat{\operatorname{Spin}(r)}+\subset S O(N) \\
t & \mapsto \operatorname{diag}\left(e^{\pi i t}, e^{-\pi i t}, \ldots, e^{\pi i t}, e^{-\pi i t}\right)_{2 m_{2} \times 2 m_{2}} \otimes \operatorname{diag}\left(e^{\pi i t}, e^{-\pi i t}, \ldots, e^{\pi i t}, e^{-\pi i t}\right)_{2^{\frac{r}{2}-1} \times 2^{\frac{r}{2}-1}} \\
& \sim 2^{\frac{r}{2}-2} \operatorname{diag}\left(e^{2 \pi i t}, e^{-2 \pi i t}, \ldots, e^{2 \pi i t}, e^{-2 \pi i t}\right)_{2 m_{2} \times 2 m_{2}} \oplus 2^{\frac{r}{2}-2} \operatorname{Id}_{2 m_{2}}
\end{aligned}
$$

which is homotopically equivalent to $m_{2} 2^{\frac{r}{2}-2}$ times the generator of $\pi_{1}(S O(N))$. Hence, $\hat{\delta}_{4}$ is null homotopic since $r \neq 4$.

- Consider the path

$$
\begin{aligned}
& \delta_{5}:[0,1] \quad \longrightarrow \quad S p\left(m_{2}\right) \times \operatorname{Spin}(r) \\
& t \mapsto\left(\left(\begin{array}{ccccc}
e^{\pi i t} & \mapsto i t & & & \\
& e^{-\pi i t} & \ddots & & \\
& & & e^{\pi i t} & \\
& & & & e^{-\pi i t}
\end{array}\right)_{2 m_{2} \times 2 m_{2}}, \prod_{j=1}^{\frac{r}{2}} \cos (\pi t / 2)+\sin (\pi t / 2) e_{2 j-1} e_{2 j}\right) .
\end{aligned}
$$

joining $\left(\operatorname{Id}_{2 m_{2}}, 1\right)$ to $\left(-\mathrm{Id}_{2 m_{2}}, \mathrm{vol}_{r}\right)$. It maps to the loop

$$
\begin{aligned}
\hat{\delta}_{5} & \longrightarrow \widehat{\operatorname{Sp}\left(m_{2}\right)} \widehat{\operatorname{Spin(r)}}+\subset S O(N) \\
t & \mapsto \operatorname{diag}\left(e^{\pi i t}, e^{-\pi i t}, \ldots, e^{\pi i t}, e^{-\pi i t}\right)_{2 m_{2} \times 2 m_{2}} \otimes P^{+}(t),
\end{aligned}
$$

where

$$
P^{+}(t)=\operatorname{diag}(e^{(2 k+1) \pi i t}, \underbrace{e^{(2 k-1) \pi i t}, \ldots, e^{(2 k-1) \pi i t}}_{\left(\begin{array}{c}
4 k+2 \\
2
\end{array}\right) \text { times }}, \underbrace{e^{(2 k-3) \pi i t}, \ldots, e^{(2 k-3) \pi i t}}_{\left(\begin{array}{c}
4 k+2 \\
4
\end{array}\right) \text { times }}, \ldots, e^{-(2 k+1) \pi i t}),
$$

This loop is homotopically equivalent $(\bmod 2)$ to

$$
m_{2}\left(\left(\begin{array}{c}
4 k+2 \\
0
\end{array}\right)(k+1)+\left(\begin{array}{c}
4 k+2 \\
2
\end{array}\right) k+\left(\begin{array}{c}
4 k+2 \\
4
\end{array}\right)(k-1)+\cdots+\left(\begin{array}{c}
4 k+2 \\
4 k
\end{array}\right)(-(k-1))+\left(\begin{array}{c}
4 k+2 \\
4 k+2
\end{array}\right)(-k)\right)=m_{2} 16^{k}
$$

times the generator of $\pi_{1}(S O(N))$, which is trivial if $k \geq 1$.

- Consider the path

$$
\begin{aligned}
& \delta_{6}:[0,1] \quad \longrightarrow \quad S p\left(m_{1}\right) \times \operatorname{Spin}(r)
\end{aligned}
$$

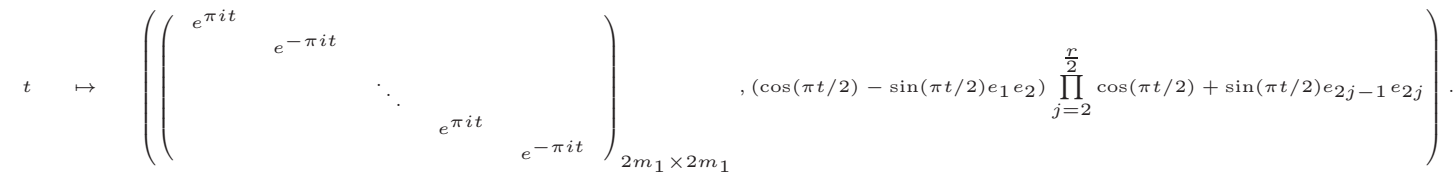

joining $\left(\operatorname{Id}_{2 m_{1}}, 1\right)$ to $\left(-\mathrm{Id}_{2 m_{1}},-\mathrm{vol}_{r}\right)$. It maps to the loop

$$
\begin{aligned}
\hat{\delta}_{6} & \longrightarrow \widehat{\operatorname{Sp(m_{2})}} \widehat{\operatorname{Spin(r)}}-\subset S O(N) \\
t & \mapsto \operatorname{diag}\left(e^{\pi i t}, e^{-\pi i t}, \ldots, e^{\pi i t}, e^{-\pi i t}\right)_{2 m_{2} \times 2 m_{2}} \otimes Q^{-}(t),
\end{aligned}
$$

where

$$
Q^{-}(t)=\operatorname{diag}(e^{(2 k+1) \pi i t}, \underbrace{e^{(2 k-1) \pi i t}, \ldots, e^{(2 k-1) \pi i t}}_{\left(\begin{array}{c}
4 k+2 \\
2
\end{array}\right) \text { times }}, \underbrace{e^{(2 k-3) \pi i t}, \ldots, e^{(2 k-3) \pi i t}}_{\left(\begin{array}{c}
4 k+2 \\
4
\end{array}\right) \text { times }}, \ldots, e^{-(2 k+1) \pi i t}),
$$


This loop is homotopically equivalent $(\bmod 2)$ to

$$
m_{1}\left(\left(\begin{array}{c}
4 k+2 \\
0
\end{array}\right)(k+1)+\left(\begin{array}{c}
4 k+2 \\
2
\end{array}\right) k+\left(\begin{array}{c}
4 k+2 \\
4
\end{array}\right)(k-1)+\cdots+\left(\begin{array}{c}
4 k+2 \\
4 k
\end{array}\right)(-(k-1))+\left(\begin{array}{c}
4 k+2 \\
4 k+2
\end{array}\right)(-k)\right)=m_{1} 16^{k}
$$

times the generator of $\pi_{1}(S O(N))$, which is trivial if $k \geq 1$.

\section{References}

[1] Arizmendi, G.; Hadfield, C.: Twistor Spaces of Riemannian Manifolds with Even Clifford Structures arXiv:1602.04159

[2] Arizmendi, G.; Herrera, R.: Centralizers of spin subalgebras. J. Geom. Phys. 97 (2015) 77-92

[3] Arizmendi, G.; Herrera, R.; Santana, N.: Almost even-Clifford hermitian manifolds with a large automorphism group Proc. Amer. Math. Soc. (2016) dx.doi.org/10.1090/proc/13071

[4] Barberis, M. L.; Dotti, I. G.; Miatello, R. J.: On certain locally homogeneous Clifford manifolds. Annals of Global Analysis and Geometry August 1995, 13, Issue 3, 289-301

[5] Dearricott, O.: Quaternion-Sasakian Manifolds. International Mathematics Research Notices, Vol. 2015, No. 18, pp. 8799-8829.

[6] Friedrich, T.: Dirac operators in Riemannian geometry. Translated from the 1997 German original by Andreas Nestke. Graduate Studies in Mathematics, 25. American Mathematical Society, Providence, RI, 2000. xvi+195 pp. ISBN: 0-8218-2055-9

[7] Joyce, D.: Manifolds with many complex structures. Quart. J. Math. Oxford Ser. (2) 46 (1995), no. 182, $169-184$.

[8] Lawson, H. B., Jr.; Michelsohn, M.-L.: Spin geometry. Princeton Mathematical Series, 38. Princeton University Press, Princeton, NJ, 1989. xii+427 pp. ISBN: 0-691-08542-0

[9] Moroianu, A.; Pilca, M.: Higher Rank Homogeneous Clifford Structures. J. London Math. Soc. 87, (2013), 384-400

[10] A. Moroianu, U. Semmelmann, Clifford structures on Riemannian manifolds. Adv. Math. 228 (2) (2011) $940-967$

[11] Nikolayevsky, Y.: Osserman manifolds and Clifford structures. Houston J. Math. 29 (2003), no. 1, 59-75.

[12] Parton, M.; Piccinni, P.: The even Clifford structure of the fourth Severi variety. Complex Manifolds 2015; 2, 89-104 